Supporting Information for:

\title{
Thiol-Ene Elastomers Derived from Biobased Phenolic Acids with Varying Functionality
}

Guozhen Yang, ${ }^{1}$ Samantha L. Kristufek, ${ }^{2}$ Lauren A. Link, ${ }^{2}$ Karen L. Wooley, ${ }^{2}$ and Megan L. Robertson ${ }^{1, *}$

${ }^{1}$ Department of Chemical and Biomolecular Engineering, University of Houston, Houston, TX, 77204-4004, United States

${ }^{2}$ Department of Chemistry, Department of Chemical Engineering, Department of Materials Science \& Engineering, Texas A\&M University, College Station, Texas 77842-3012, United States

*Corresponding author 4800 Calhoun Road S222 Engineering Building 1

University of Houston Houston, TX 77204-4004

mlrobertson@uh.edu 713-743-2748 


\section{Section 1: NMR Characterization of 3-Hydroxybenzoic Acid}

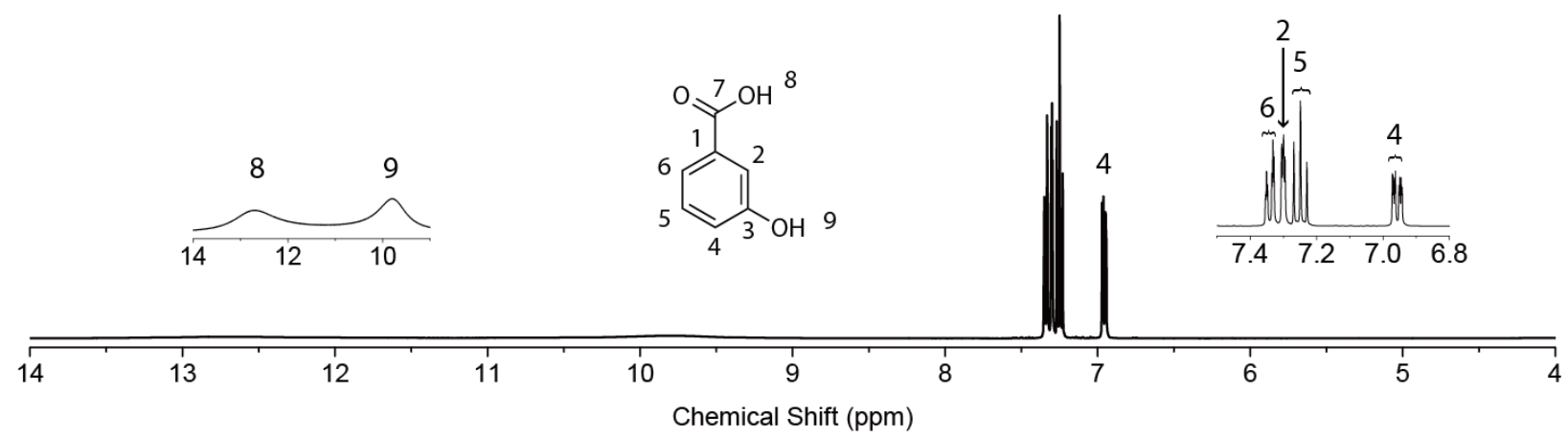

Figure S1a: Chemical structure of and ${ }^{1} \mathrm{H}$ NMR data obtained from 3-hydroxybenzoic acid (referred to as "3HBA" in main text). ${ }^{1} \mathrm{H}$ NMR (400 MHz, DMSO-d 6 , ppm): $\delta 12.74$ (broad s, 1H), 9.75 (broad s, 1H), 7.33 (d, J=7.56 Hz, 1H), $7.29(\mathrm{~s}, 1 \mathrm{H}), 7.24$ (t, $J=7.56 \mathrm{~Hz}, 1 \mathrm{H}), 6.95$ (d, $J=7.56 \mathrm{~Hz}, 1 \mathrm{H})$.

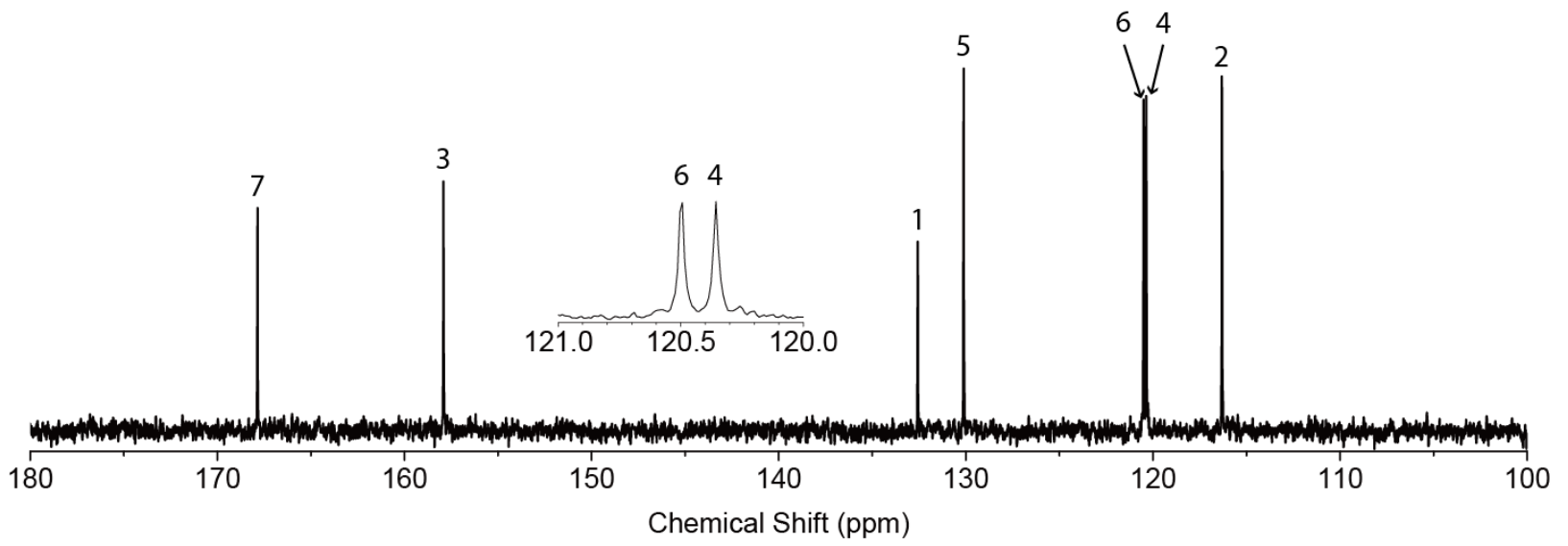

Figure S1b: ${ }^{13} \mathrm{C}$ NMR data obtained from 3-hydroxybenzoic acid. ${ }^{13} \mathrm{C}$ NMR (100 MHz; DMSO$\left.\mathrm{d}_{6}, \mathrm{ppm}\right): \delta 167.9,157.9,132.6,130.1,120.5,120.4,116.3$. 


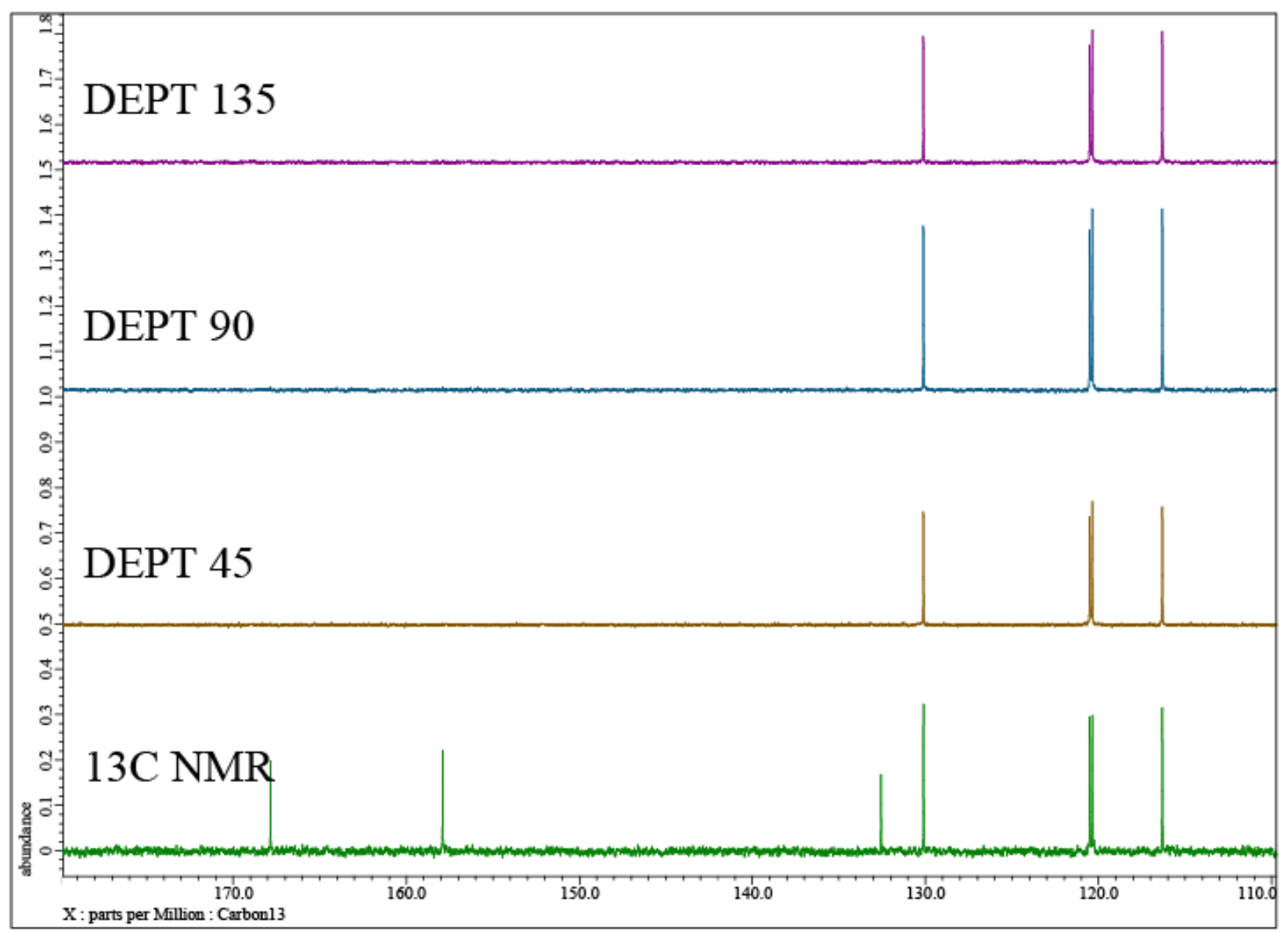

Figure S1c: ${ }^{13} \mathrm{C}$ NMR, DEPT 45, 90, 135 data obtained from 3-hydroxybenzoic acid. 


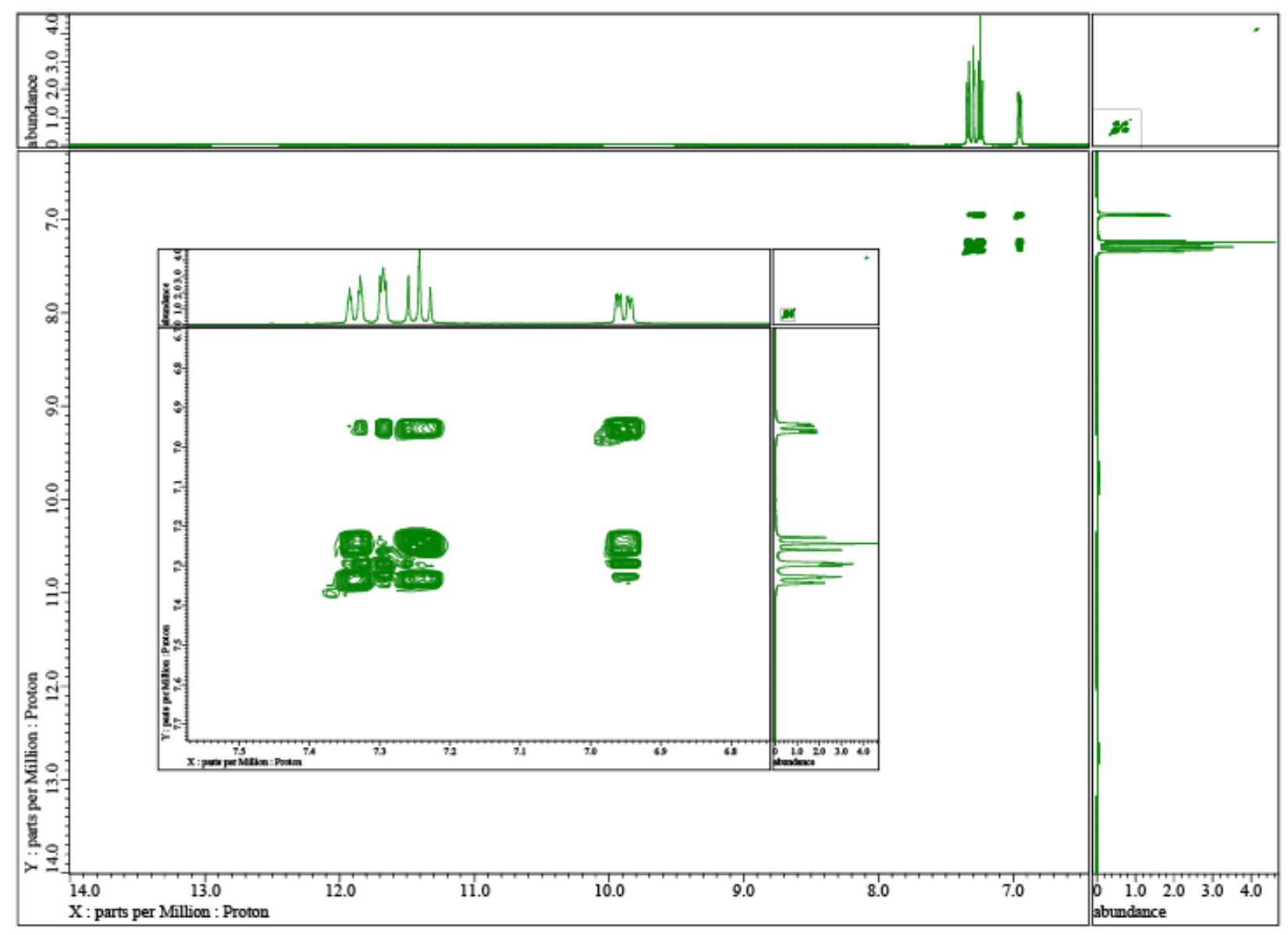

Figure S1d: COSY data obtained from 3-hydroxybenzoic acid. 


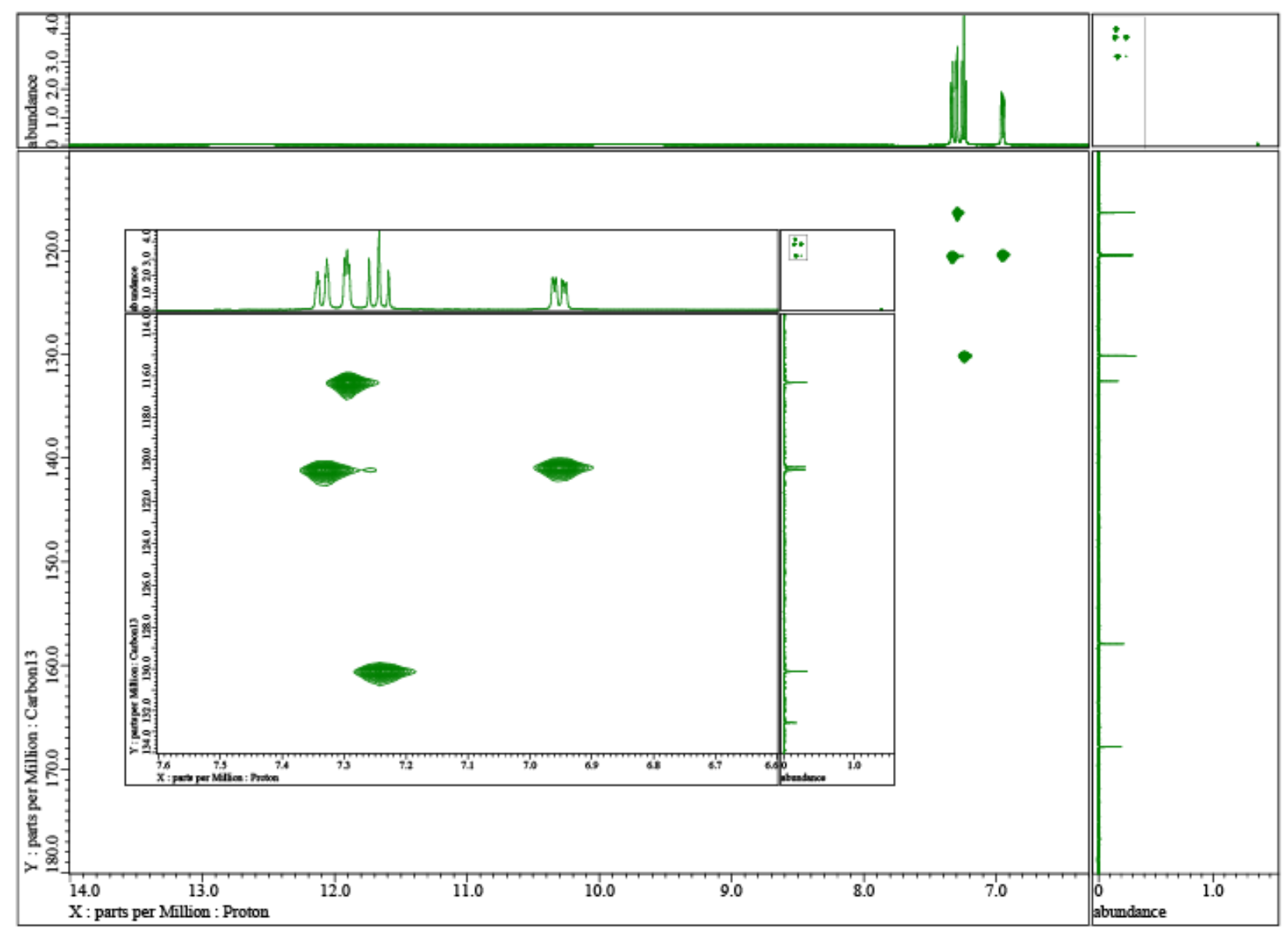

Figure S1e: HSQC data obtained from 3-hydroxybenzoic acid. 


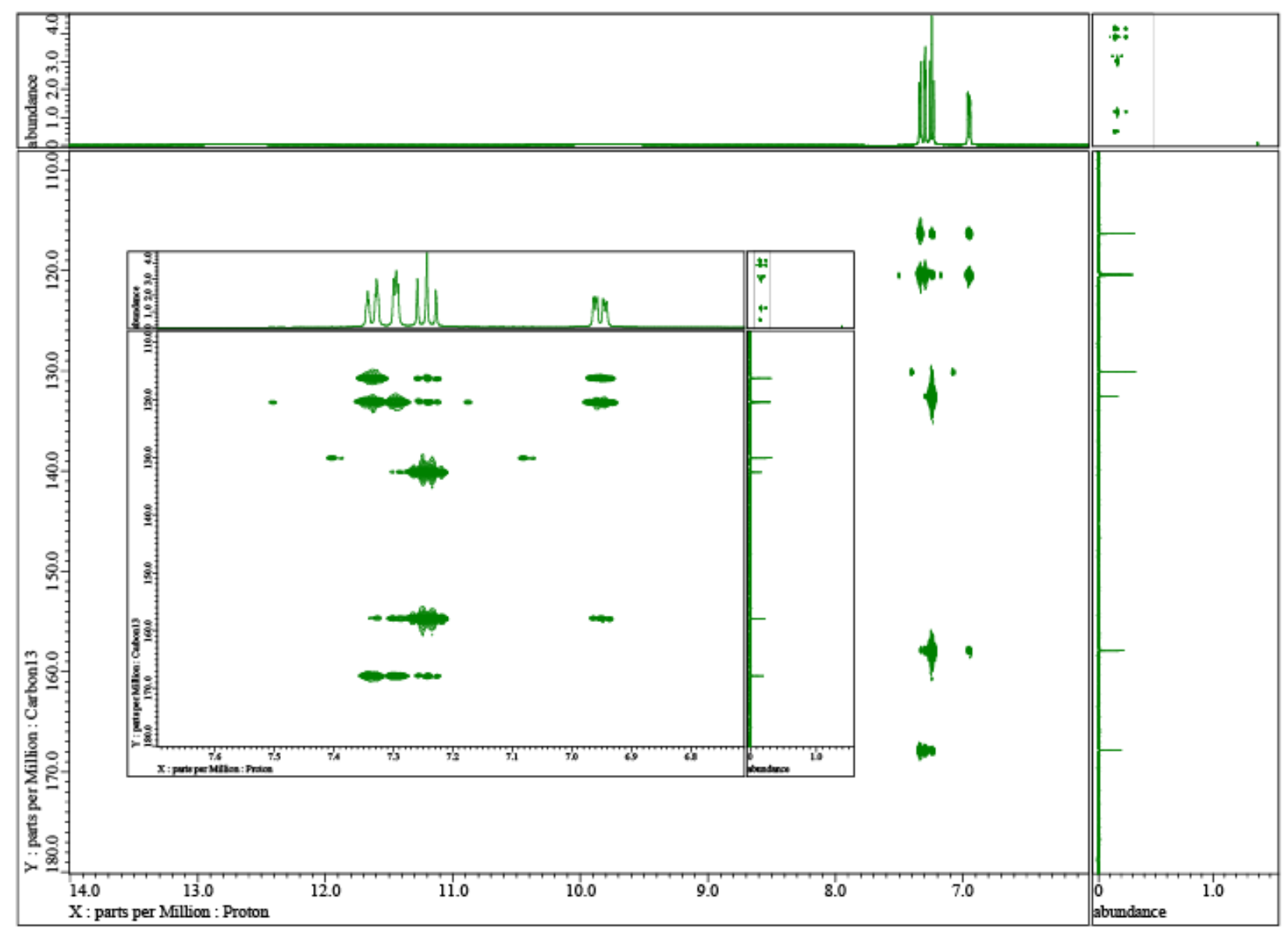

Figure S1f: HMBC data obtained from 3-hydroxybenzoic acid. 


\section{Section 2: NMR Characterization of Allylated 3-Hydroxybenzoic Acid (a3HBA)}

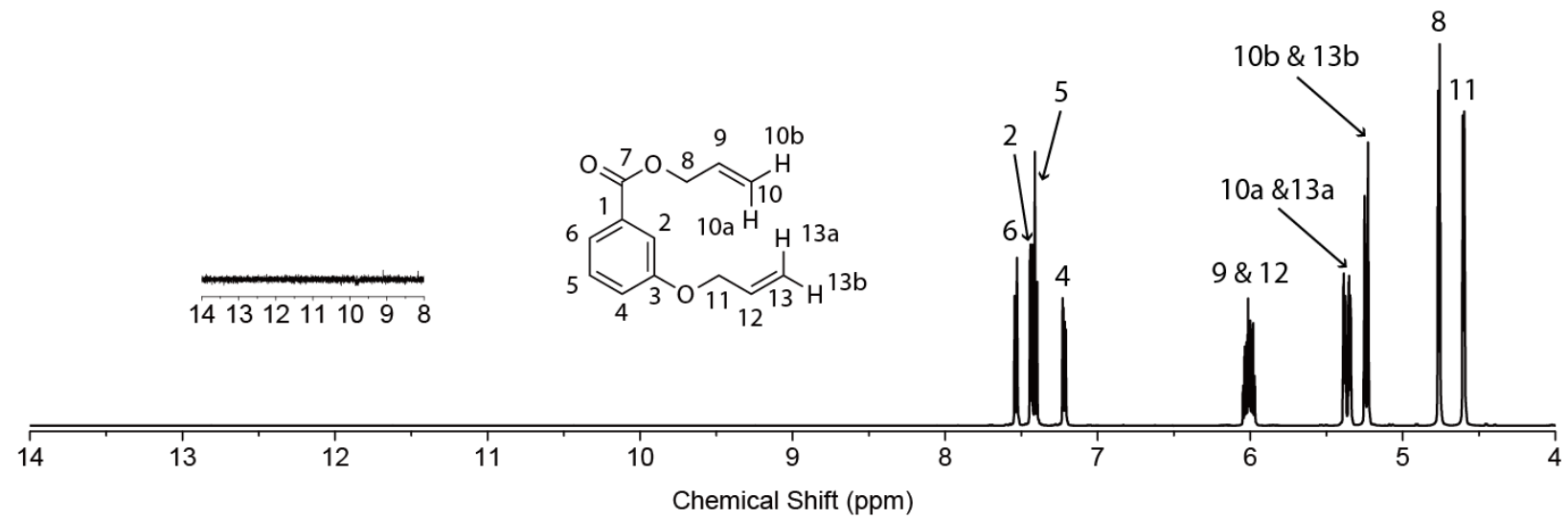

Figure S2a: Chemical structure of and ${ }^{1} \mathrm{H}$ NMR data obtained from allyl 3-allyloxybenzoate (referred to as "allylated 3HBA" in main text). Data were obtained from final purified product. ${ }^{1} \mathrm{H}$ NMR (400 MHz, DMSO-d 6 , ppm): $\delta 7.54$ (ddd, $\left.J=7.79,1.37,1.37 \mathrm{~Hz}, 1 \mathrm{H}\right), 7.44$ (dd, $J=2.75$, $1.83 \mathrm{~Hz}, 1 \mathrm{H}), 7.42$ (dd, $J=7.79,7.79 \mathrm{~Hz}, 1 \mathrm{H}), 7.23$ (ddd, $J=8.24,2.75,0.92 \mathrm{~Hz}, 1 \mathrm{H}), 6.06-5.96$ (m, 2H), 5.40-5.34 (m, 2H), 5.24 (ddt, $J=10.53,1.37,1.37 \mathrm{~Hz}, 2 \mathrm{H}), 4.77$ (ddd, $J=5.50,1.37$, $1.37 \mathrm{~Hz}, 2 \mathrm{H}), 4.61$ (ddd, $J=5.04,1.37,1.37 \mathrm{~Hz}, 2 \mathrm{H})$.
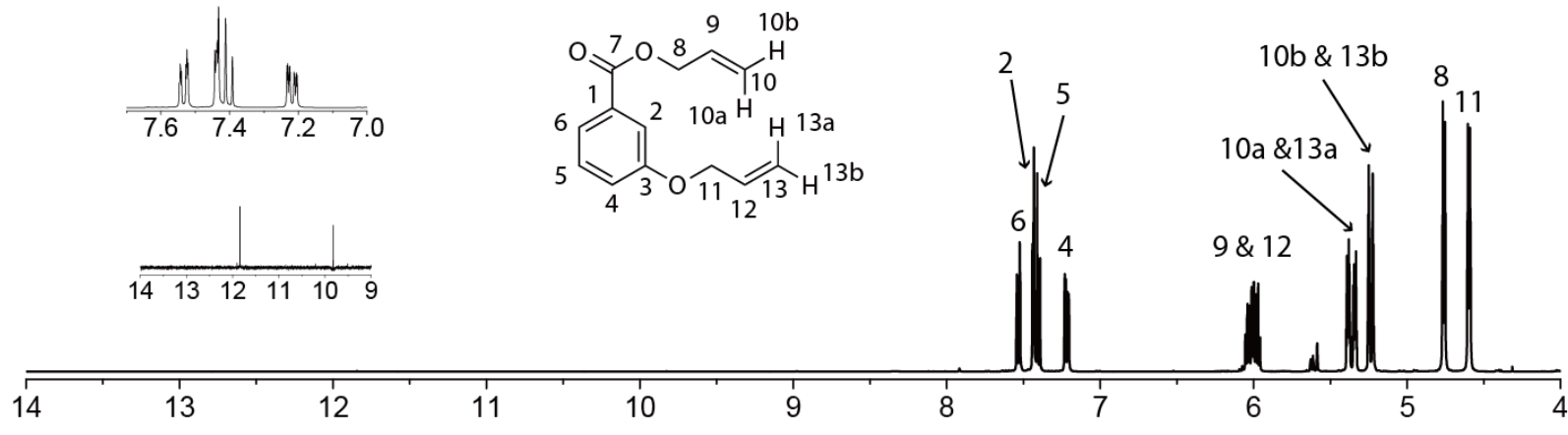

Chemical Shift (ppm)

Figure S2b: Chemical structure of and ${ }^{1} \mathrm{H}$ NMR data obtained from allyl 3-allyloxybenzoate. Data were obtained prior to extraction. Refer to Figure S2c for a closer view. 


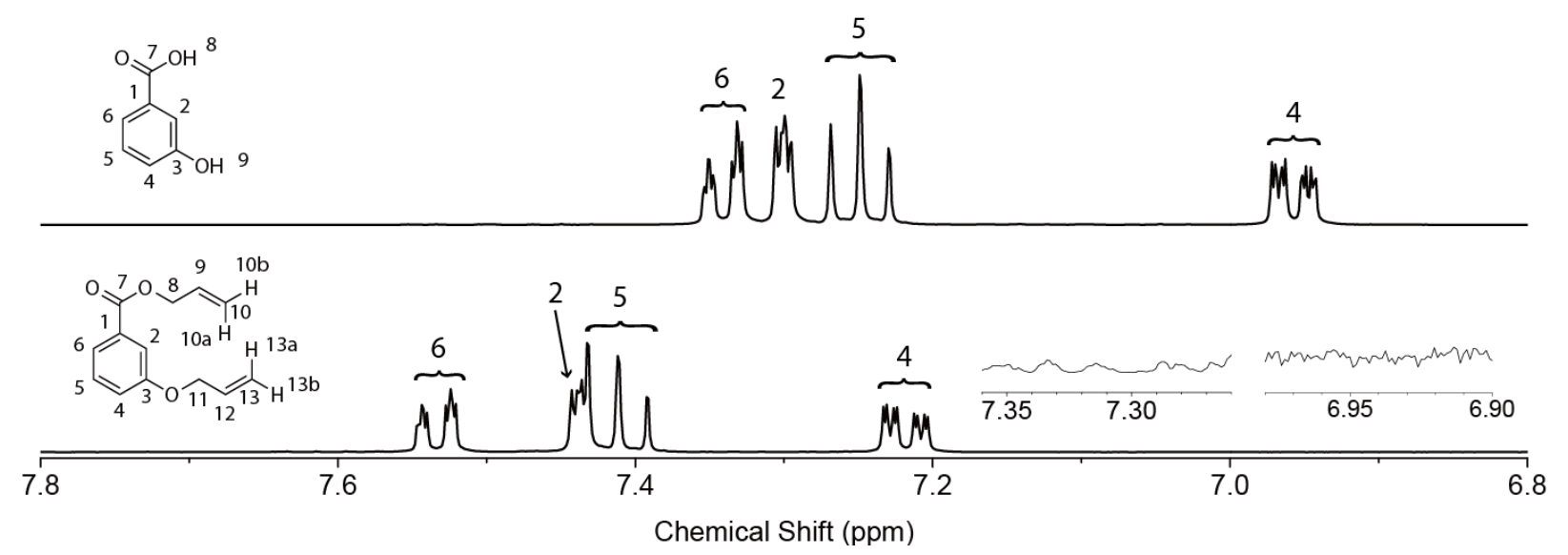

Figure S2c: ${ }^{1} \mathrm{H}$ NMR data obtained from (top) 3-hydroxybenzoic acid and (bottom) allyl 3allyloxybenzoate. The spectrum of allyl 3-allyloxybenzoate was obtained prior to extraction for the purposes of determining the reaction conversion. Using the peak integrals for protons 2,4 and 6 , the reaction conversion was determined to be $97.5 \%$.

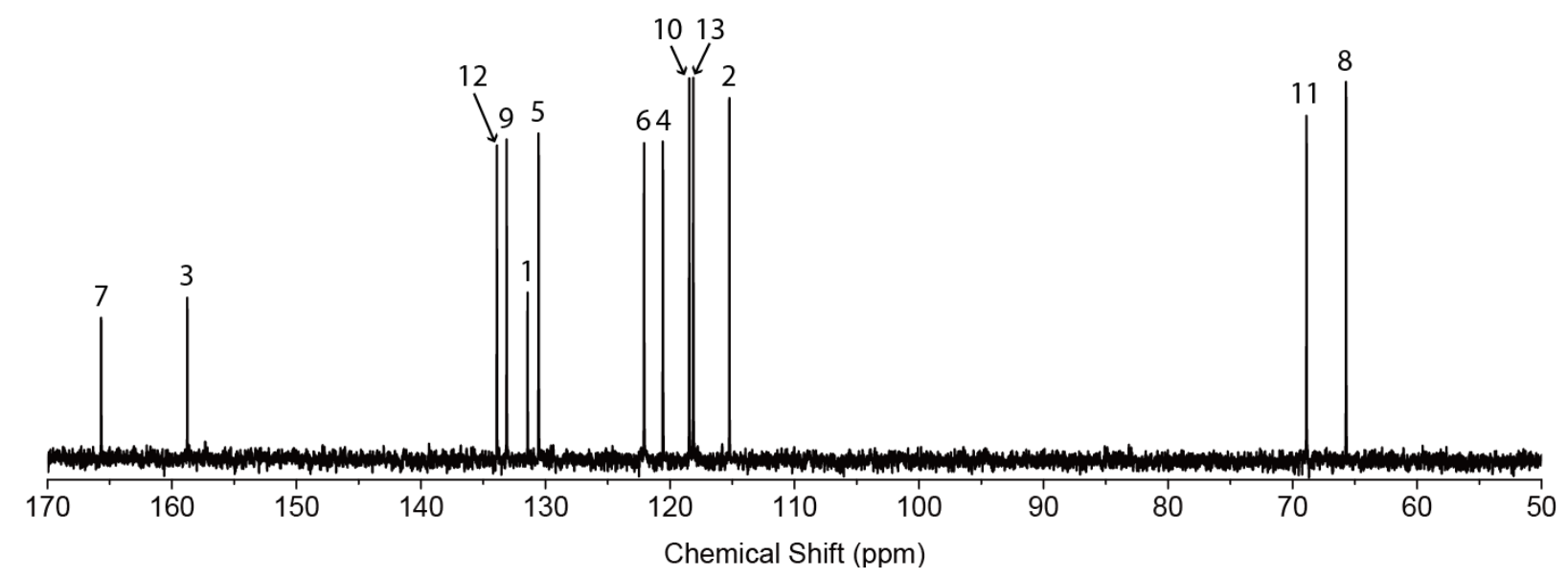

Figure S2d: ${ }^{13} \mathrm{C}$ NMR data obtained from allyl 3-allyloxybenzoate. ${ }^{13} \mathrm{C}$ NMR $(100 \mathrm{MHz}$; DMSO$\left.\mathrm{d}_{6}, \mathrm{ppm}\right): \delta 165.7,158.8,133.9,133.1,131.4,130.6,122.1,120.6,118.5,118.1,115.2,68.9,65.7$. 


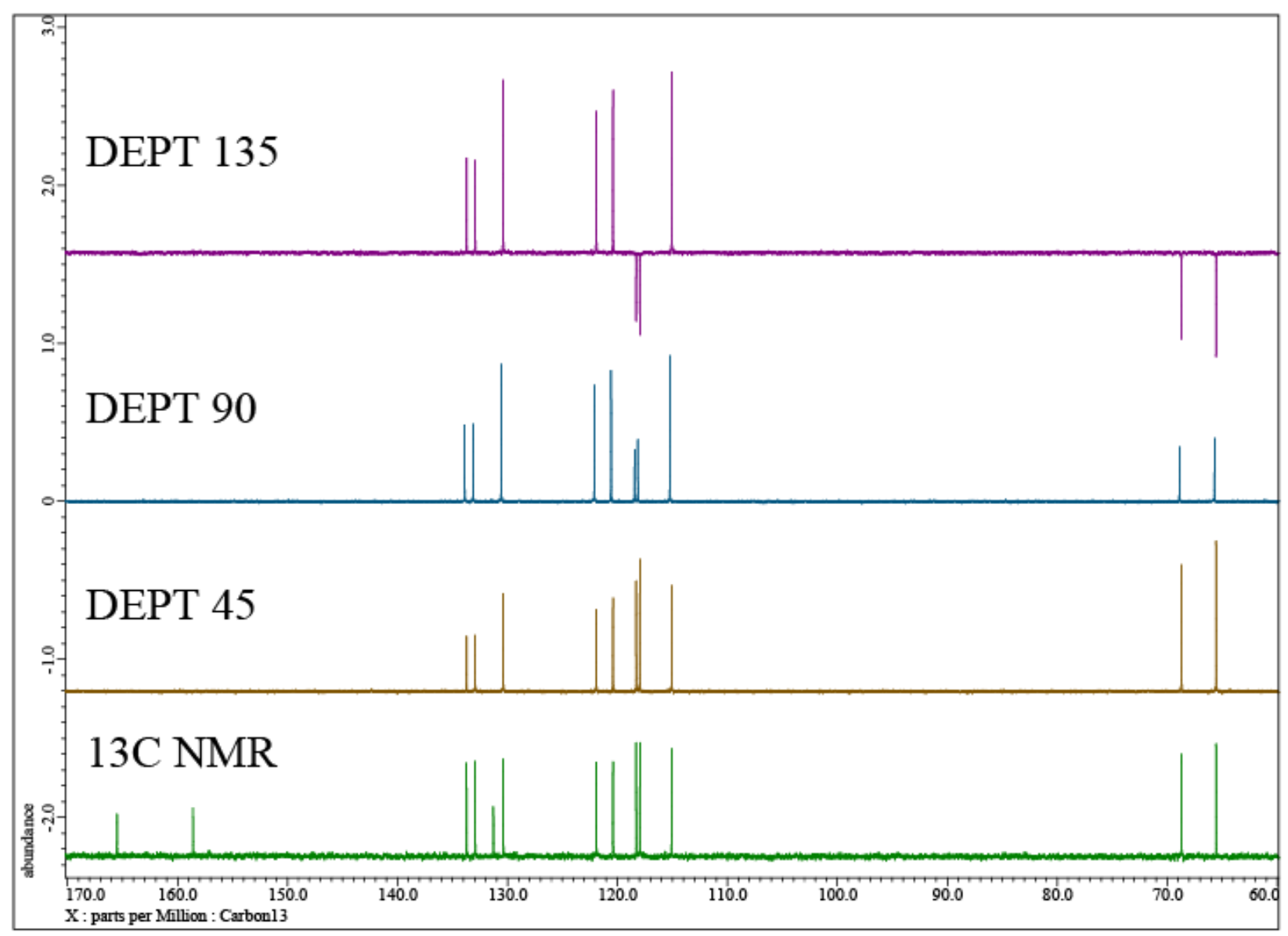

Figure S2e: ${ }^{13} \mathrm{C}$ NMR, DEPT 45, 90, 135 data obtained from allyl 3-allyloxybenzoate. 


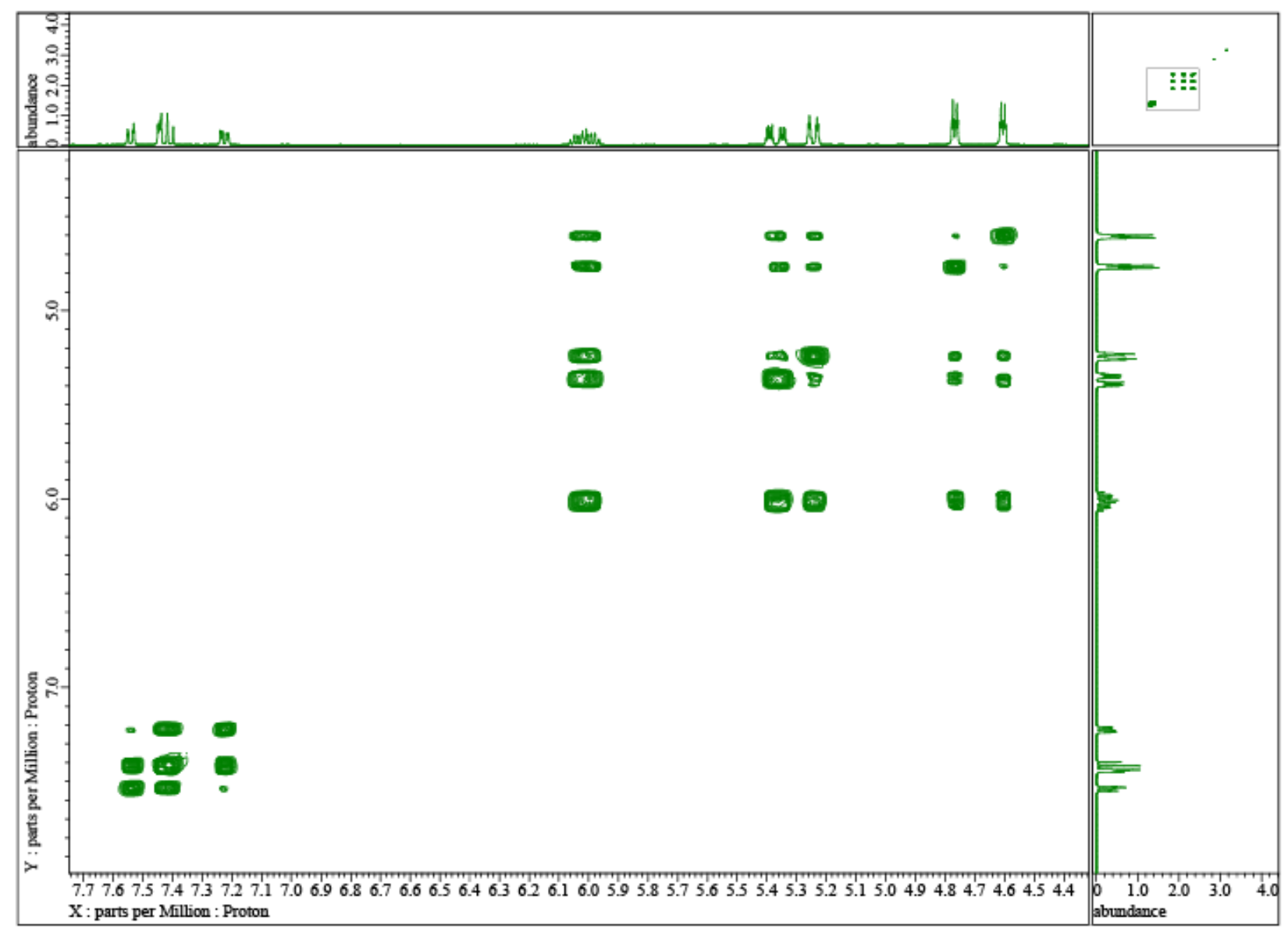

Figure S2f: COSY data obtained from allyl 3-allyloxybenzoate. 


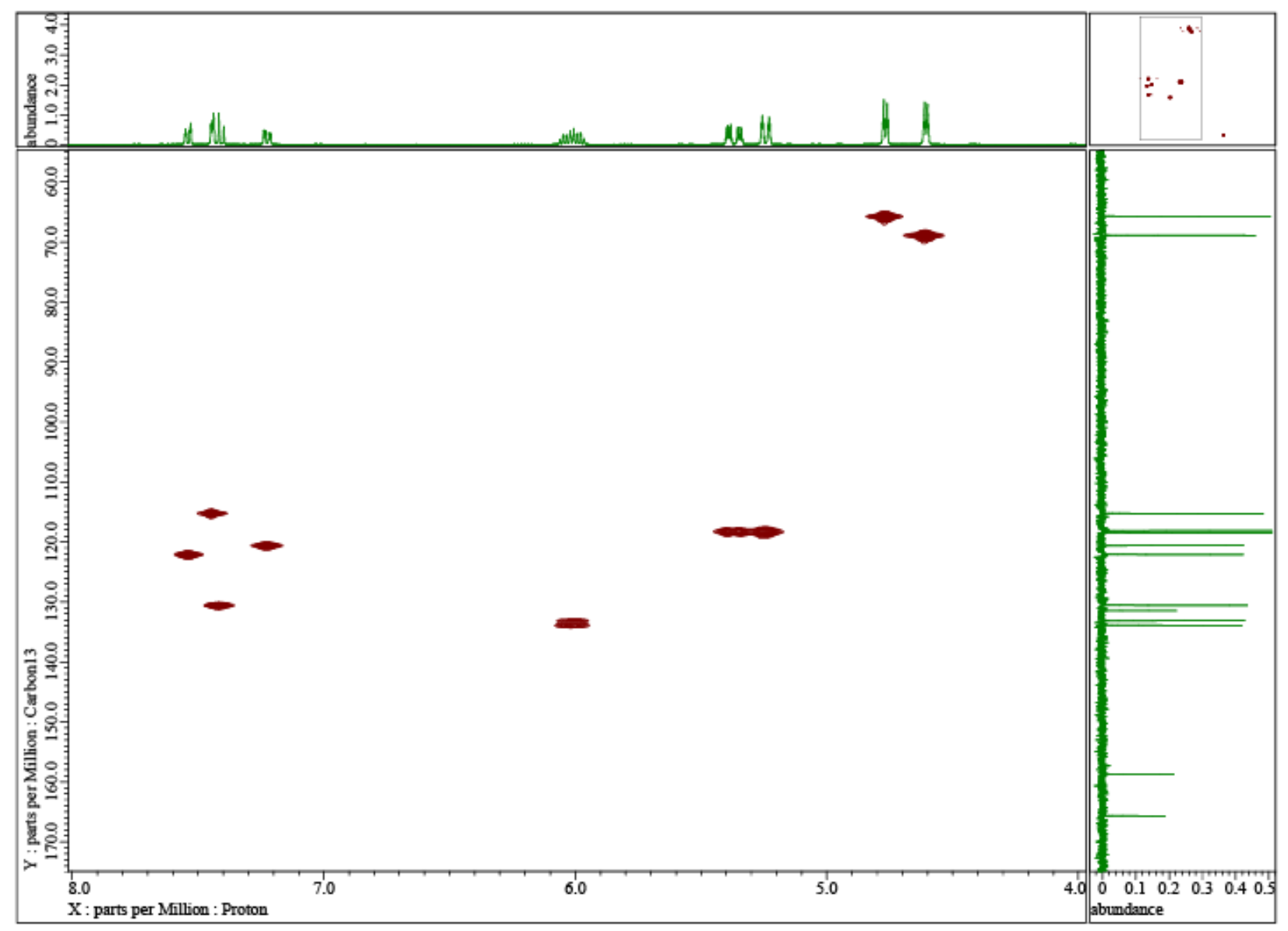

Figure S2g: HSQC data obtained from allyl 3-allyloxybenzoate. 


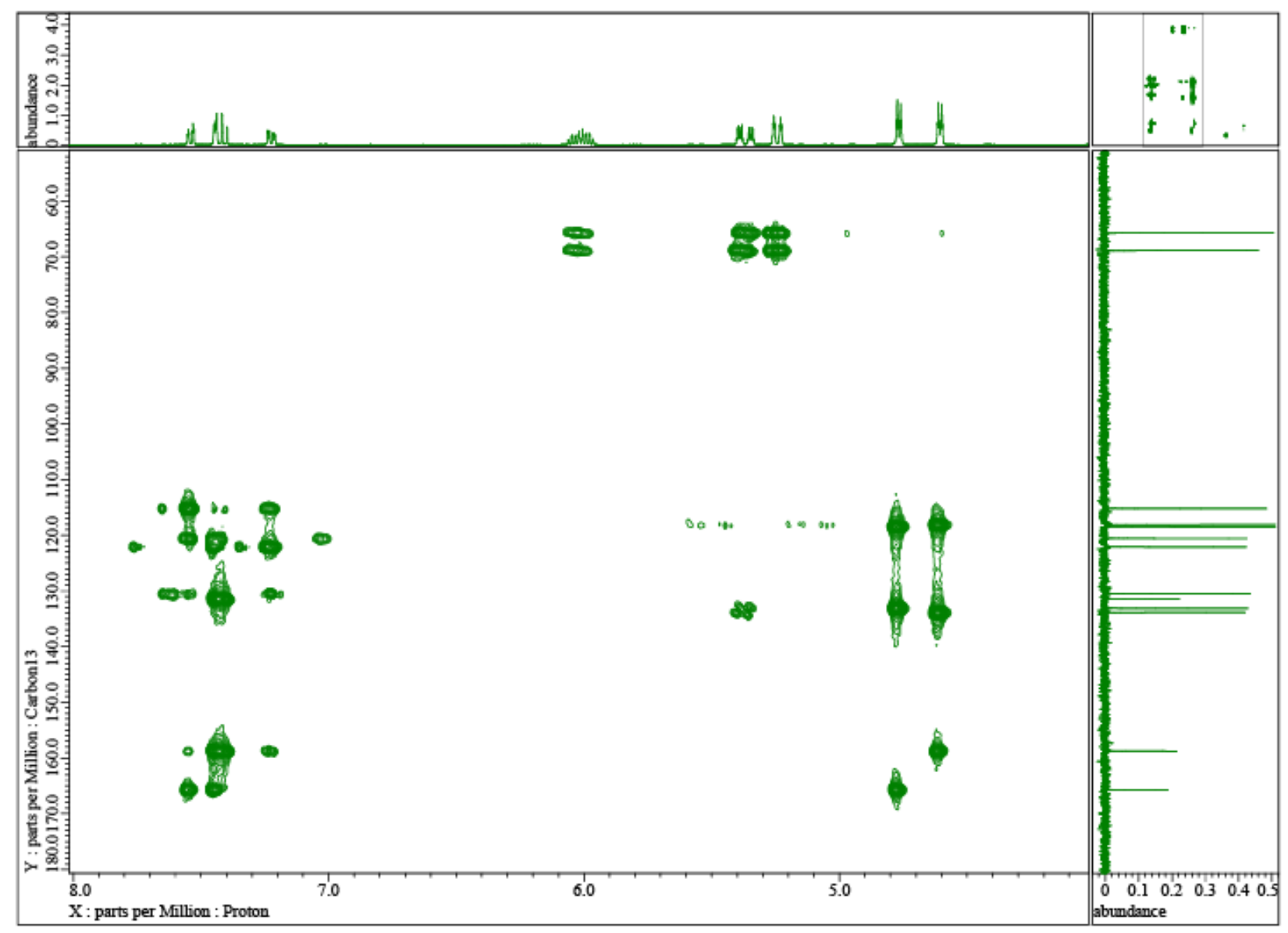

Figure S2h: HMBC data obtained from allyl 3-allyloxybenzoate. 


\section{Section 3: NMR Characterization of Gentisic Acid}
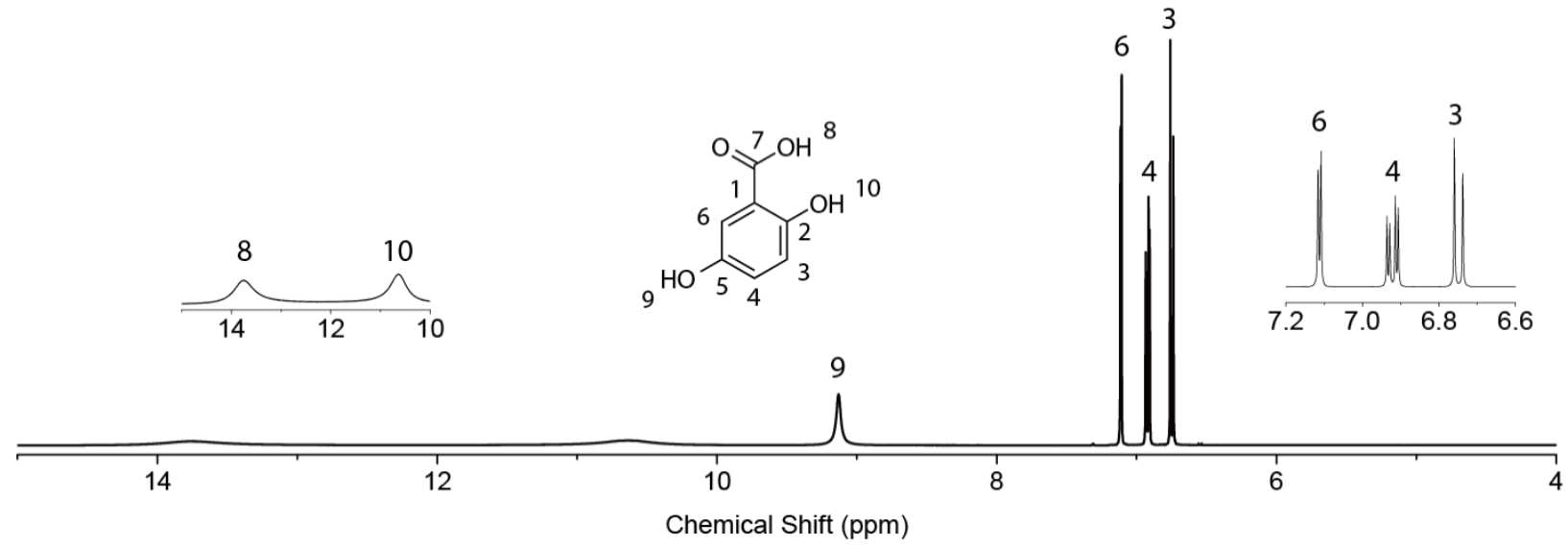

Figure S3a: Chemical structure of and ${ }^{1} \mathrm{H}$ NMR data obtained from gentisic acid (referred to as "GenA" in main text). ${ }^{1} \mathrm{H}$ NMR (400 MHz, DMSO-d 6 , ppm): $\delta 13.75$ (broad s, 1H), 10.65 (broad s, 1H), $9.13(\mathrm{~s}, 1 \mathrm{H}), 7.11(\mathrm{~d}, J=2.93 \mathrm{~Hz}, 1 \mathrm{H}), 6.92(\mathrm{dd}, J=8.79,2.93 \mathrm{~Hz}, 1 \mathrm{H}), 6.75(\mathrm{~d}, J=8.79$ $\mathrm{Hz}, 1 \mathrm{H})$.

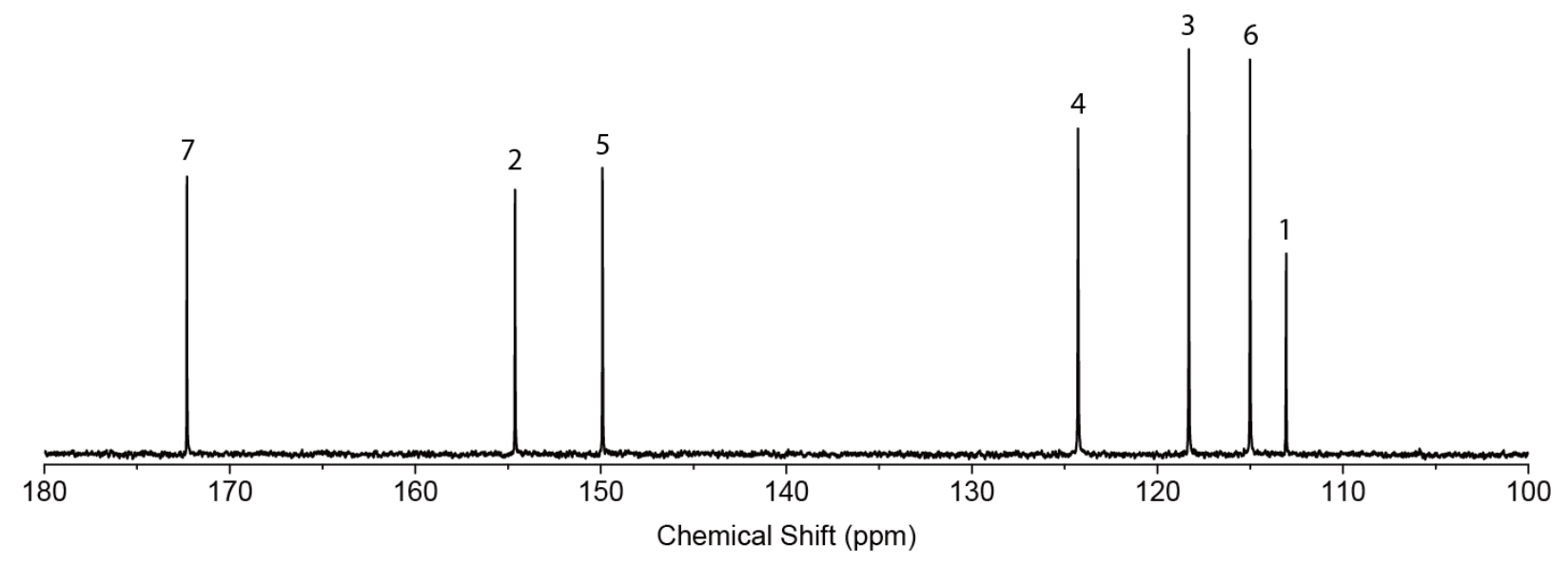

Figure S3b: ${ }^{13} \mathrm{C}$ NMR data obtained from gentisic acid. ${ }^{13} \mathrm{C}$ NMR (100 MHz; DMSO-d $\left.6, p p m\right): \delta$ $172.3,154.6,149.9,124.3,118.3,115.0,113.1$. 


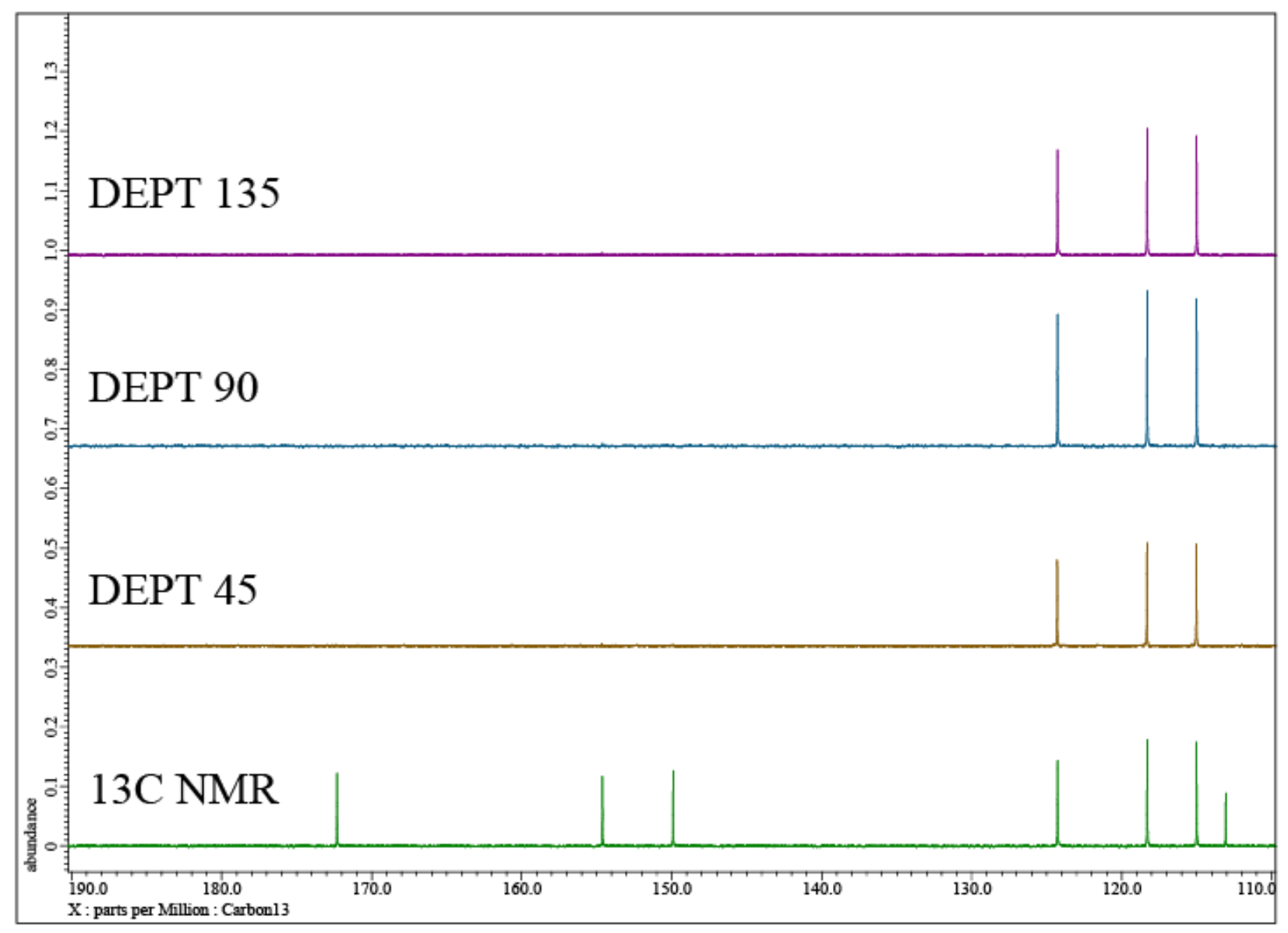

Figure S3c: ${ }^{13} \mathrm{C}$ NMR, DEPT 45, 90, 135 data obtained from gentisic acid. 


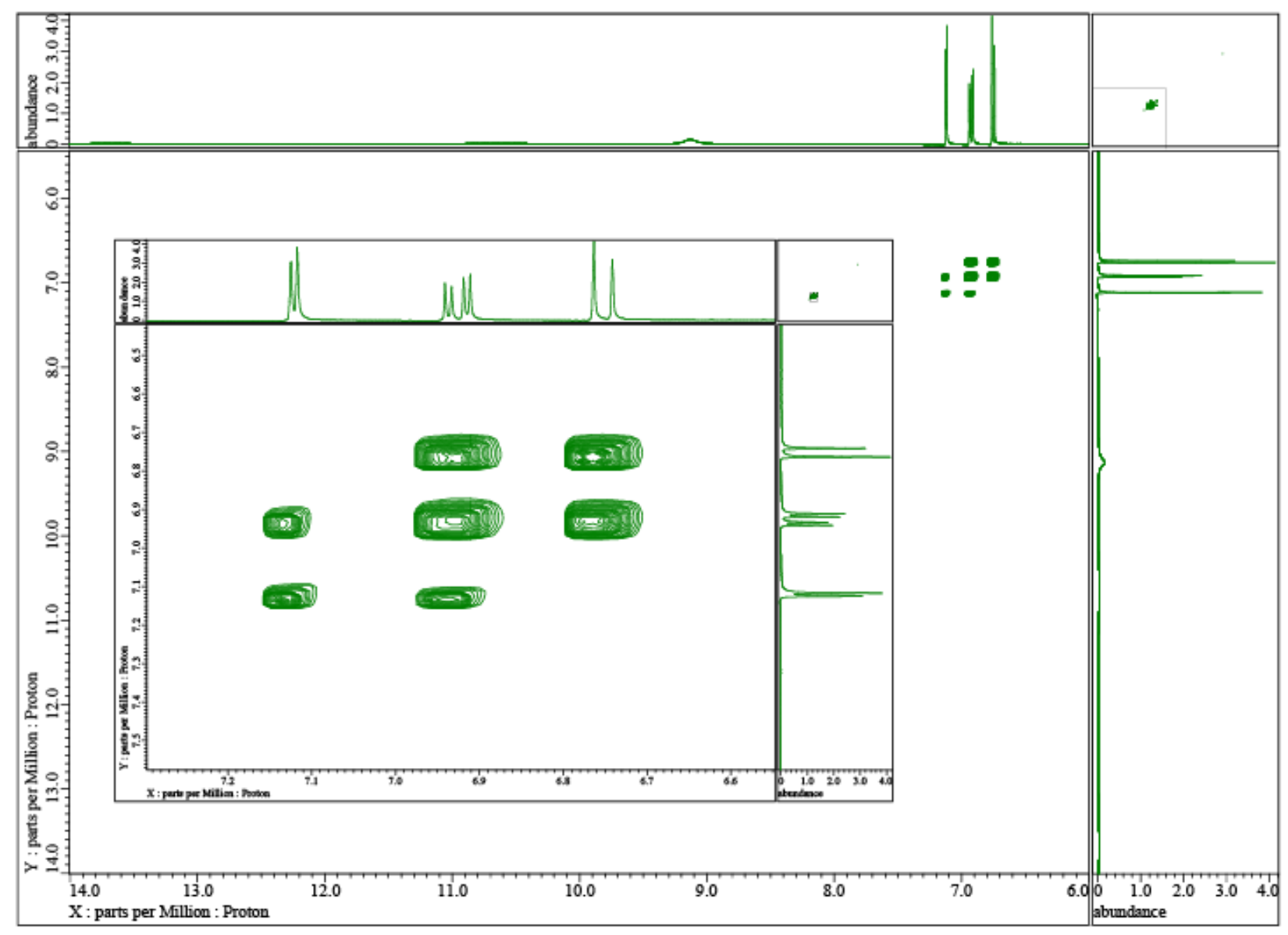

Figure S3d: COSY data obtained from gentisic acid. 


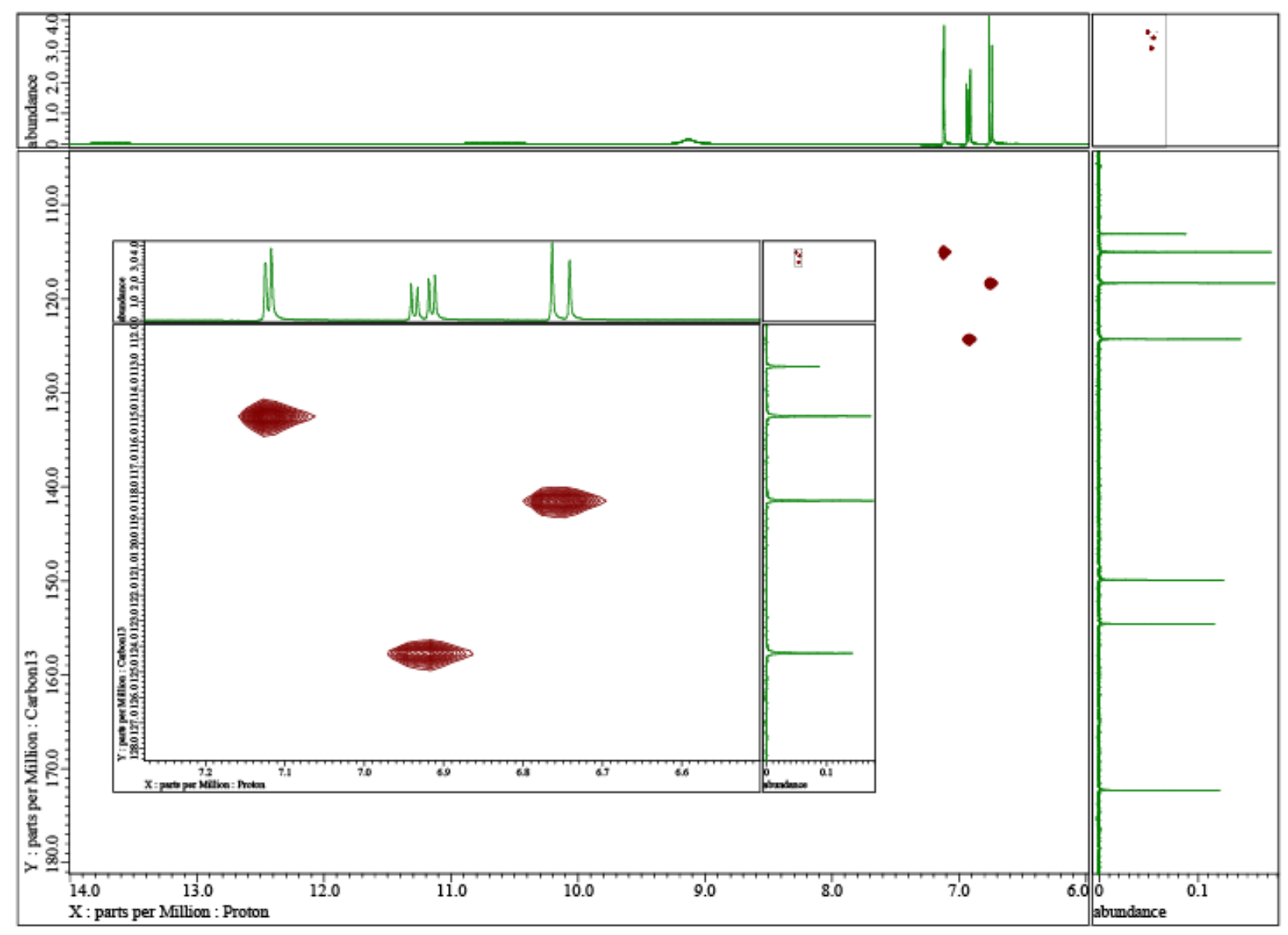

Figure S3e: HSQC data obtained from gentisic acid. 


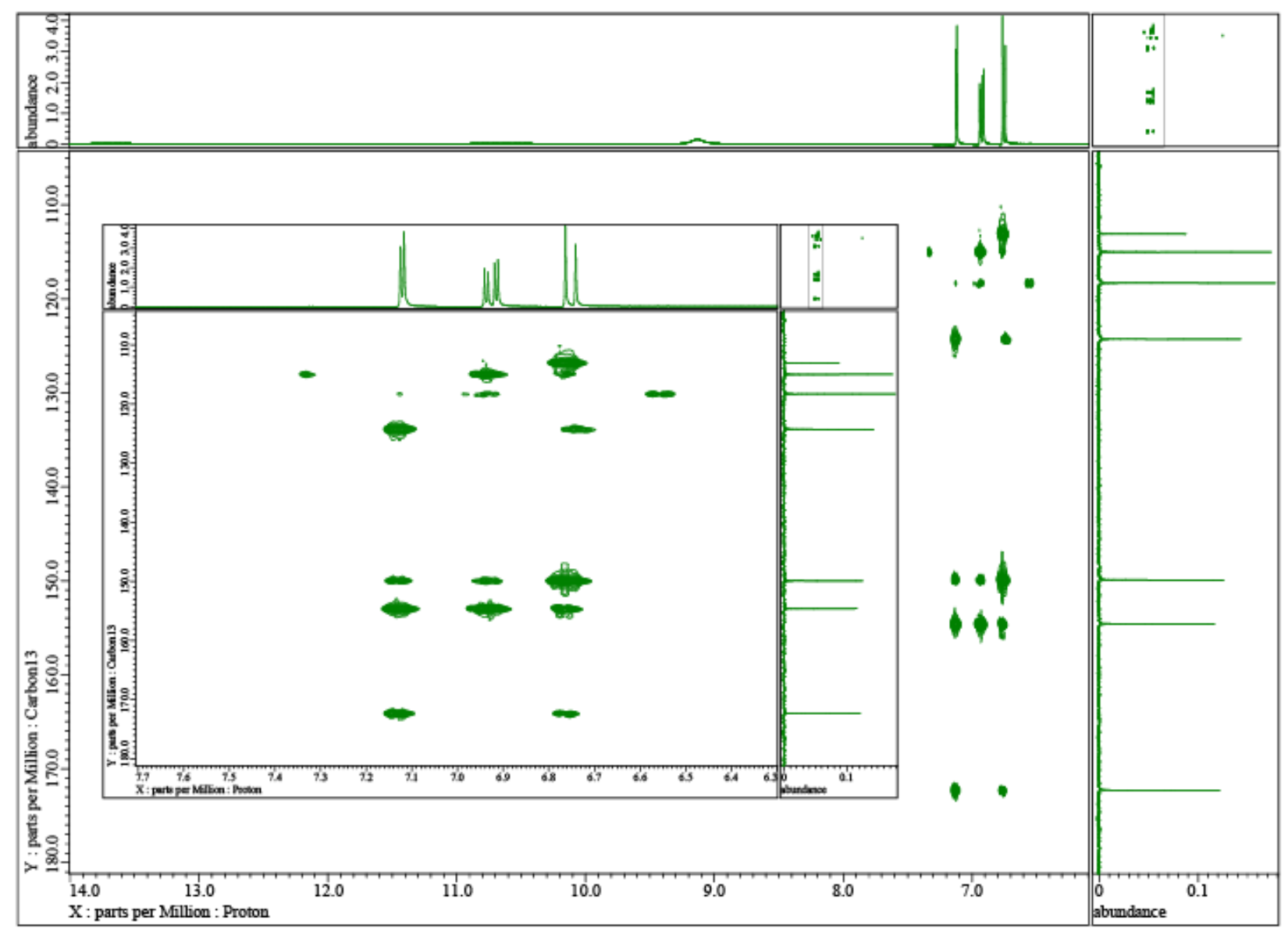

Figure S3f: HMBC data obtained from gentisic acid. 


\section{Section 4: NMR Characterization of Allylated Gentisic Acid (aGenA)}

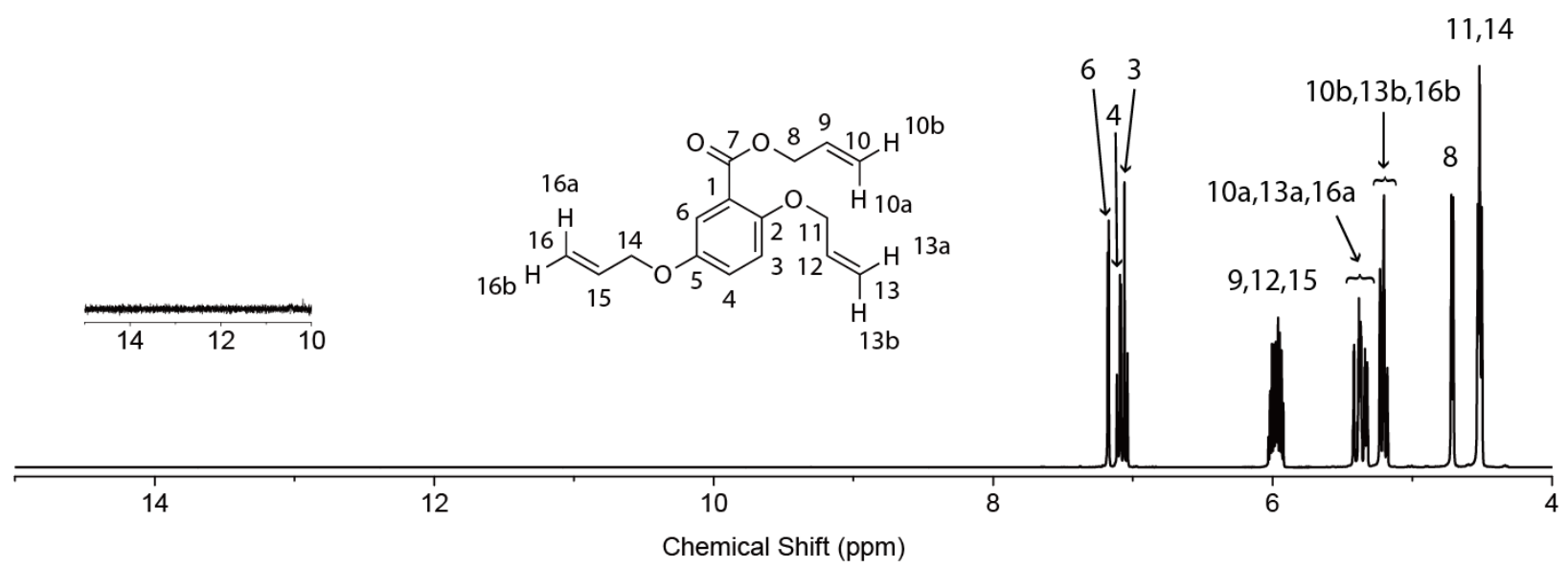

Figure S4a: Chemical structure of and ${ }^{1} \mathrm{H}$ NMR data obtained from allyl 2,5-bis(allyloxy)benzoate (referred to as "allylated GenA" in main text). Data were obtained from final purified product. ${ }^{1} \mathrm{H}$ NMR (400 MHz, DMSO-d, ppm): $\delta 7.18(\mathrm{~d}, J=2.93 \mathrm{~Hz}, 1 \mathrm{H}), 7.10(\mathrm{dd}, J=8.792 .93 \mathrm{~Hz}, 1 \mathrm{H})$, 7.05 (d, $J=8.79 \mathrm{~Hz}, 1 \mathrm{H}), 6.03-5.92$ (m, 3H), 5.43-5.32 (m, 3H), 5.23-5.17 (m, 3H), 4.71 (ddd, $J$ $=5.37,1.47,1.47 \mathrm{~Hz}, 2 \mathrm{H}), 4.54-4.50(\mathrm{~m}, 4 \mathrm{H})$.
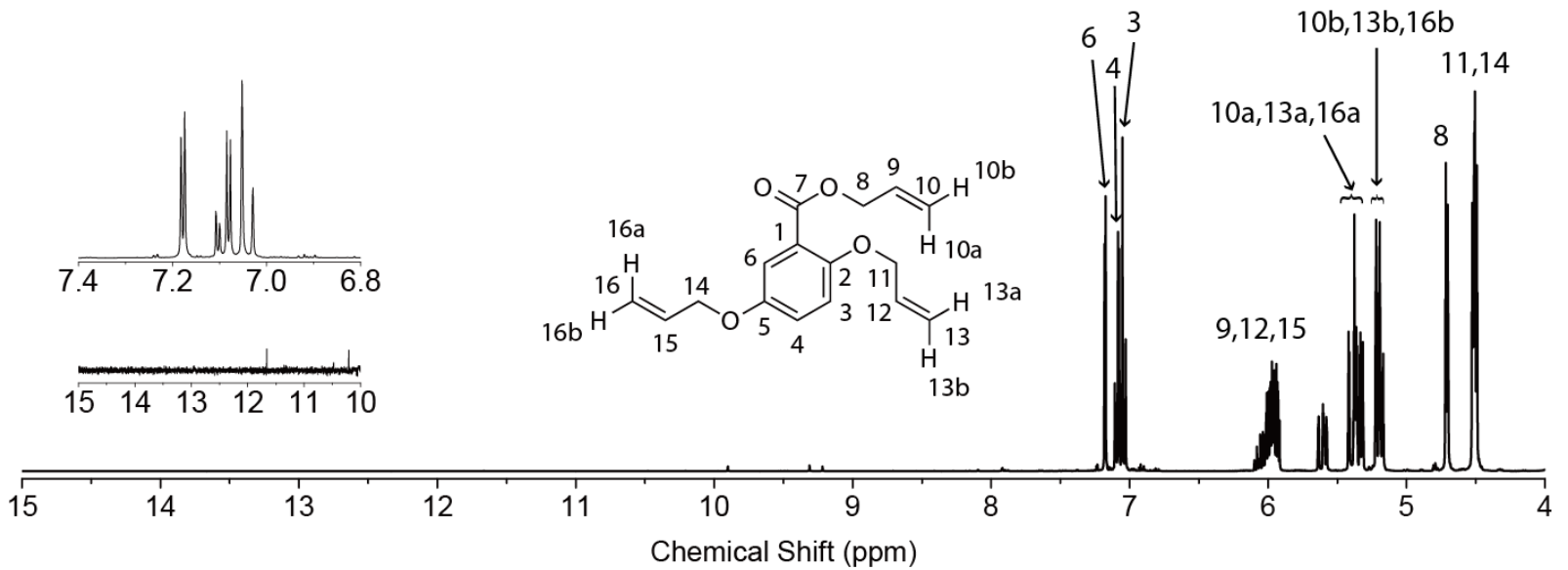

Figure S4b: Chemical structure of and ${ }^{1} \mathrm{H}$ NMR data obtained from allyl 2,5-bis(allyloxy)benzoate. Data were obtained prior to extraction. Refer to Figure $\mathrm{S} 4 \mathrm{c}$ for a closer view. 


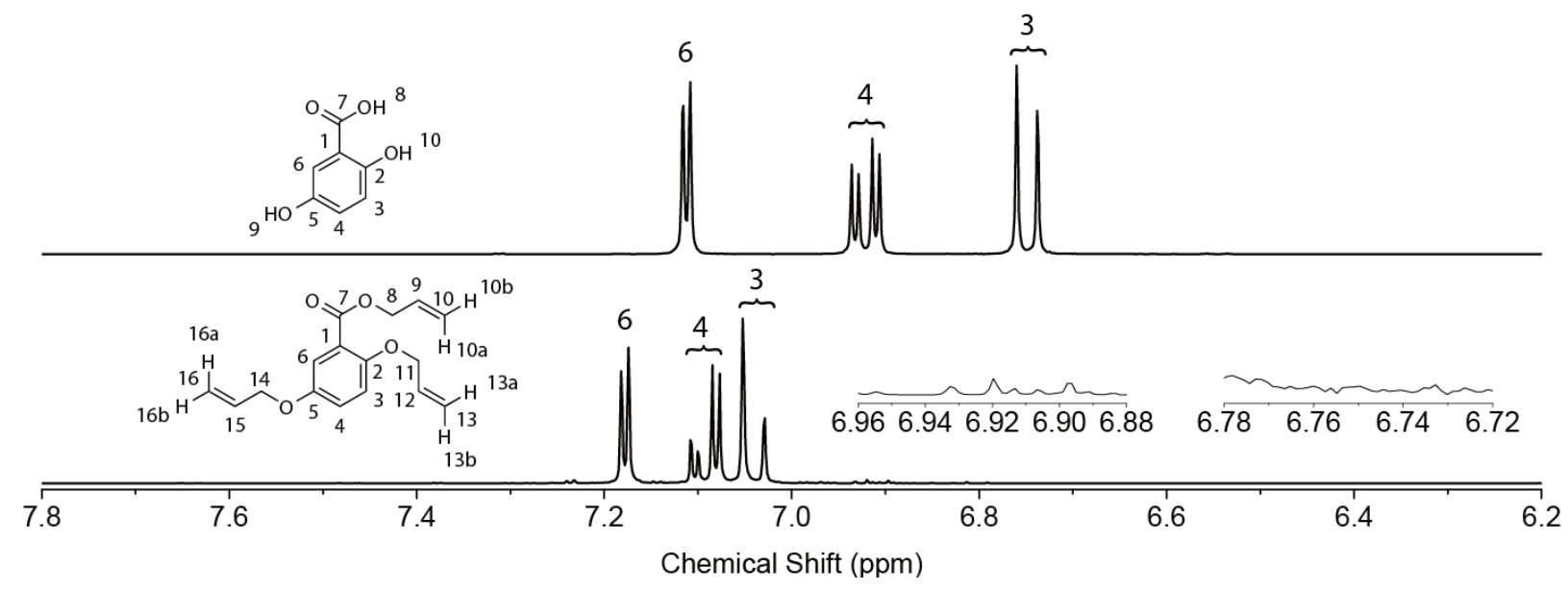

Figure S4c: ${ }^{1} \mathrm{H}$ NMR data obtained from (top) gentisic acid and (bottom) allyl 2,5bis(allyloxy)benzoate. The spectrum of allyl 2,5-bis(allyloxy)benzoate was obtained prior to extraction for the purposes of determining the reaction conversion. Using the peak integrals for protons 3 and 4, the reaction conversion was determined to be $97.5 \%$.

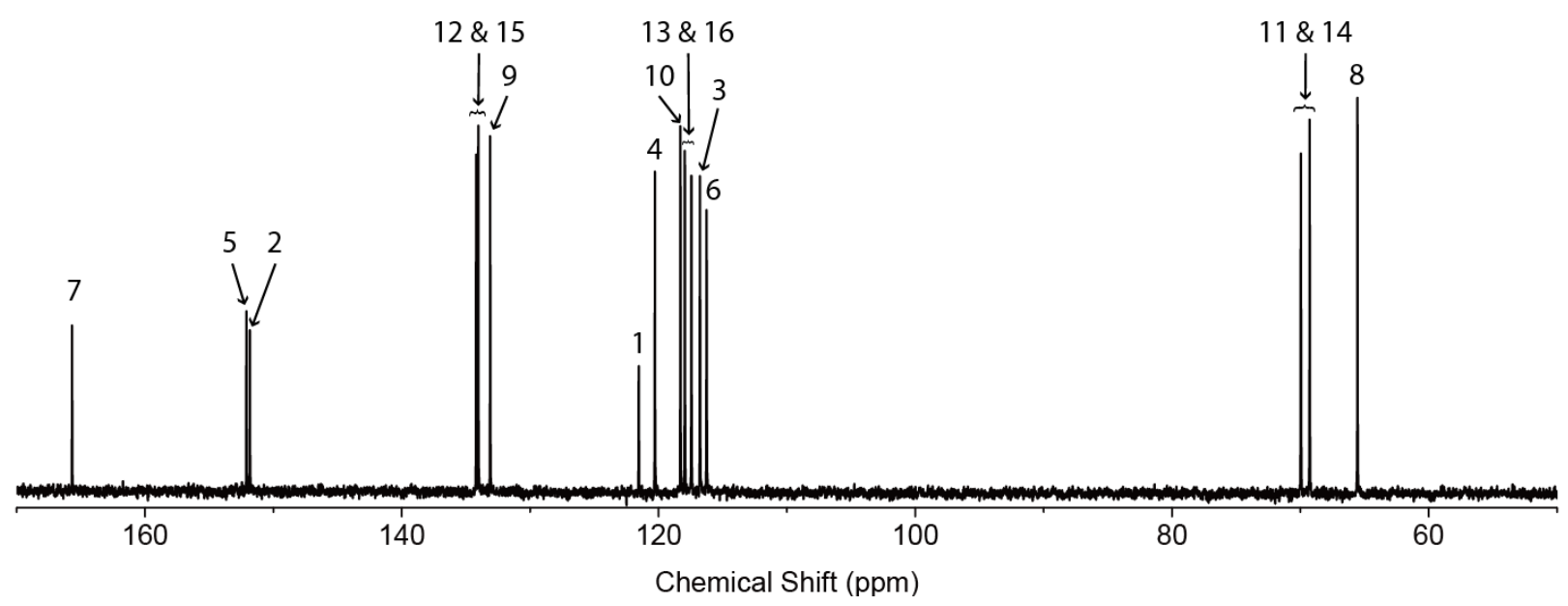

Figure S4d: ${ }^{13} \mathrm{C}$ NMR data obtained from allyl 2,5-bis(allyloxy)benzoate. ${ }^{13} \mathrm{C}$ NMR (100 MHz; DMSO-d $6, \mathrm{ppm}): \delta 165.7,152.1,151.8,134.2,134.0,133.1,121.5,120.3,118.3,118.0,117.4$, $116.8,116.3,70.0,69.3,65.5$. 


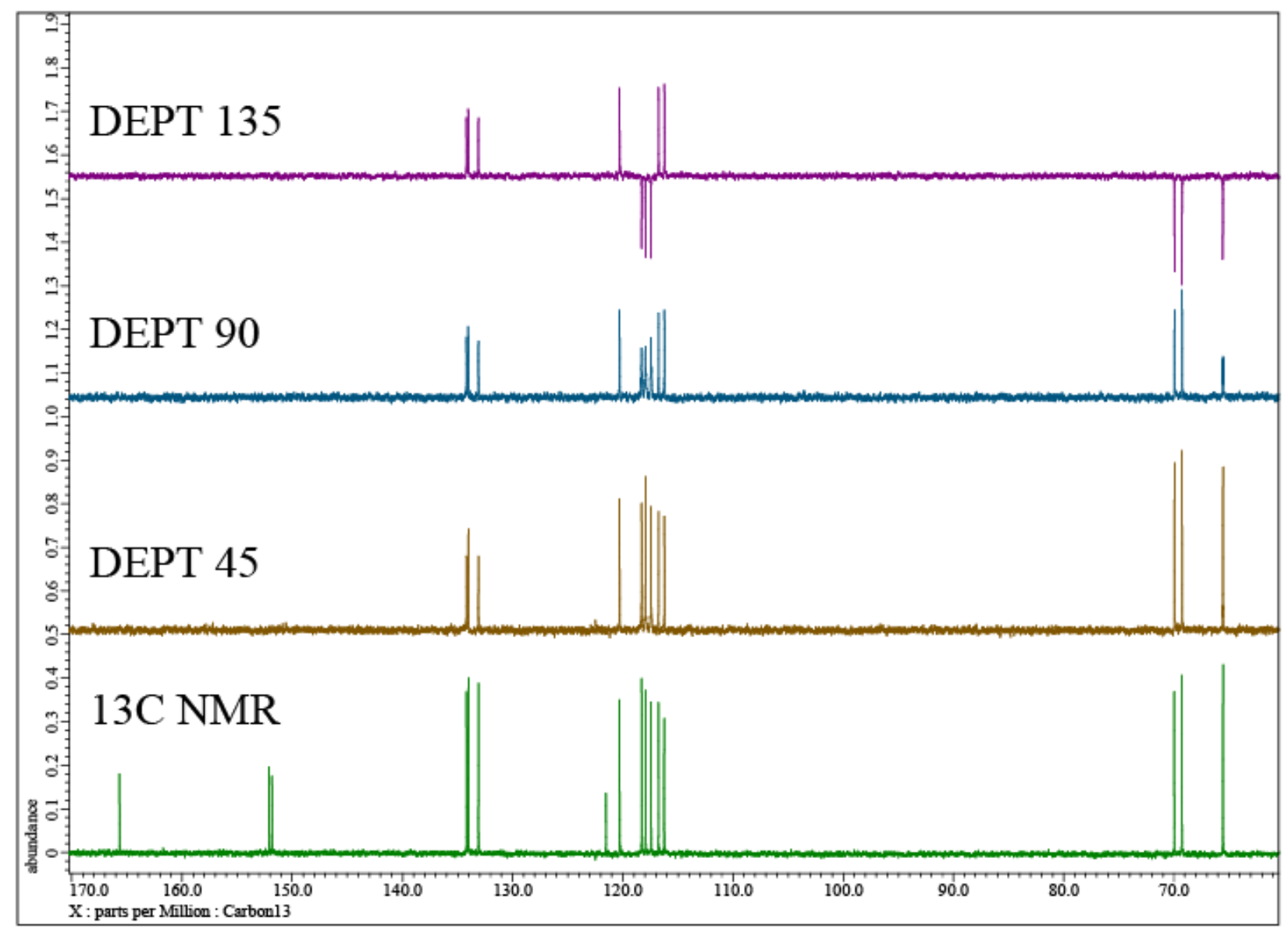

Figure S4e: ${ }^{13} \mathrm{C}$ NMR, DEPT 45, DEPT 90, DEPT 135 data obtained from allyl 2,5bis(allyloxy)benzoate. 


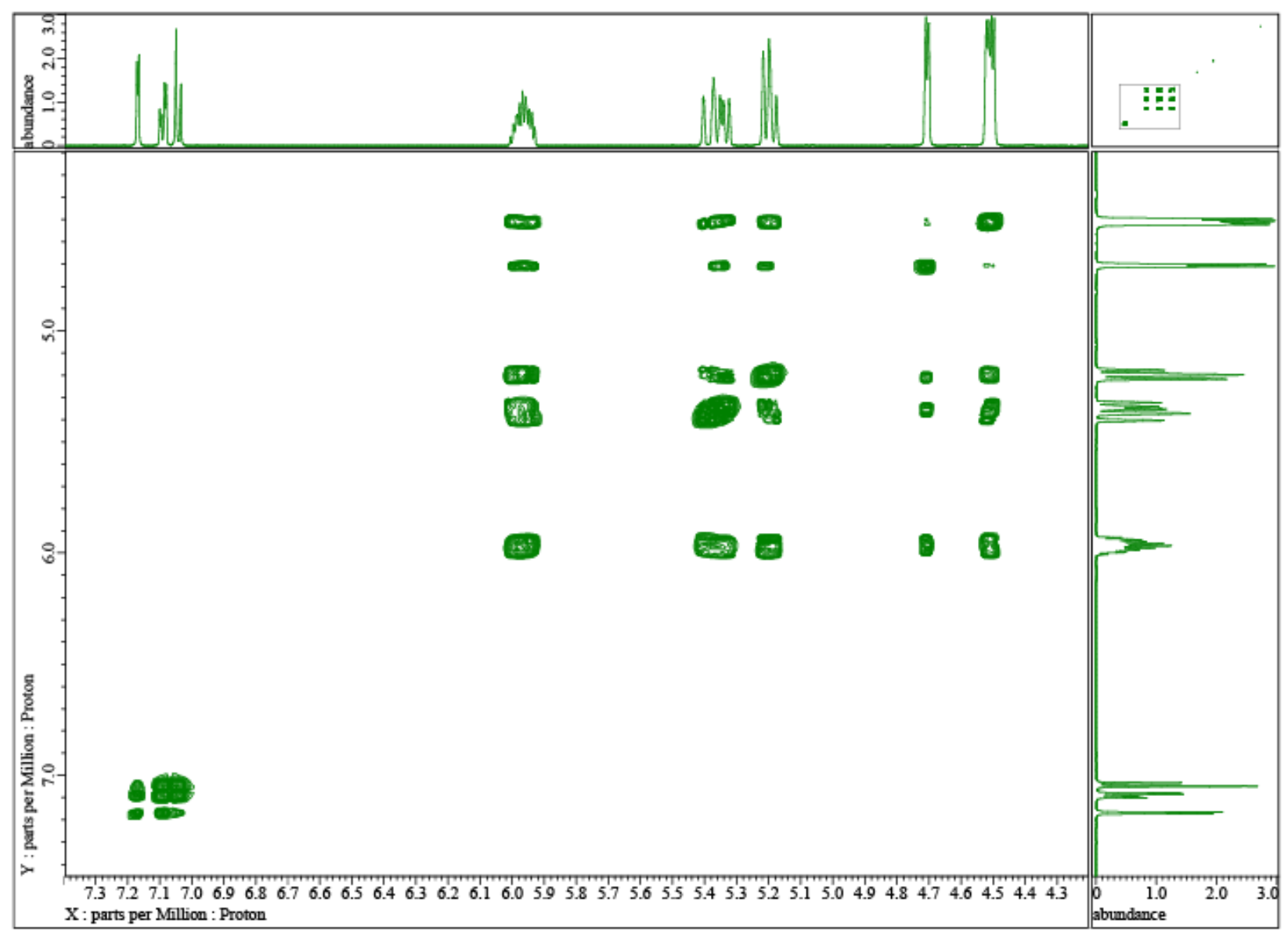

Figure S4f: COSY data obtained from allyl 2,5-bis(allyloxy)benzoate. 


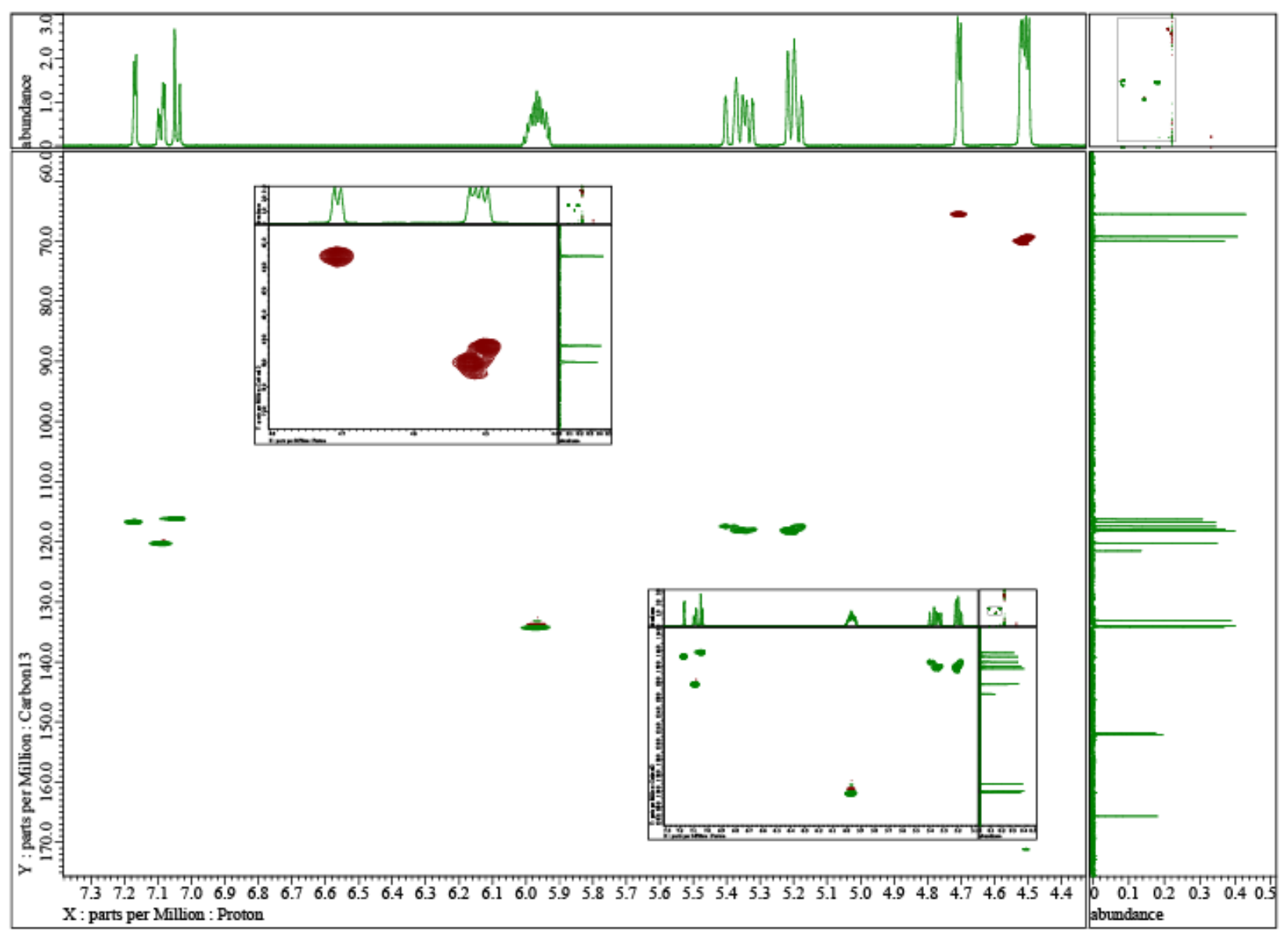

Figure S4g: HSQC data obtained from allyl 2,5-bis(allyloxy)benzoate. 


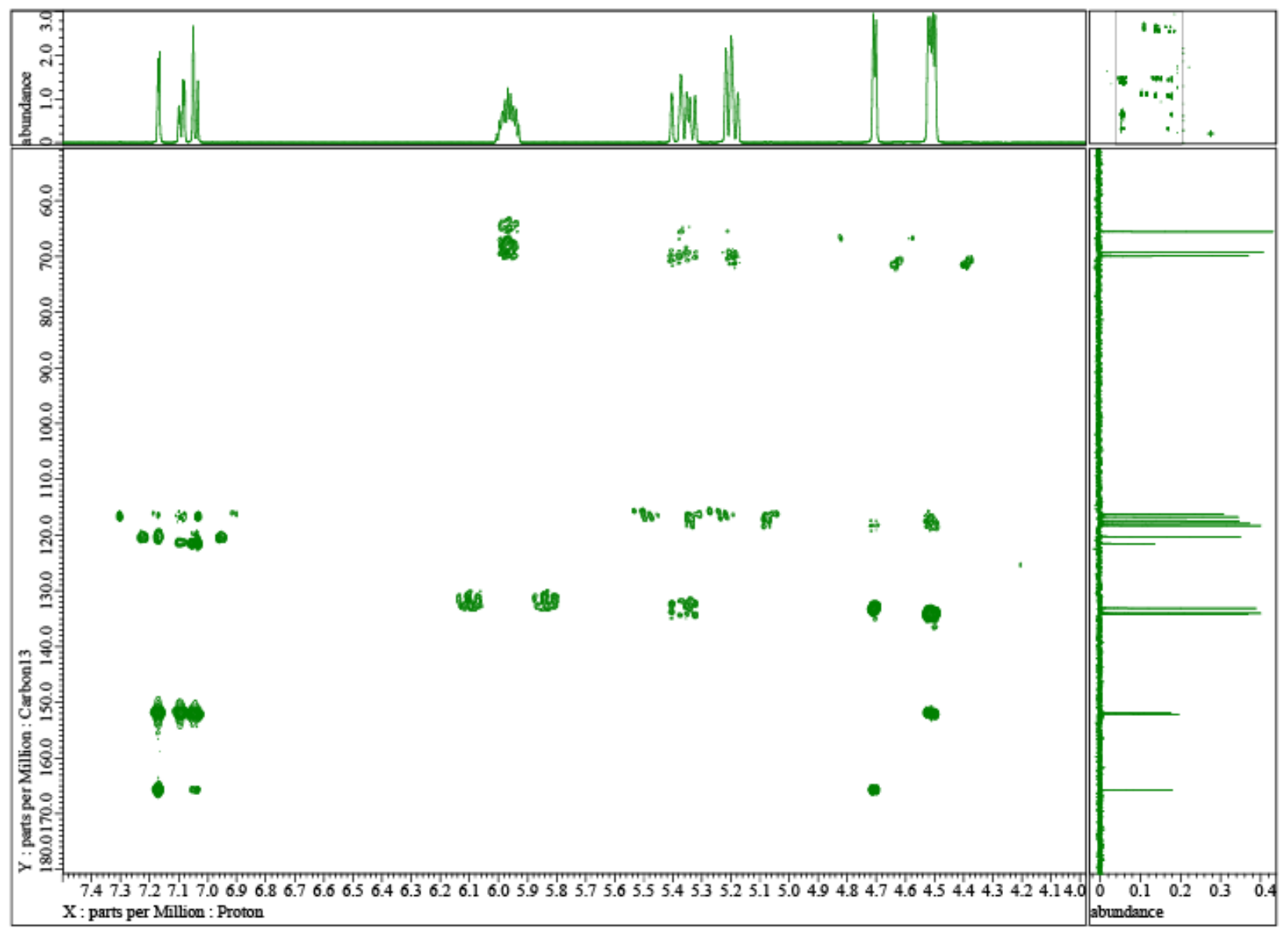

Figure S4h: HMBC data obtained from allyl 2,5-bis(allyloxy)benzoate. 


\section{Section 5: NMR Characterization of Gallic Acid}
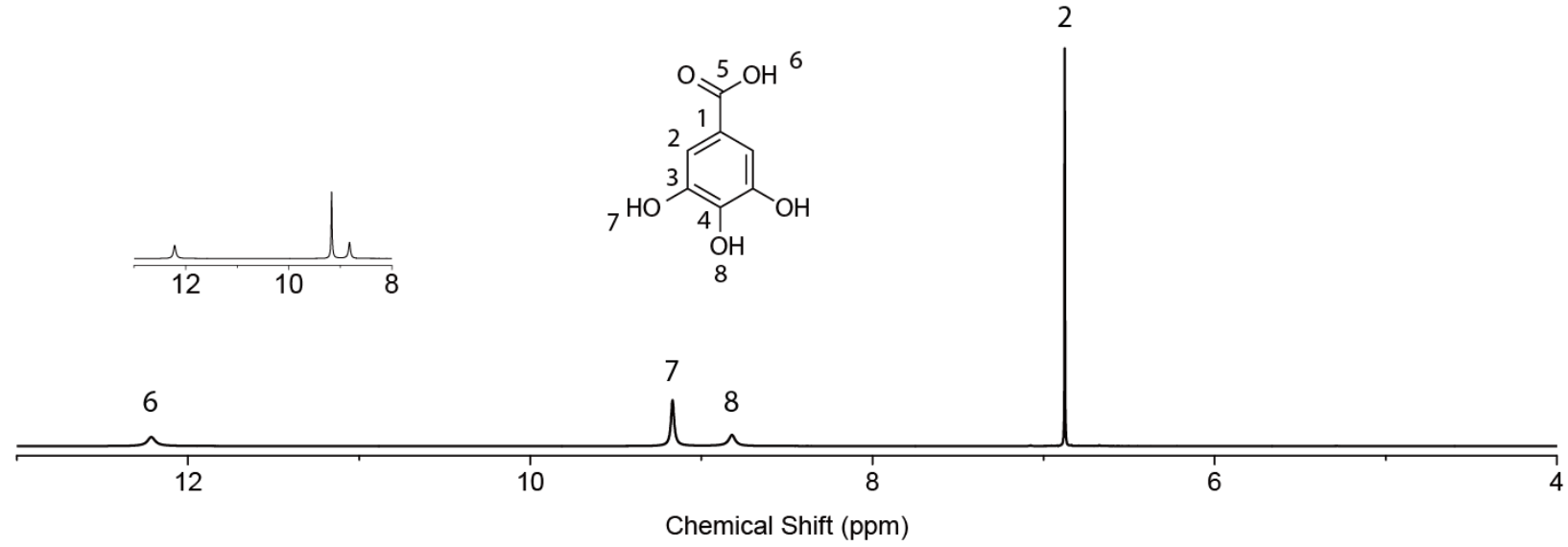

Figure S5a: Chemical structure of and ${ }^{1} \mathrm{H}$ NMR data obtained from gallic acid (referred to as "GalA" in main text).

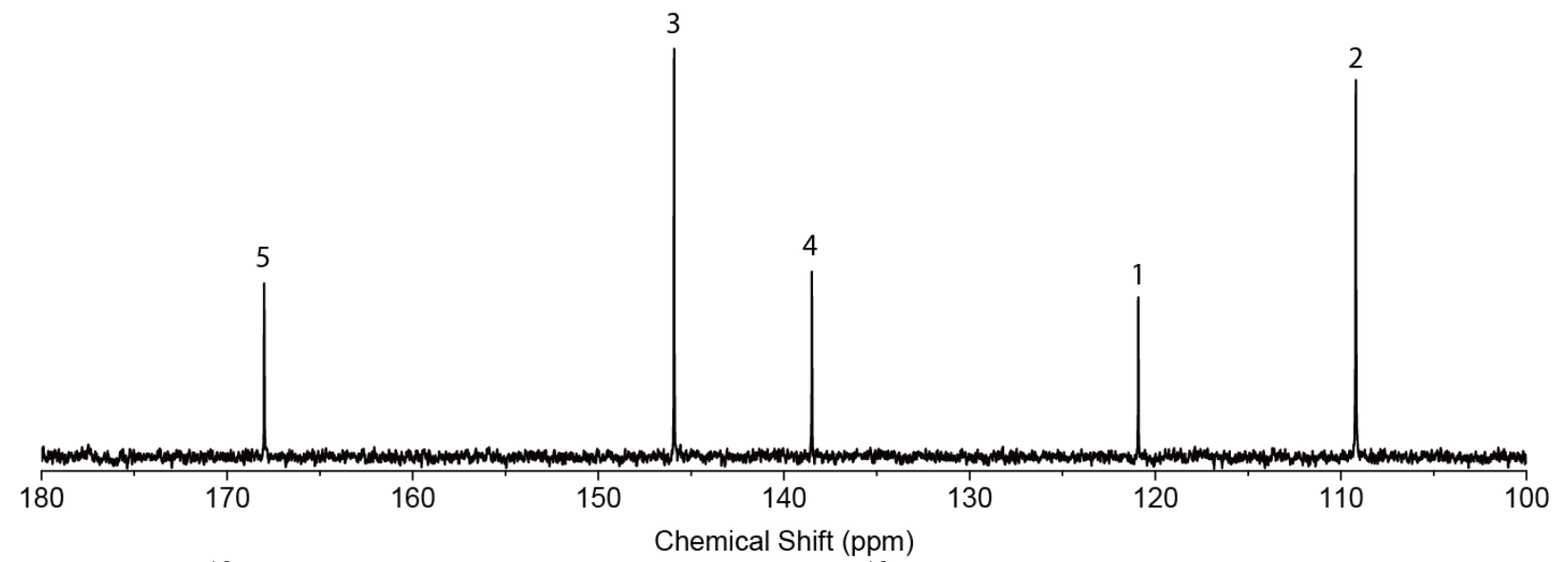

Figure S5b: ${ }^{13} \mathrm{C}$ NMR data obtained from gallic acid. ${ }^{13} \mathrm{C}$ NMR (100 MHz; DMSO-d 6 , ppm): $\delta$ $168.0,145.9,138.5,120.9,109.2$. 


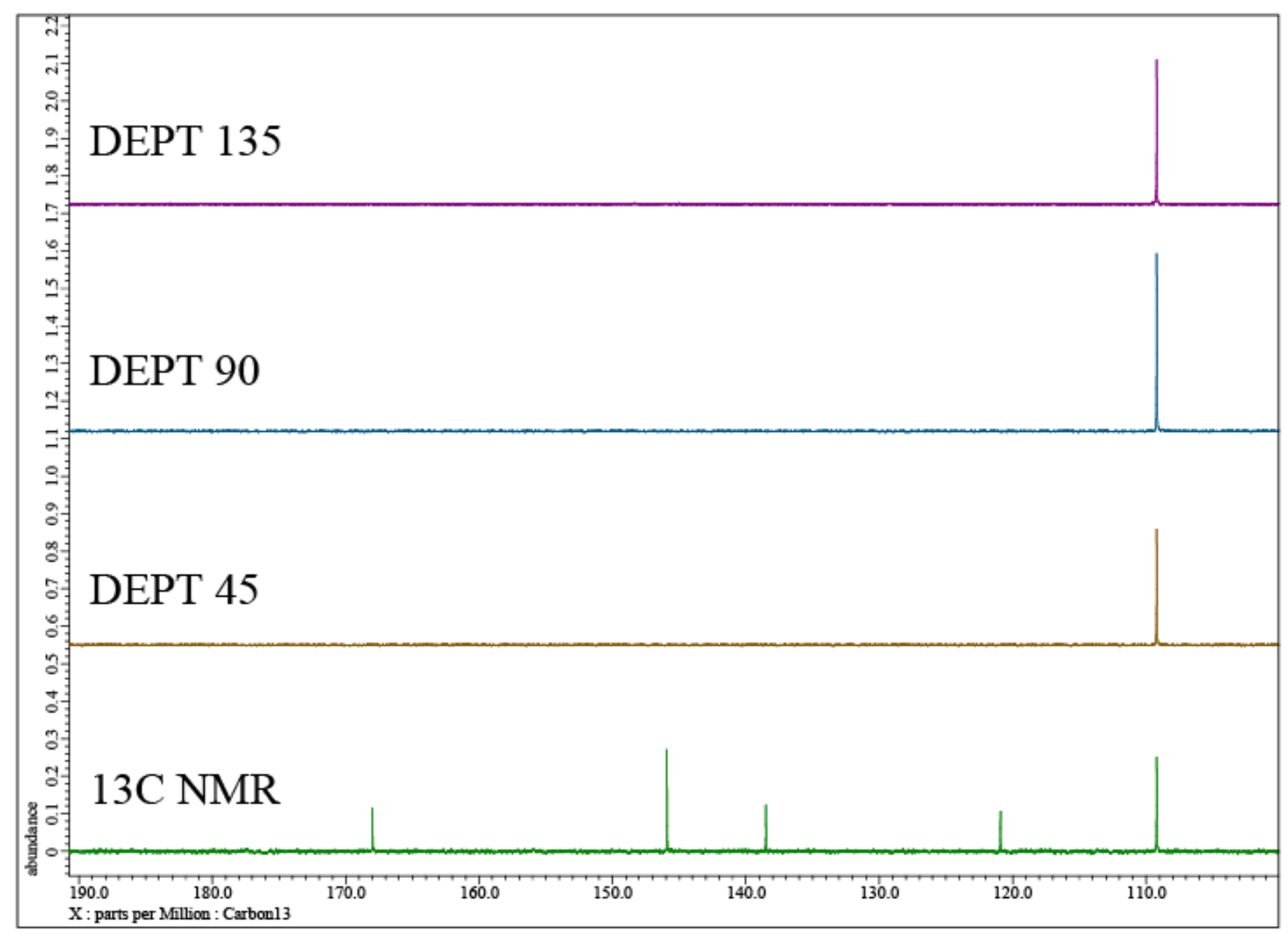

Figure S5c: ${ }^{13} \mathrm{C}$ NMR, DEPT 45, 90, 135 data obtained from gallic acid. 


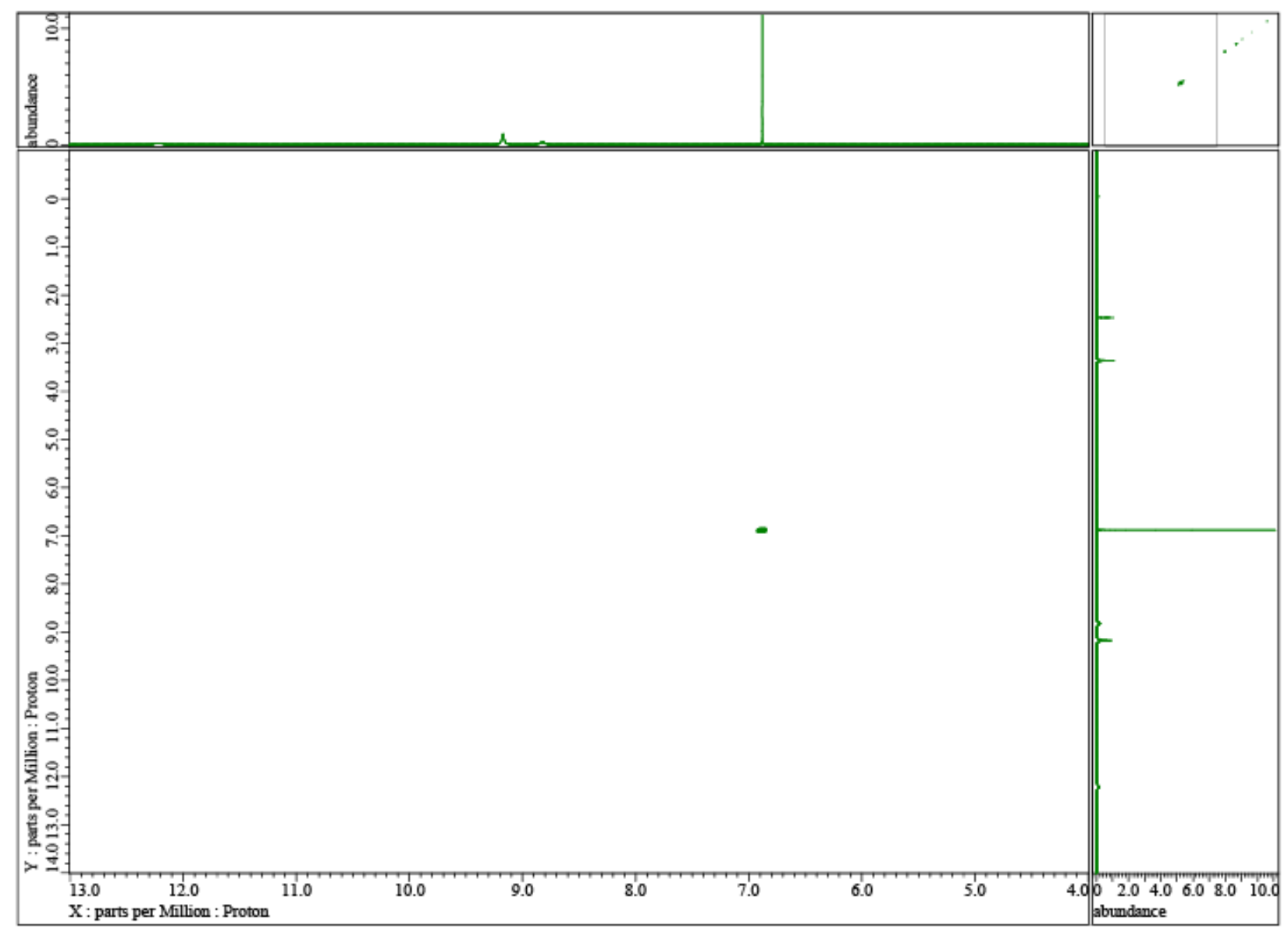

Figure S5d: COSY data obtained from gallic acid. 


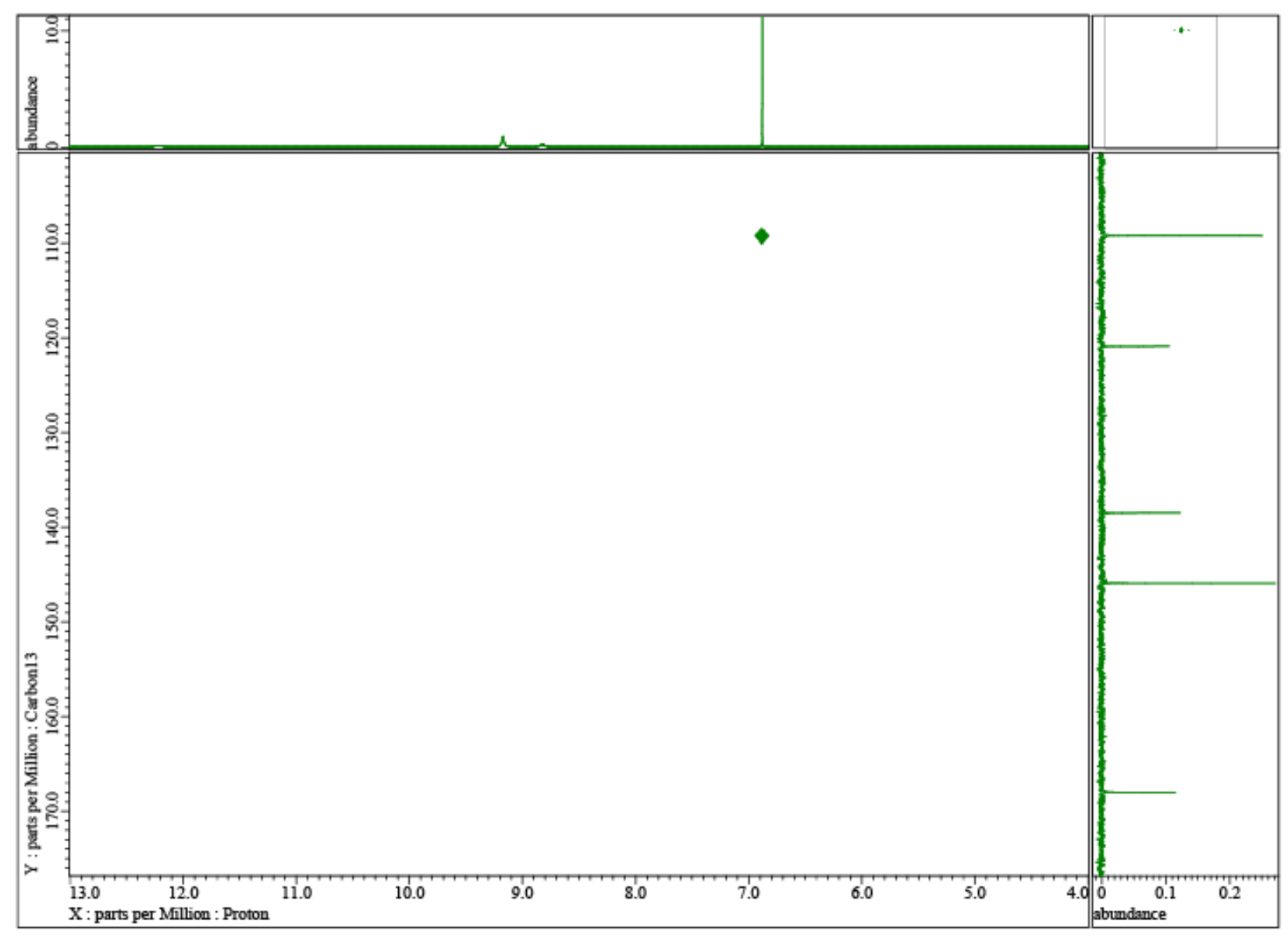

Figure S5e: HSQC data obtained from gallic acid. 


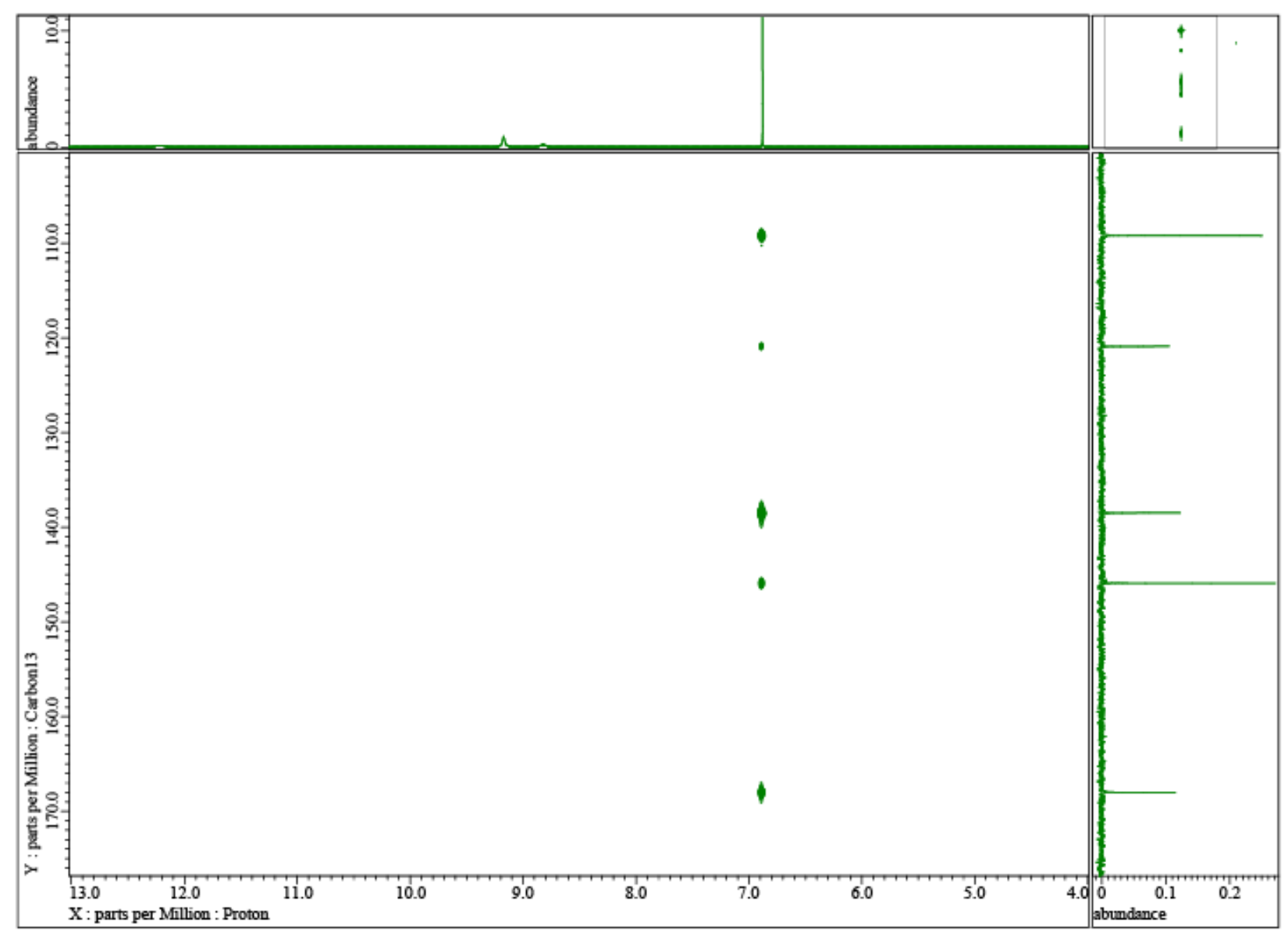

Figure S5f: HMBC data obtained from gallic acid. 


\section{Section 6: NMR Characterization of Allylated Gallic Acid (aGalA)}

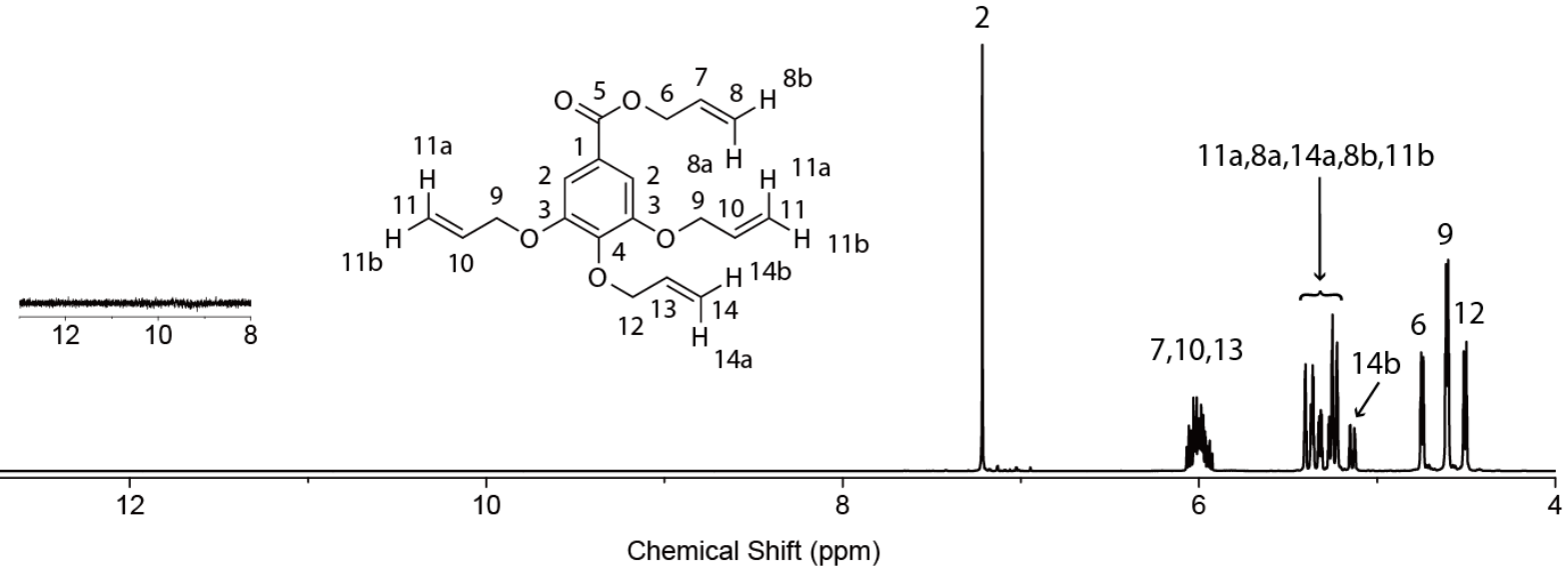

Figure S6a: Chemical structure of and ${ }^{1} \mathrm{H}$ NMR data obtained from allyl 3,4,5tris(allyloxy)benzoate (referred to as "allylated GalA" in main text). Data were obtained from final purified product. ): ${ }^{1} \mathrm{H}$ NMR (400 MHz, DMSO-d 6 , ppm): $\delta 7.21(\mathrm{~s}, 2 \mathrm{H}), 6.07-5.93(\mathrm{~m}, 4 \mathrm{H})$, $5.41-5.22(\mathrm{~m}, 7 \mathrm{H}), 5.14$ (ddt, $J=10.5,1.47,1.47 \mathrm{~Hz}, 1 \mathrm{H}), 4.75$ (ddd, $J=5.37,1.47,1.47 \mathrm{~Hz}, 2 \mathrm{H}$ ), 4.61 (ddd, $J=4.88,1.47,1.47 \mathrm{~Hz}, 4 \mathrm{H}), 4.51$ (ddd, $J=5.86,1.47,1.47 \mathrm{~Hz}, 2 \mathrm{H}$ ).
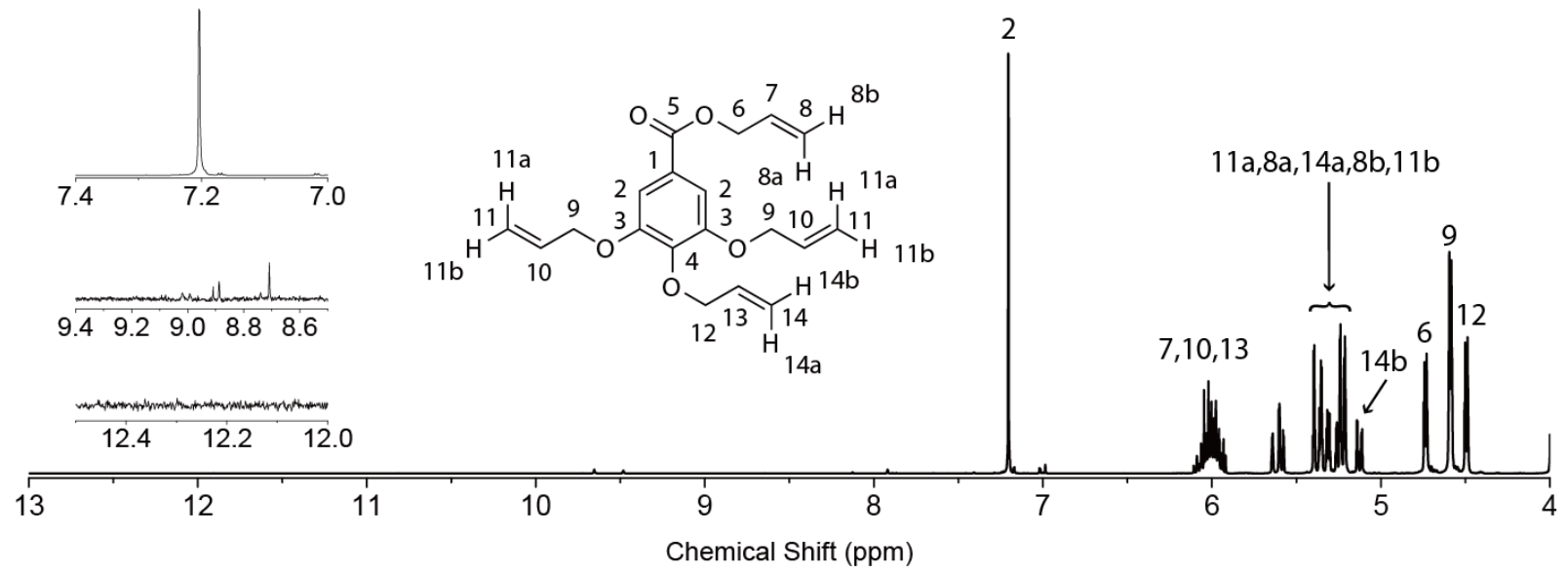

Figure S6b: Chemical structure of and ${ }^{1} \mathrm{H}$ NMR data obtained from allyl 3,4,5tris(allyloxy)benzoate. Data were obtained prior to extraction. Refer to Figure S3d for a closer view. 


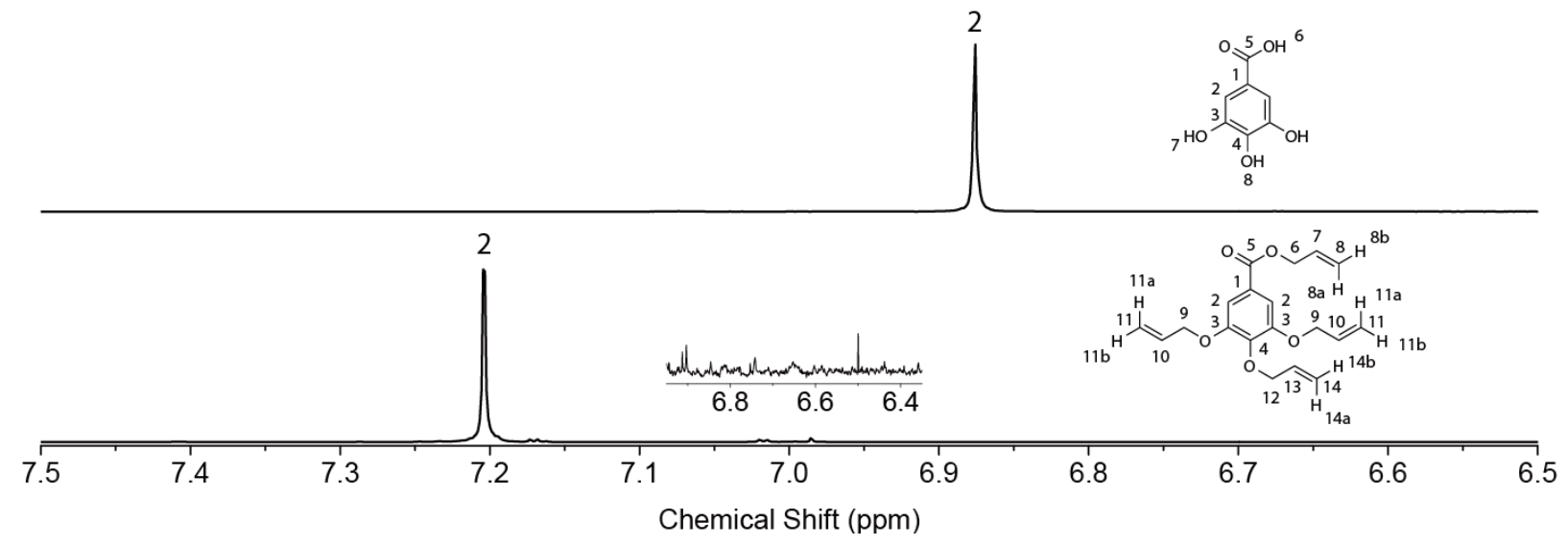

Figure S6c: ${ }^{1} \mathrm{H}$ NMR data obtained from (top) gallic acid and (bottom) allyl 3,4,5tris(allyloxy)benzoate. The spectrum of allyl 3,4,5-tris(allyloxy)benzoate was obtained prior to extraction for the purposes of determining the reaction conversion. Using the peak integrals for protons 2 , the reaction conversion was determined to be $100 \%$.

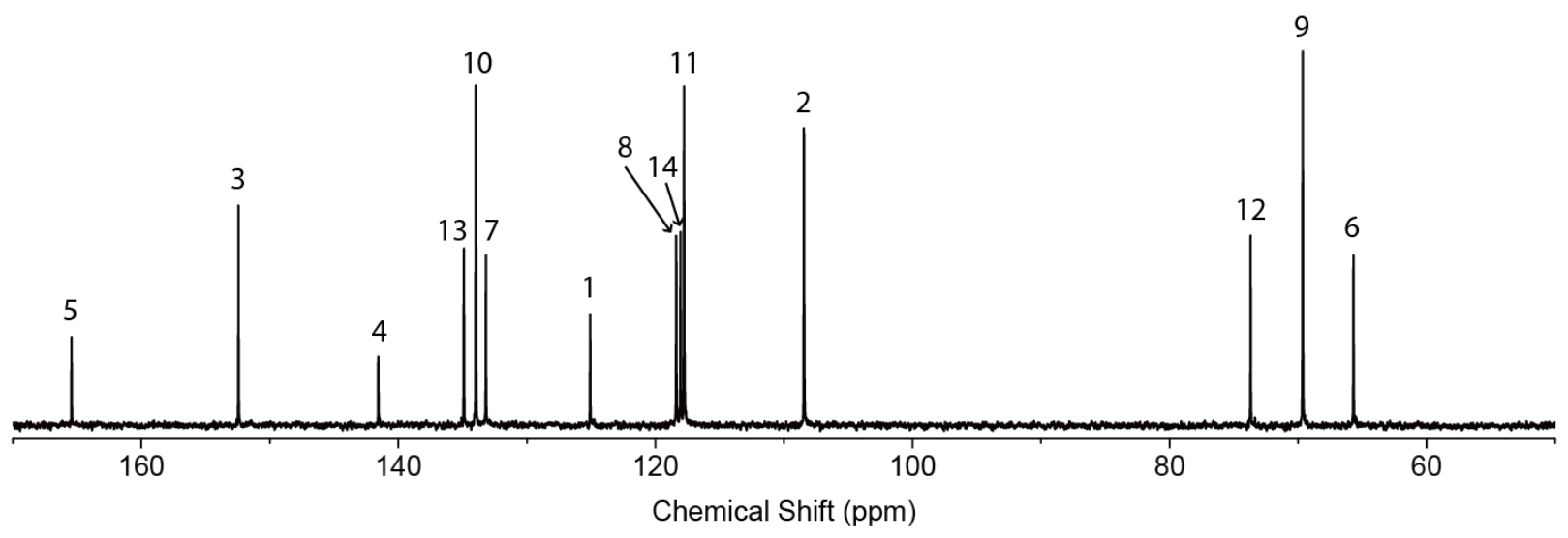

Figure S6d: ${ }^{13} \mathrm{C}$ NMR data obtained from allyl 3,4,5-tris(allyloxy)benzoate. 13C NMR (100 MHz; DMSO-d6): $\delta$ 165.4, 152.4, 141.6, 134.9, 134.0, 133.2, 125.1, 118.4, 118.0, 117.8, 108.4, 73.7, 69.9, 65.7. 


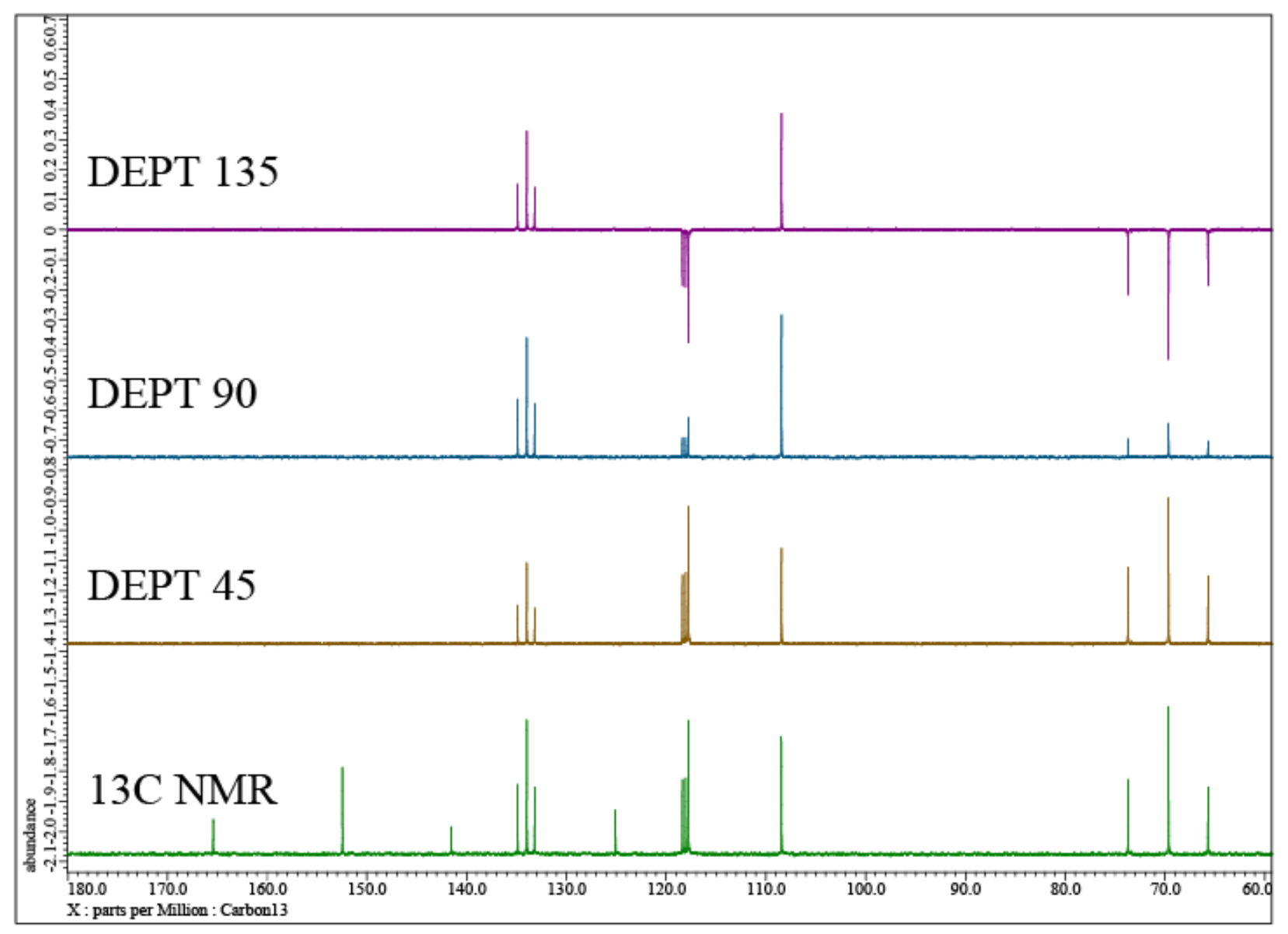

Figure S6e: ${ }^{13} \mathrm{C}$ NMR, DEPT 45, 90, 135 data obtained from allyl 3,4,5-tris(allyloxy)benzoate. 


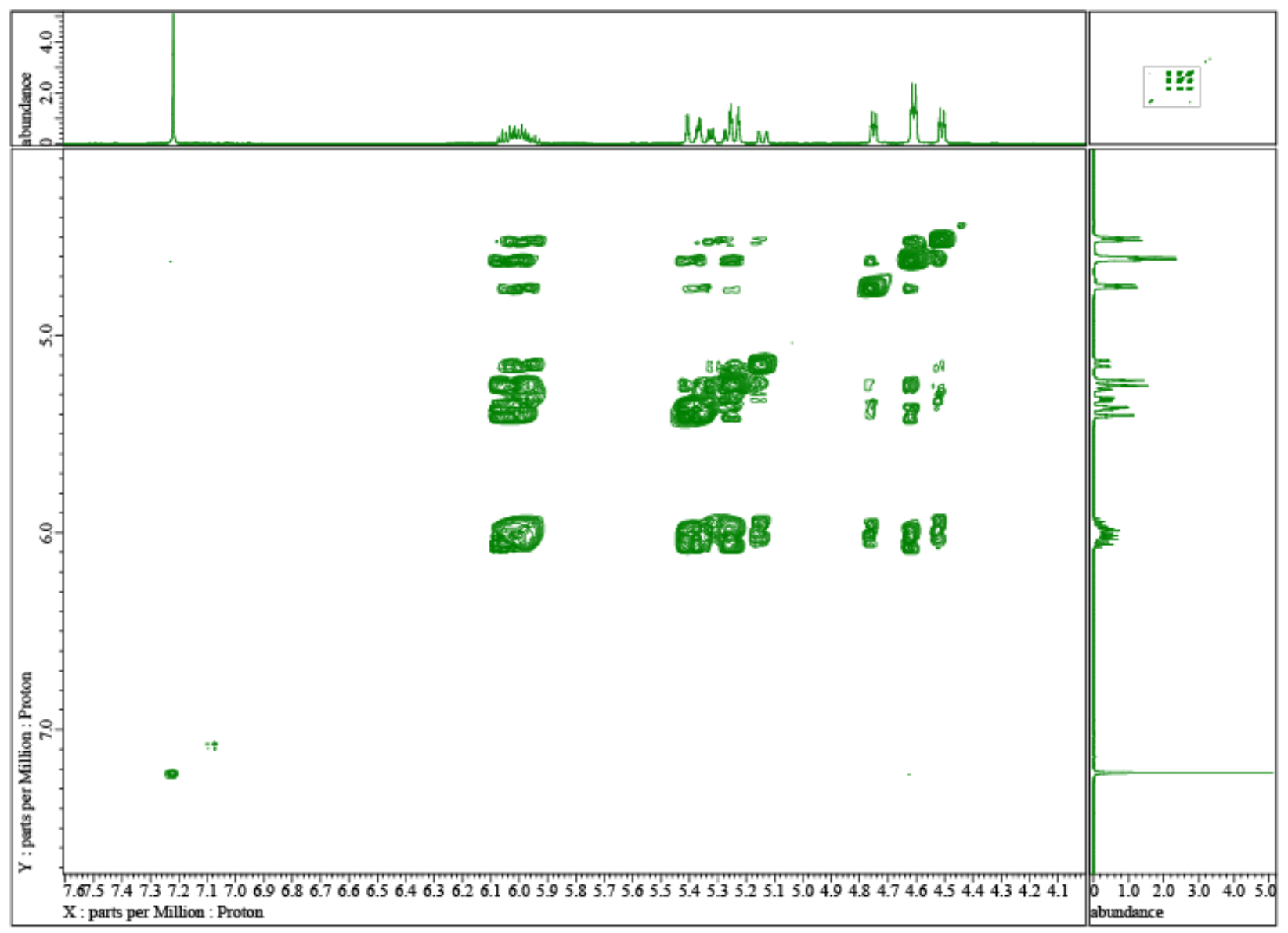

Figure S6f: COSY data obtained from allyl 3,4,5-tris(allyloxy)benzoate. 


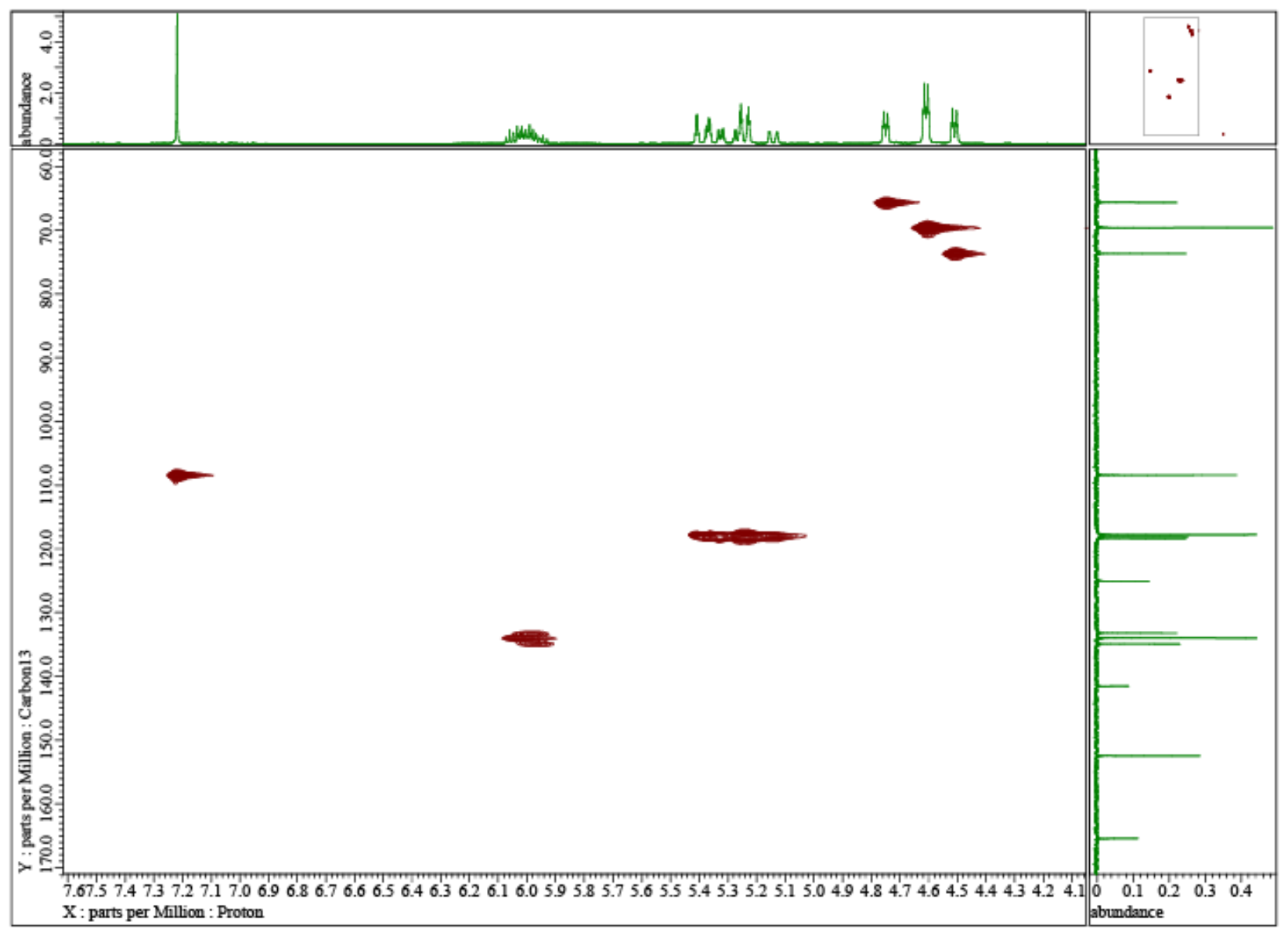

Figure S6g: HSQC data obtained from allyl 3,4,5-tris(allyloxy)benzoate. 


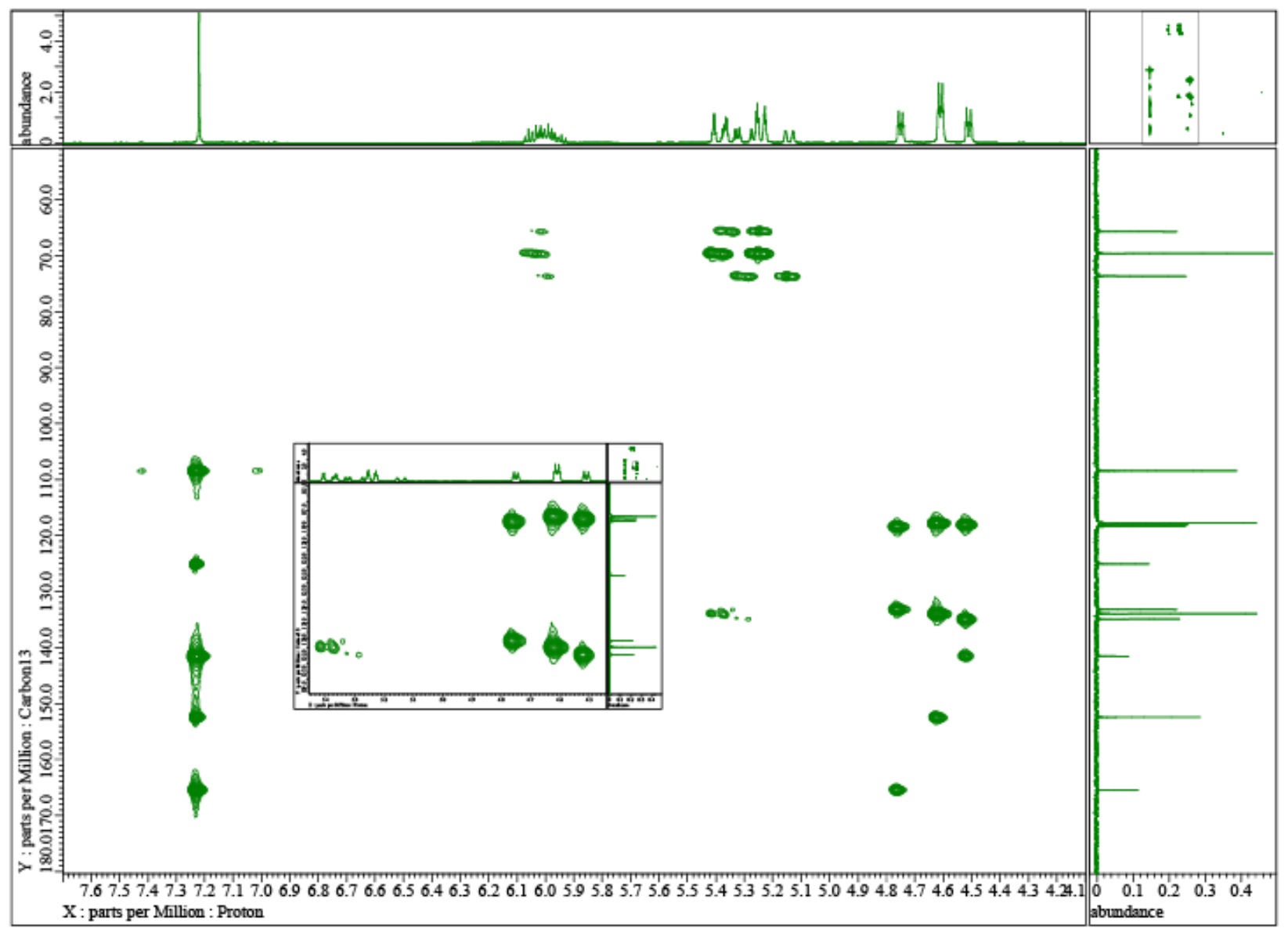

Figure S6h: HMBC data obtained from allyl 3,4,5-tris(allyloxy)benzoate. 


\section{Section 7: FTIR and DSC Characterization of Thiol-Ene Network Formation}

a)<smiles>[3H]C=CCOc1ccccc1C(=O)OCC=C</smiles>

b)<smiles>C=CCOC(=O)c1cccc(OCC=C)c1</smiles>

c)<smiles>C=CCOC(=O)c1ccc(OCC=C)cc1</smiles>
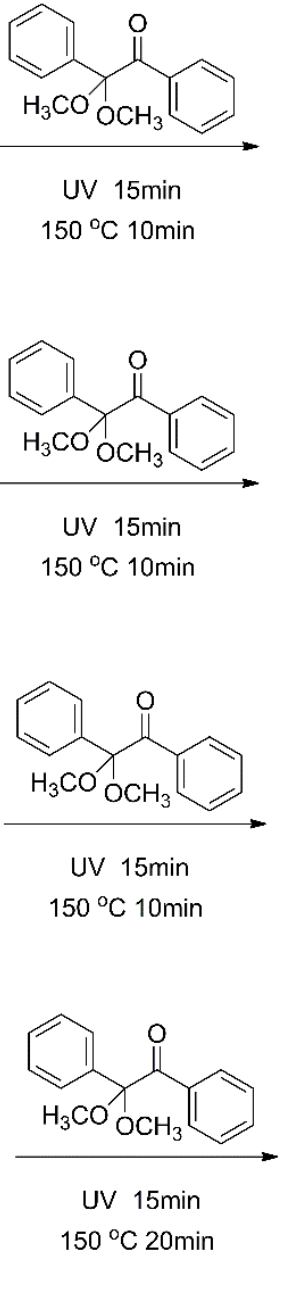<smiles>C=CCOC(=O)c1cc(OCC=C)ccc1OCC=C</smiles><smiles>O=C(CCS)OCC(COC(=O)CCS)(COC(=O)CCS)COC(=O)CCS</smiles><smiles>C=CCOC(=O)c1cc(OCC=C)c(OCC=C)c(OCC=C)c1</smiles><smiles>O=C(CCS)OCC(COC(=O)CCS)(COC(=O)CCS)COC(=O)CCS</smiles>

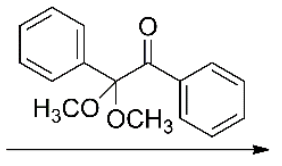

UV $15 \mathrm{~min}$ $150^{\circ} \mathrm{C} 20 \mathrm{~min}$

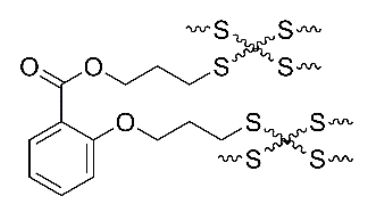

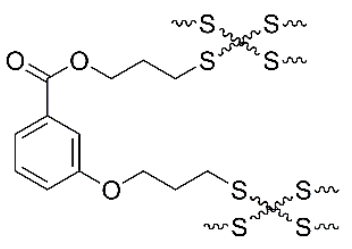

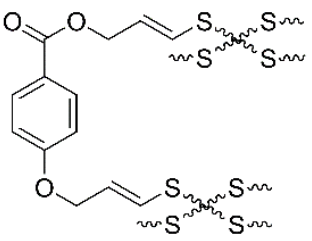

Scheme S1: Photoinitated thiol-ene reaction between the tetra-functional thiol PETMP and a) aSA, b) a3HBA, c) a4HBA, d) aGenA and e) aGalA. 


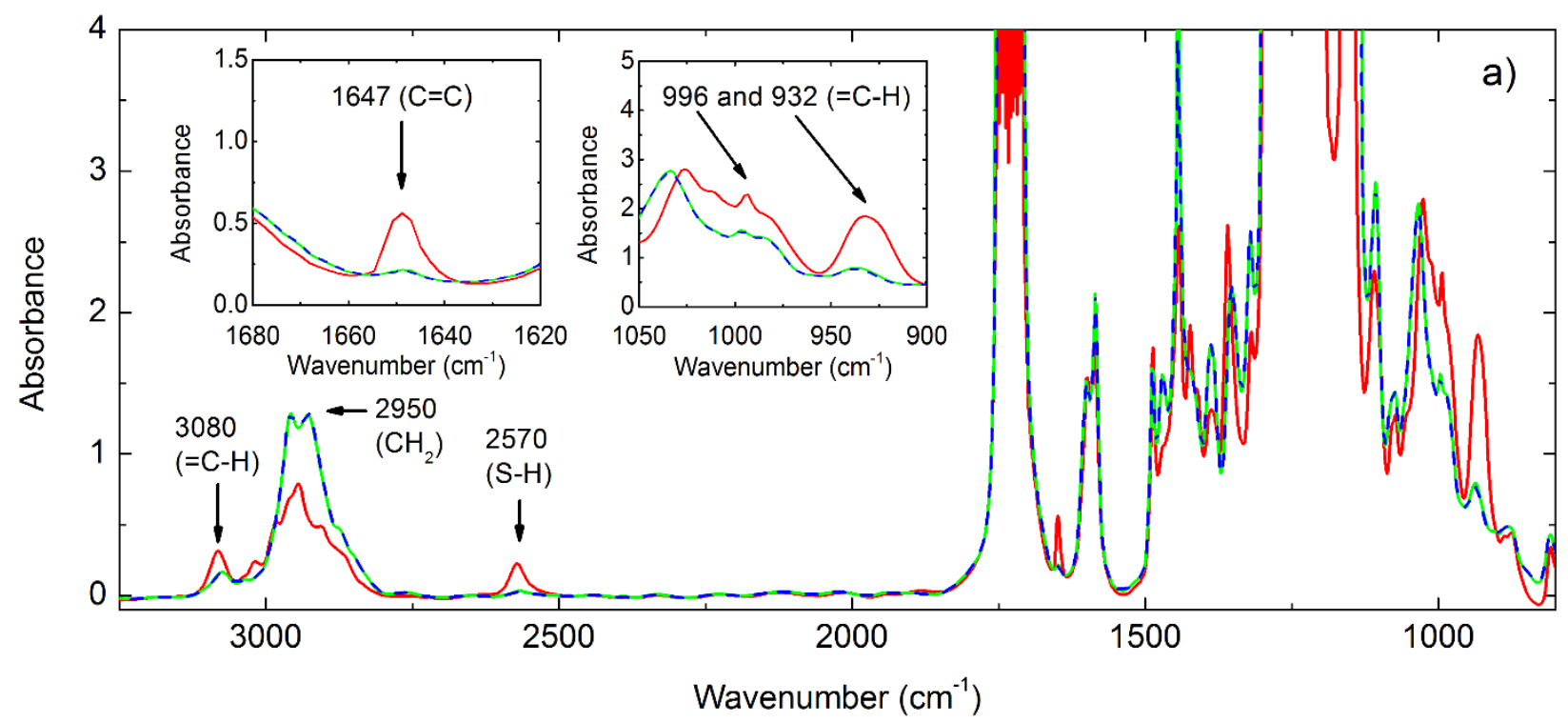

Figure S7a: FTIR data obtained from a mixture of PETMP, photoinitiator, and a3HBA: before curing (red solid curve), after $15 \mathrm{~min}$ of UV exposure (green solid curve), and after $30 \mathrm{~min}$ of UV exposure (dark blue dashed curve). The following peaks are highlighted: 932 and $996 \mathrm{~cm}^{-1}$ (olefinic $=\mathrm{C}-\mathrm{H}$ bending, allyl group), $1647 \mathrm{~cm}^{-1}\left(\mathrm{C}=\mathrm{C}\right.$ stretching, allyl group), $2570 \mathrm{~cm}^{-1}(\mathrm{~S}-\mathrm{H}$ stretching, PETMP), $2950 \mathrm{~cm}^{-1}$ (alkane C-H stretching, polymer network), and $3080 \mathrm{~cm}^{-1}$ (olefinic $=\mathrm{C}-\mathrm{H}$ stretching, allyl group). 

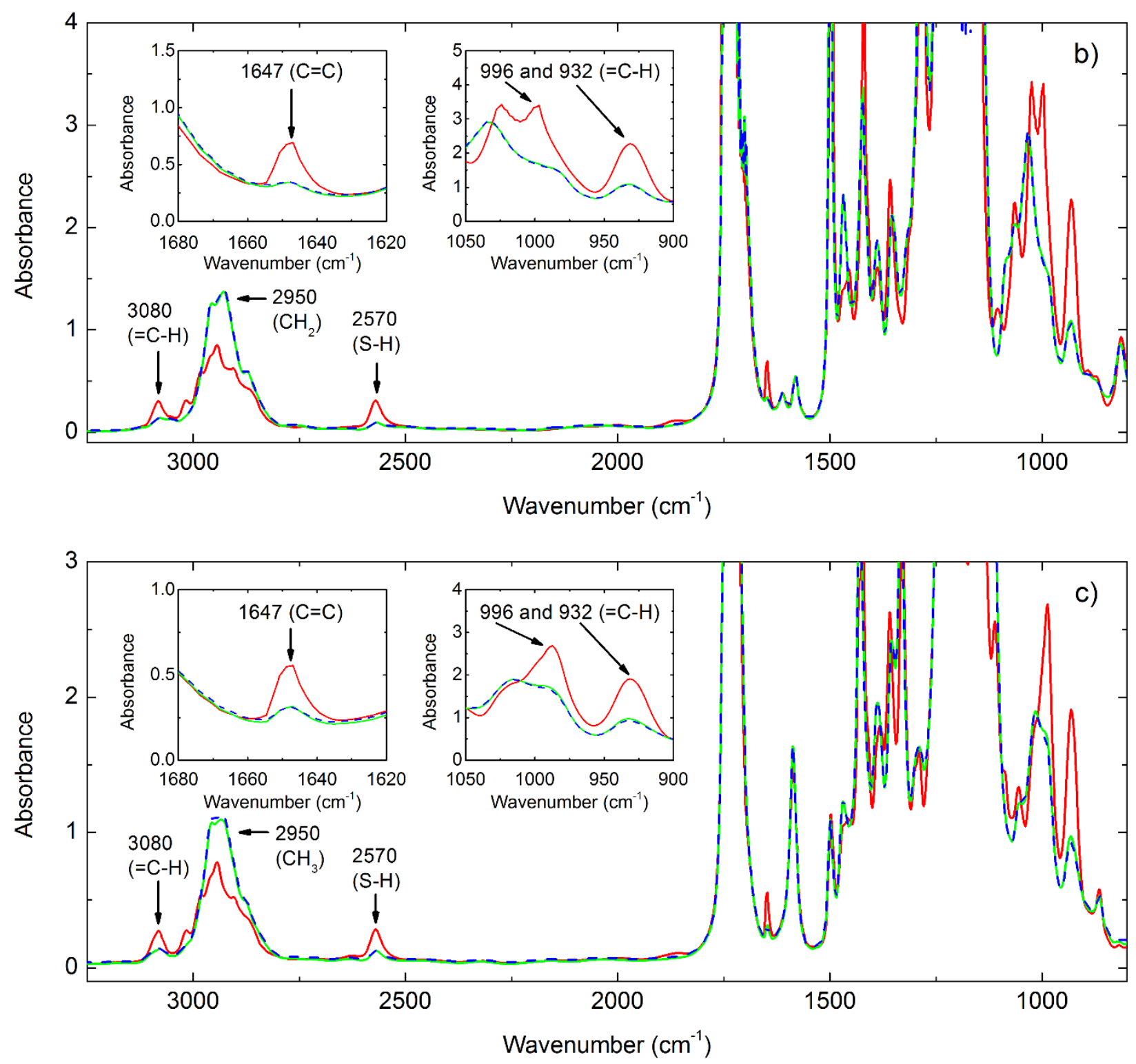

Figure S7b and S7c: FTIR data obtained from a mixture of PETMP, photoinitiator, and (b) aGenA or (c) aGalA: before curing (red solid curve), after $15 \mathrm{~min}$ of UV exposure (green solid curve), and after 30 min of UV exposure (dark blud dashed curve). The following peaks are highlighted: 932 and $996 \mathrm{~cm}^{-1}$ (olefinic $=\mathrm{C}-\mathrm{H}$ bending, allyl group), $1647 \mathrm{~cm}^{-1}(\mathrm{C}=\mathrm{C}$ stretching, allyl group), 2570 $\mathrm{cm}^{-1}$ (S-H stretching, PETMP), $2950 \mathrm{~cm}^{-1}$ (alkane C-H stretching, polymer network), and 3080 $\mathrm{cm}^{-1}$ (olefinic $=\mathrm{C}-\mathrm{H}$ stretching, allyl group). 

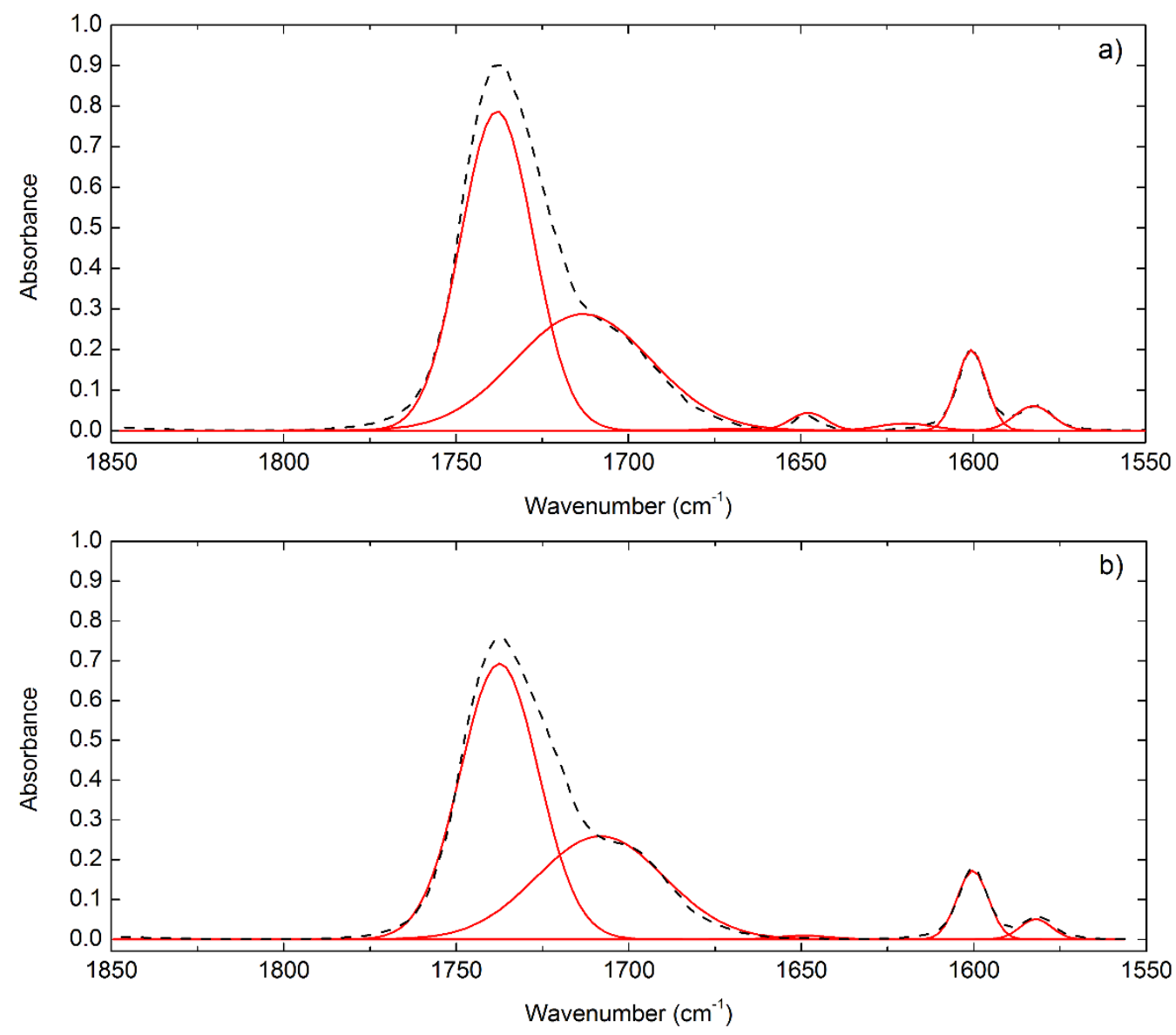

Figure S7d: FTIR data obtained on aSA-based thiol-ene network: a) prior to curing and b) following 15 min of UV curing.

The data shown in the Figures S7a-c were taken on a $0.05 \mathrm{~mm}$ thick specimen, which was thick enough that the larger peaks exhibited very high absorbances. However, this larger thickness allowed the relevant peaks to be more visible (i.e. peaks for $\mathrm{C}=\mathrm{C}$ and $=\mathrm{C}-\mathrm{H}$ labeled in Figures S7a-c). A thinner film was prepared (without a spacer), which allowed the neighboring peaks to be fully observed (data shown here). We attempted to deconvolve the peaks, but the peaks associated with the allyl group were too small and the resulting analysis was not meaningful to quantify the conversion of allyl groups. For example, prior to UV curing [plot a)], we observed the $\mathrm{C}=\mathrm{C}$ stretching peak at $1647 \mathrm{~cm}^{-1}$, however, following 15 min of UV curing [plot b)], this peak was not resolved even with deconvolution. In a) and b), the black dashed curves are the data and red solid curves are the deconvolutions. 
Table S1: Assignment of FTIR Vibrational Modes

\begin{tabular}{|l|l|l|}
\hline $\begin{array}{l}\text { Wavenumber at FTIR peak } \\
\text { maximum }\left(\mathrm{cm}^{-1}\right)\end{array}$ & Vibrational mode & References \\
\hline 932 & olefinic $=\mathrm{C}-\mathrm{H}$ bending & $1-6$ \\
\hline 996 & olefinic $=\mathrm{C}-\mathrm{H}$ bending & $1-6$ \\
\hline 1647 & C=C stretching & $3,5-13$ \\
\hline 2570 & S-H stretching & $1-14$ \\
\hline 2950 & alkane C-H stretching & 6 \\
\hline 3080 & olefinic $=\mathrm{C}-\mathrm{H}$ stretching & $4,6,8,10,12$ \\
\hline
\end{tabular}


Conversion was quantified through measurement of the maximum intensity of the absorbance peak associated with S-H stretching $\left(2570 \mathrm{~cm}^{-1}\right)$. Quantifying the conversion from this FTIR data has significant uncertainty, due to the small size of this peak (note the high absorbance of other peaks that were not relevant to the thiol-ene reaction, due to the large sample thickness required for this measurement). The error on measurements described in the table below represent multiple measurements obtained on independently prepared specimens.

\section{Table S2: Conversion of aSA Network}

Measurements on multiple specimens:

\begin{tabular}{|c|c|}
\hline Reaction Condition & Conversion \\
\hline $15 \min \mathrm{UV}$ & $93 \% \pm 0.4 \%$ \\
\hline $15 \min \mathrm{UV}+10 \min 150^{\circ} \mathrm{C}$ & $97 \% \pm 0.1 \%$ \\
\hline
\end{tabular}

Measurement on a single specimen to probe influence of UV curing time:

\begin{tabular}{|c|c|}
\hline Reaction Condition & Conversion \\
\hline 15 min UV & $90 \%$ \\
\hline 30 min UV & $90 \%$ \\
\hline
\end{tabular}

Table S3: Conversion of a3HBA Network

Measurements on multiple specimens:

\begin{tabular}{|c|c|}
\hline Reaction Condition & Conversion \\
\hline $15 \min \mathrm{UV}$ & $80 \% \pm 4 \%$ \\
\hline $15 \min \mathrm{UV}+10 \min 150^{\circ} \mathrm{C}$ & $88 \% \pm 3 \%$ \\
\hline
\end{tabular}

Measurement on a single specimen to probe influence of UV curing time:

\begin{tabular}{|c|c|}
\hline Reaction Condition & Conversion \\
\hline $15 \mathrm{~min}$ UV & $82 \%$ \\
\hline $30 \mathrm{~min}$ UV & $83 \%$ \\
\hline
\end{tabular}

Table S4: Conversion of a4HBA Network

Measurements on multiple specimens:

\begin{tabular}{|c|c|}
\hline Reaction Condition & Conversion \\
\hline $15 \min \mathrm{UV}$ & $82 \% \pm 1 \%$ \\
\hline $15 \min \mathrm{UV}+10 \min 150^{\circ} \mathrm{C}$ & $88 \% \pm 2 \%$ \\
\hline
\end{tabular}

Measurement on a single specimen to probe influence of UV curing time:

\begin{tabular}{|c|c|}
\hline Reaction Condition & Conversion \\
\hline 15 min UV & $86 \%$ \\
\hline 30 min UV & $86 \%$ \\
\hline
\end{tabular}




\section{Table S5: Conversion of aGenA Network}

Measurements on multiple specimens:

\begin{tabular}{|c|c|}
\hline Reaction Condition & Conversion \\
\hline $15 \min U V$ & $76 \% \pm 1 \%$ \\
\hline $15 \min \mathrm{UV}+20 \mathrm{~min} 150{ }^{\circ} \mathrm{C}$ & $88 \% \pm 2 \%$ \\
\hline
\end{tabular}

Measurement on a single specimen to probe influence of UV curing time:

\begin{tabular}{|c|c|}
\hline Reaction Condition & Conversion \\
\hline $15 \mathrm{~min} U V$ & $78 \%$ \\
\hline $30 \mathrm{~min} \mathrm{UV}$ & $80 \%$ \\
\hline
\end{tabular}

Table S6: Conversion of aGalA Network

Measurements on multiple specimens:

\begin{tabular}{|c|c|}
\hline Reaction Condition & Conversion \\
\hline $15 \min U V$ & $71 \% \pm 2 \%$ \\
\hline $15 \min \mathrm{UV}+20 \min 150{ }^{\circ} \mathrm{C}$ & $83 \% \pm 2 \%$ \\
\hline
\end{tabular}

Measurement on a single specimen to probe influence of UV curing time:

\begin{tabular}{|c|c|}
\hline Reaction Condition & Conversion \\
\hline $15 \mathrm{~min} U V$ & $67 \%$ \\
\hline $30 \mathrm{~min}$ UV & $71 \%$ \\
\hline
\end{tabular}

Table S7: Summary of Final Conversions for Curing Protocol Used in Manuscript

\begin{tabular}{|c|c|c|}
\hline Allylated Phenolic Acid & Reaction Condition & Conversion \\
\hline aSA & $15 \min U V+10 \min 150{ }^{\circ} \mathrm{C}$ & $97 \% \pm 0.1 \%$ \\
\hline a3HBA & $15 \min \mathrm{UV}+10 \min 150^{\circ} \mathrm{C}$ & $88 \% \pm 3 \%$ \\
\hline a4HBA & $15 \min \mathrm{UV}+10 \min 150^{\circ} \mathrm{C}$ & $88 \% \pm 2 \%$ \\
\hline $\mathrm{aGenA}$ & $15 \min \mathrm{UV}+20 \min 150^{\circ} \mathrm{C}$ & $88 \% \pm 2 \%$ \\
\hline $\mathrm{aGalA}$ & $15 \min \mathrm{UV}+20 \min 150^{\circ} \mathrm{C}$ & $83 \% \pm 2 \%$ \\
\hline
\end{tabular}



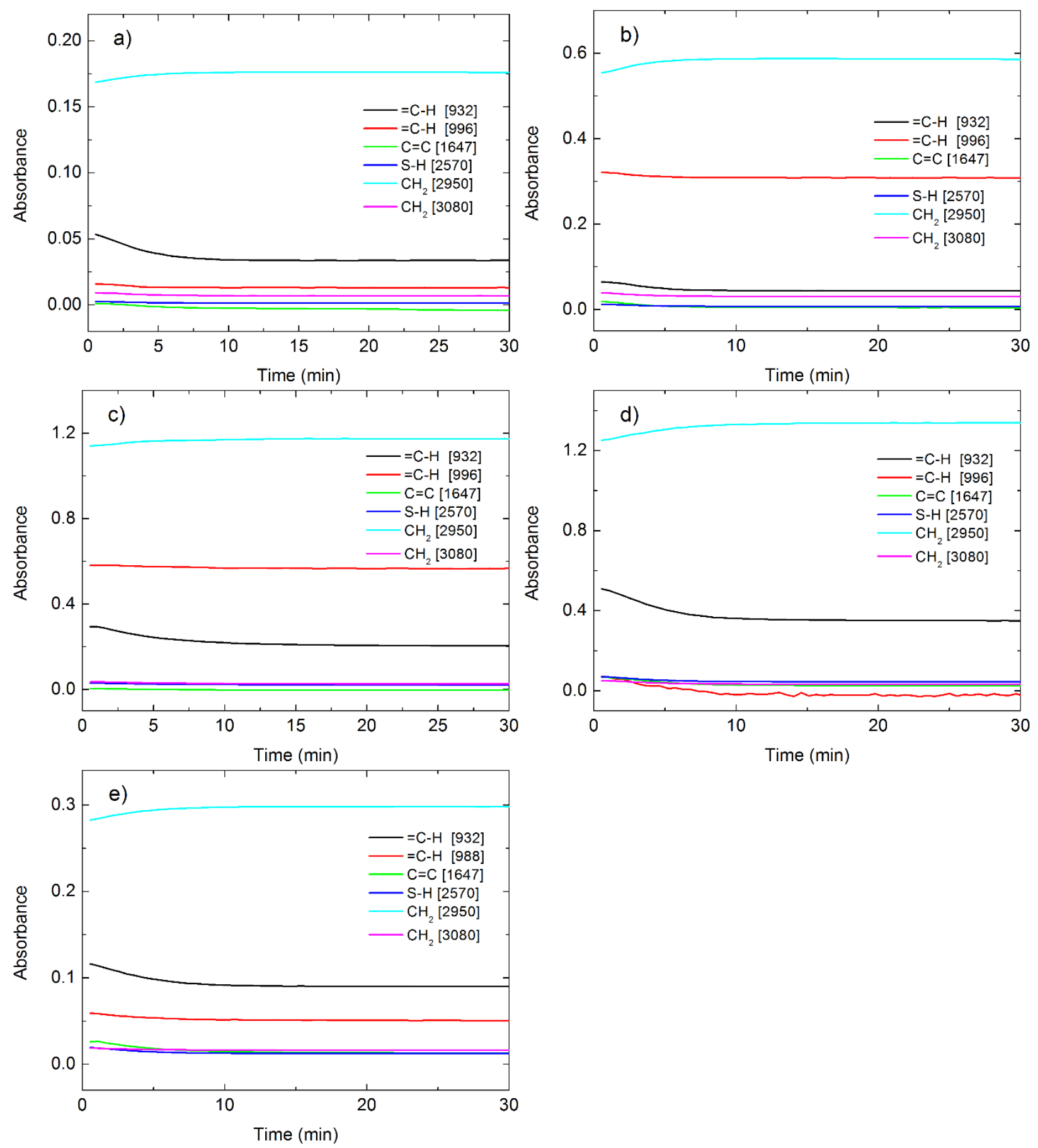

Figure S8: The FTIR absorbance as a function of time for selected peaks while curing at $150{ }^{\circ} \mathrm{C}$ (following 15 min of UV exposure) is shown in thiol-ene network derived from a) aSA, b) a3HBA, c) a4HBA, d) aGenA and e) aGalA. 

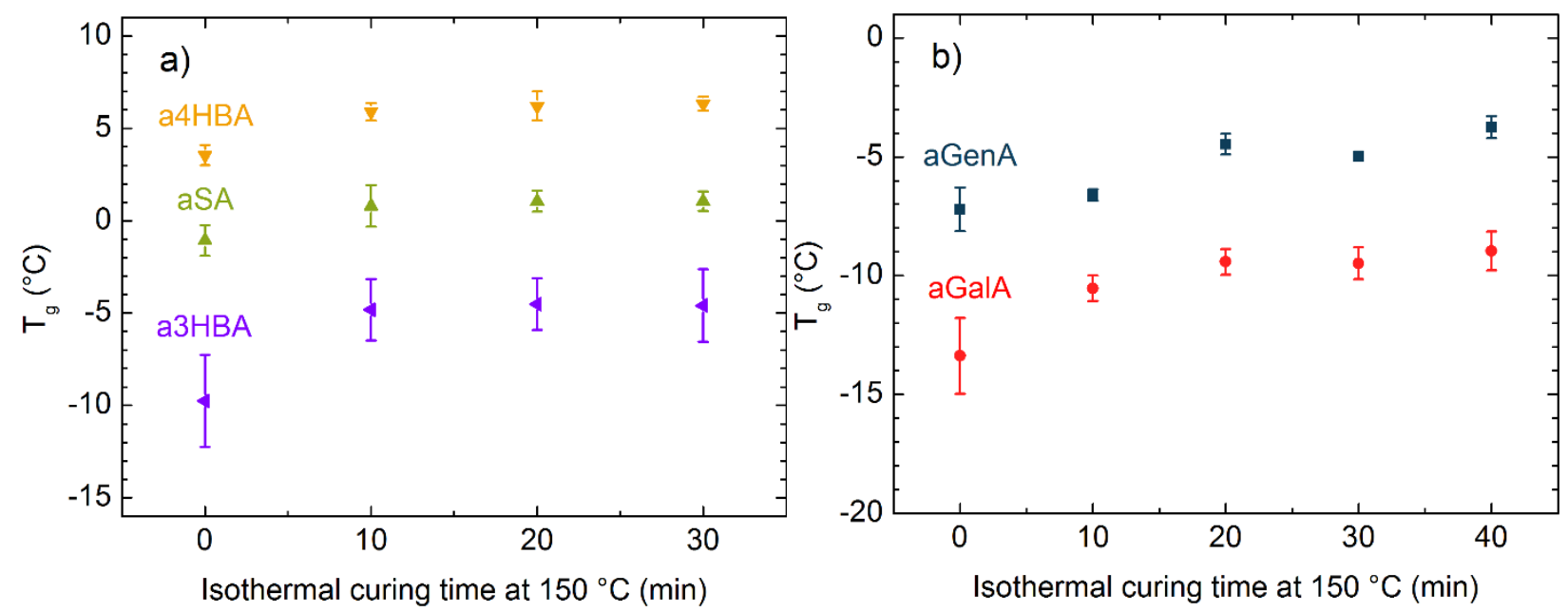

Figure S9 Glass transition temperature $\left(\mathrm{T}_{\mathrm{g}}\right)$ as a function of isothermal curing time at $150{ }^{\circ} \mathrm{C}$ (following 15 min of exposure to UV) for thiol-ene networks derived from (a) aSA (green $\mathbf{\Delta}$ ),

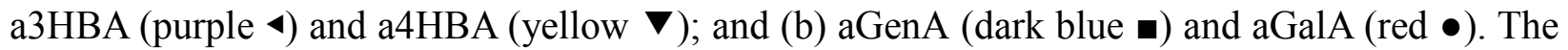
data points shown were obtained from four distinct regions of the same specimen (which were isothermally cured for different lengths of time). The results of the first heating scan are shown in this figure. Data shown for aSA and a4HBA were previously reported in ref. ${ }^{15}$. 


\section{Section 8: Physical Properties of Thiol-Ene Networks}
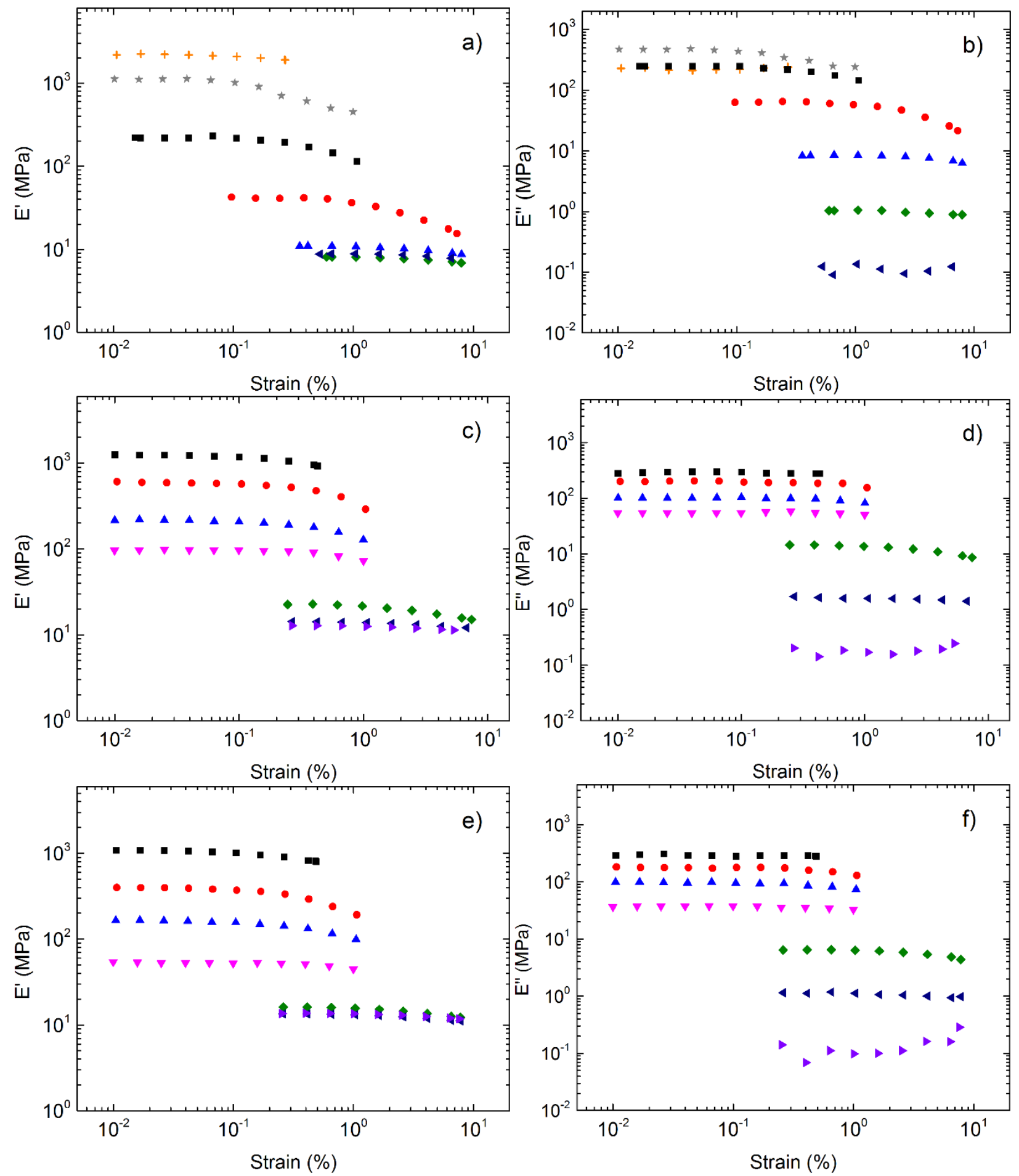

Figure S10: Storage (E') and loss (E') moduli as a function of strain (obtained from DMA, using a frequency of $1 \mathrm{~Hz}$ ) of thiol-ene networks derived from (a) (b) a3HBA, (c) (d) aGenA and (e) (f) aGalA. Data were obtained at the following temperatures: $-10^{\circ} \mathrm{C}(+),-5^{\circ} \mathrm{C}(\star), 0{ }^{\circ} \mathrm{C}(\boldsymbol{\bullet}), 5^{\circ} \mathrm{C}$ $(\bullet), 10{ }^{\circ} \mathrm{C}(\boldsymbol{\Delta}), 15^{\circ} \mathrm{C}(\nabla), 20^{\circ} \mathrm{C}(\bullet), 30{ }^{\circ} \mathrm{C}(\triangleleft), 40^{\circ} \mathrm{C}(\bullet)$. 

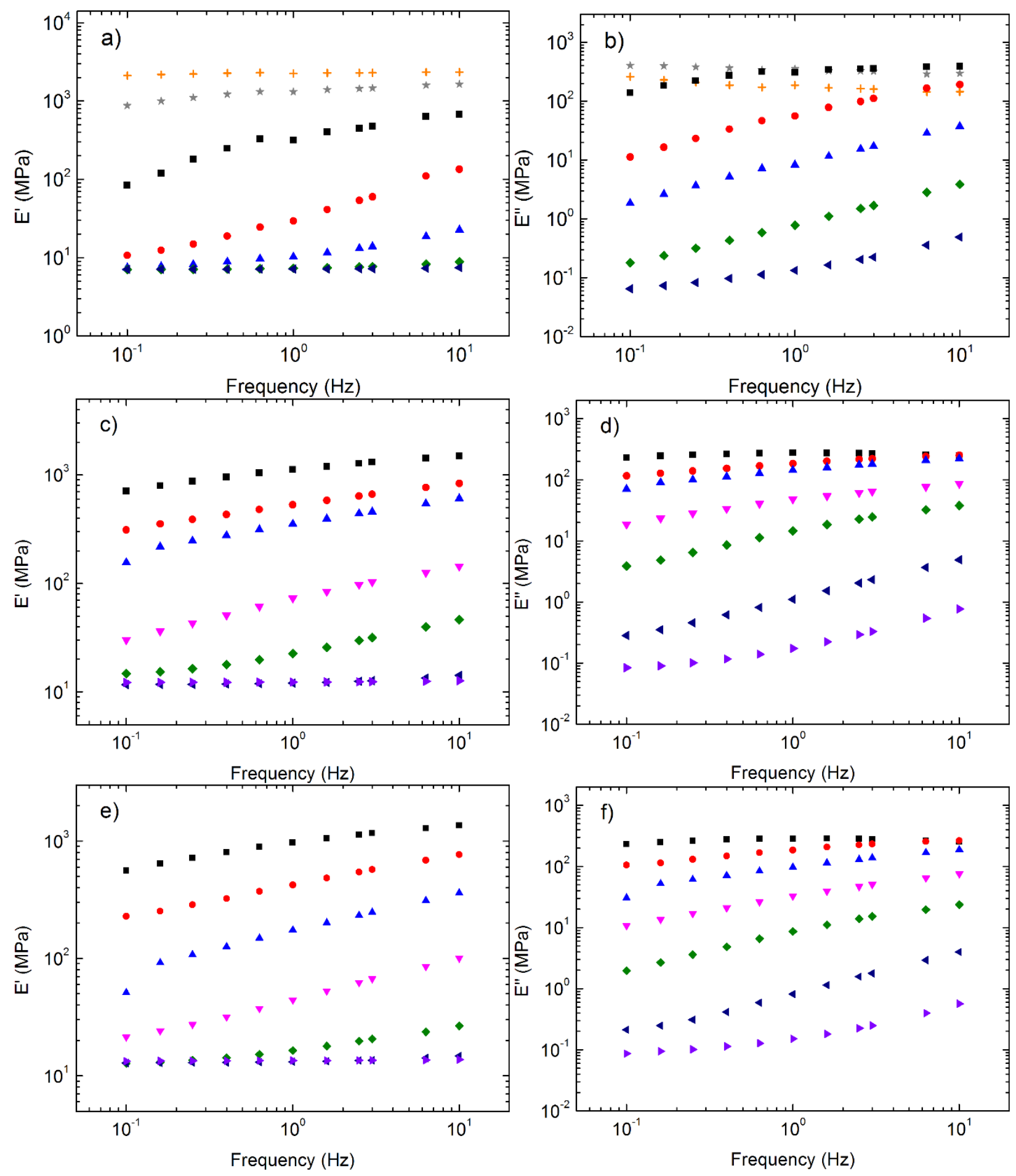

Figure S11: Storage (E') and loss (E') moduli as a function of frequency (obtained from DMA, using a strain value in the linear region) of thiol-ene networks derived from (a) (b) a3HBA, (c) (d) aGenA and (e) (f) aGalA. Data were obtained at the following temperatures: $-10^{\circ} \mathrm{C}(+),-5^{\circ} \mathrm{C}(\star)$, $0{ }^{\circ} \mathrm{C}(\boldsymbol{\bullet}), 5^{\circ} \mathrm{C}(\bullet), 10{ }^{\circ} \mathrm{C}(\boldsymbol{\Delta}), 15^{\circ} \mathrm{C}(\boldsymbol{\nabla}), 20{ }^{\circ} \mathrm{C}(\diamond), 30{ }^{\circ} \mathrm{C}(\triangleleft), 40{ }^{\circ} \mathrm{C}(\triangleright)$. 
Table S8: Crosslink Density of aSA Networks Determined through DMA

\begin{tabular}{|c|c|c|c|}
\hline Batch \# & Specimen \# & $\mathrm{E}_{\mathrm{r}}^{\prime}$ in rubbery plateau $(\mathrm{MPa})$ at $30^{\circ} \mathrm{C}$ & $v_{c}\left(\times 10^{-3} \mathrm{~mol} / \mathrm{cm}^{3}\right)$ \\
\hline $1^{\mathrm{a}}$ & 1 & 7.11 & 0.94 \\
\hline \multirow{3}{*}{2} & 1 & 8.83 & 1.17 \\
\hline & 2 & 8.73 & 1.15 \\
\hline & 3 & 8.65 & 1.14 \\
\hline \multicolumn{2}{|c|}{ Average of batch 2} & $8.74 \pm 0.09$ & $1.16 \pm 0.01$ \\
\hline \multirow{3}{*}{3} & 1 & 9.55 & 1.26 \\
\hline & 2 & 9.24 & 1.22 \\
\hline & 3 & 8.55 & 1.13 \\
\hline \multicolumn{2}{|c|}{ Average of batch 3} & $9.11 \pm 0.51$ & $1.21 \pm 0.07$ \\
\hline \multirow{3}{*}{4} & 1 & 8.76 & 1.16 \\
\hline & 2 & 9.24 & 1.22 \\
\hline & 3 & 8.63 & 1.14 \\
\hline \multicolumn{2}{|c|}{ Average of batch 4} & $8.88 \pm 0.32$ & $1.17 \pm 0.04$ \\
\hline \multicolumn{2}{|c|}{ Average } & $8.46 \pm 0.91$ & $1.12 \pm 0.12$ \\
\hline
\end{tabular}

${ }^{\mathrm{a}}$ This single measurement was previously reported in ref. ${ }^{15}$.

Table S9 Crosslink Density of a3HBA Networks Determined through DMA

\begin{tabular}{|c|c|c|c|}
\hline Batch \# & Specimen \# & $\mathrm{E}_{\mathrm{r}}^{\prime}$ in rubbery plateau $(\mathrm{MPa})$ at $30^{\circ} \mathrm{C}$ & $v_{c}\left(\mathrm{x} 10^{-3} \mathrm{~mol} / \mathrm{cm}^{3}\right)$ \\
\hline \multirow{3}{*}{1} & 1 & 7.16 & 0.95 \\
\hline & 2 & 7.68 & 1.02 \\
\hline & 3 & 8.61 & 1.14 \\
\hline \multicolumn{2}{|c|}{ Average of batch 1} & $7.82 \pm 0.73$ & $1.03 \pm 0.10$ \\
\hline \multirow{3}{*}{2} & 1 & 8.80 & 1.16 \\
\hline & 2 & 8.11 & 1.07 \\
\hline & 3 & 8.03 & 1.06 \\
\hline \multicolumn{2}{|c|}{ Average of batch 2} & $8.31 \pm 0.42$ & $1.10 \pm 0.06$ \\
\hline \multirow{3}{*}{3} & 1 & 7.47 & 0.99 \\
\hline & 2 & 7.66 & 1.01 \\
\hline & 3 & 7.70 & 1.02 \\
\hline \multicolumn{2}{|c|}{ Average of batch 3} & $7.61 \pm 0.12$ & $1.01 \pm 0.02$ \\
\hline \multicolumn{2}{|c|}{ Average } & $7.91 \pm 0.73$ & $1.05 \pm 0.10$ \\
\hline
\end{tabular}


Table S10: Crosslink Density of a4HBA Networks Determined through DMA

\begin{tabular}{|c|c|c|c|}
\hline Batch \# & Specimen \# & $\mathrm{E}_{\mathrm{r}}^{\prime}$ in rubbery plateau $(\mathrm{MPa})$ at $30^{\circ} \mathrm{C}$ & $v_{c}\left(\mathrm{x} 10^{-3} \mathrm{~mol} / \mathrm{cm}^{3}\right)$ \\
\hline $1^{\mathrm{a}}$ & 1 & 11.6 & 1.53 \\
\hline \multirow{3}{*}{2} & 1 & 9.87 & 1.31 \\
\hline & 2 & 10.3 & 1.36 \\
\hline & 3 & 11.1 & 1.47 \\
\hline \multicolumn{2}{|c|}{ Average of batch 2} & $10.4 \pm 0.6$ & $1.38 \pm 0.09$ \\
\hline \multirow{3}{*}{3} & 1 & 10.4 & 1.37 \\
\hline & 2 & 10.4 & 1.37 \\
\hline & 3 & 11.4 & 1.51 \\
\hline \multicolumn{2}{|c|}{ Average of batch 3} & $10.7 \pm 0.6$ & $1.42 \pm 0.08$ \\
\hline \multirow{3}{*}{4} & 1 & 9.09 & 1.20 \\
\hline & 2 & 9.63 & 1.27 \\
\hline & 3 & 9.51 & 1.26 \\
\hline \multicolumn{2}{|c|}{ Average of batch 4} & $9.41 \pm 0.28$ & $1.24 \pm 0.04$ \\
\hline \multicolumn{2}{|c|}{ Average } & $10.5 \pm 0.9$ & $1.39 \pm 0.12$ \\
\hline
\end{tabular}

a This single measurement was previously reported in ref. ${ }^{15}$.

Table S11: Crosslink Density of aGenA Networks Determined through DMA

\begin{tabular}{|c|c|c|c|}
\hline Batch \# & Specimen \# & $\mathrm{E}_{\mathrm{r}}^{\prime}$ in rubbery plateau $(\mathrm{MPa})$ at $30^{\circ} \mathrm{C}$ & $v_{c}\left(\mathrm{x} 10^{-3} \mathrm{~mol} / \mathrm{cm}^{3}\right)$ \\
\hline 1 & 1 & 12.2 & 1.61 \\
\hline \multirow{3}{*}{2} & 1 & 11.9 & 1.57 \\
\hline & 2 & 11.7 & 1.55 \\
\hline & 3 & 11.6 & 1.54 \\
\hline \multicolumn{2}{|c|}{ Average of batch 2} & $11.7 \pm 0.1$ & $1.55 \pm 0.02$ \\
\hline \multirow{3}{*}{3} & 1 & 10.6 & 1.40 \\
\hline & 2 & 10.7 & 1.42 \\
\hline & 3 & 10.6 & 1.40 \\
\hline \multicolumn{2}{|c|}{ Average of batch 3} & $10.6 \pm 0.1$ & $1.40 \pm 0.01$ \\
\hline \multirow{3}{*}{4} & 1 & 10.3 & 1.36 \\
\hline & 2 & 10.4 & 1.37 \\
\hline & 3 & 10.6 & 1.40 \\
\hline \multicolumn{2}{|c|}{ Average of batch 4} & $10.4 \pm 0.1$ & $1.37 \pm 0.02$ \\
\hline \multicolumn{2}{|c|}{ Average } & $11.2 \pm 0.9$ & $1.49 \pm 0.12$ \\
\hline
\end{tabular}


Table S12: Crosslink Density of aGalA Networks Determined through DMA

\begin{tabular}{|c|c|c|c|}
\hline Batch \# & Specimen \# & $\mathrm{E}_{\mathrm{r}}^{\prime}$ in rubbery plateau $(\mathrm{MPa})$ at $30^{\circ} \mathrm{C}$ & $v_{c}\left(\times 10^{-3} \mathrm{~mol} / \mathrm{cm}^{3}\right)$ \\
\hline 1 & 1 & 13.3 & 1.75 \\
\hline \multirow{3}{*}{2} & $2-1$ & 10.8 & 1.43 \\
\hline & $2-2$ & 11.3 & 1.50 \\
\hline & $2-3$ & 12.0 & 1.59 \\
\hline \multicolumn{2}{|c|}{ Average of batch 2} & $11.4 \pm 0.6$ & $1.51 \pm 0.08$ \\
\hline \multirow{3}{*}{3} & $3-1$ & 10.3 & 1.36 \\
\hline & $3-2$ & 10.2 & 1.34 \\
\hline & $3-3$ & 9.7 & 1.28 \\
\hline \multicolumn{2}{|c|}{ Average of batch 3} & $10.0 \pm 0.3$ & $1.33 \pm 0.04$ \\
\hline \multirow{3}{*}{4} & $4-1$ & 9.9 & 1.31 \\
\hline & $4-2$ & 10.6 & 1.41 \\
\hline & 4-3 & 10.7 & 1.42 \\
\hline \multicolumn{2}{|c|}{ Average of batch 4} & $10.4 \pm 0.5$ & $1.38 \pm 0.06$ \\
\hline \multicolumn{2}{|c|}{ Average } & $11.3 \pm 1.4$ & $1.49 \pm 0.19$ \\
\hline
\end{tabular}


Table S13: $T_{g}$ of a3HBA Networks Determined through DSC

\begin{tabular}{|c|c|c|c|}
\hline Batch \# & Specimen \# & 1st heating scan & 2nd heating scan \\
\hline \multirow{9}{*}{1} & 1 & -5.1 & -4.9 \\
\hline & 2 & -5.6 & -5.5 \\
\hline & 3 & -4.7 & -4.5 \\
\hline & 4 & -6.3 & -6.2 \\
\hline & 5 & -6.9 & -6.9 \\
\hline & 6 & -5.5 & -5.4 \\
\hline & 7 & -3.0 & -3.0 \\
\hline & 8 & -3.5 & -3.5 \\
\hline & 9 & -3.2 & -3.1 \\
\hline \multicolumn{2}{|c|}{ Average of batch 1} & $-4.9 \pm 1.4$ & $-4.8 \pm 1.4$ \\
\hline \multirow{3}{*}{2} & 1 & -8.5 & -8.4 \\
\hline & 2 & -8.1 & -8.0 \\
\hline & 3 & -7.43 & -7.2 \\
\hline \multicolumn{2}{|c|}{ Average of batch 2} & $-8.0 \pm 0.5$ & $-7.9 \pm 0.6$ \\
\hline \multirow{3}{*}{3} & 1 & -8.5 & -8.6 \\
\hline & 2 & -8.5 & -8.2 \\
\hline & 3 & -8.1 & -7.9 \\
\hline \multicolumn{2}{|c|}{ Average of batch 3} & $-8.4 \pm 0.2$ & $-8.2 \pm 0.3$ \\
\hline \multicolumn{2}{|c|}{ Average } & $-7.1 \pm 1.9$ & $-6.9 \pm 1.9$ \\
\hline
\end{tabular}


Table S14: $T_{g}$ of aGenA Networks Determined through DSC

\begin{tabular}{|c|c|c|c|}
\hline Batch \# & Specimen \# & 1st heating scan & 2nd heating scan \\
\hline \multirow{9}{*}{1} & 1 & -4.4 & -4.2 \\
\hline & 2 & -6.4 & -6.3 \\
\hline & 3 & -5.3 & -5.0 \\
\hline & 4 & -5.0 & -4.6 \\
\hline & 5 & -7.5 & -7.6 \\
\hline & 6 & -8.1 & -8.1 \\
\hline & 7 & -5.1 & -5.2 \\
\hline & 8 & -7.4 & -7.4 \\
\hline & 9 & -7.9 & -7.9 \\
\hline \multicolumn{2}{|c|}{ Average of batch 1} & $-6.3 \pm 1.4$ & $-6.3 \pm 1.5$ \\
\hline \multirow{3}{*}{2} & 1 & -4.3 & -3.6 \\
\hline & 2 & -5.2 & -4.6 \\
\hline & 3 & -3.3 & -2.9 \\
\hline \multicolumn{2}{|c|}{ Average of batch 2} & $-4.2 \pm 1.0$ & $-3.7 \pm 0.9$ \\
\hline \multirow{3}{*}{3} & 1 & -2.9 & -2.8 \\
\hline & 2 & -5.9 & -5.6 \\
\hline & 3 & -5.6 & -5.5 \\
\hline \multicolumn{2}{|c|}{ Average of batch 3} & $-4.8 \pm 1.7$ & $-4.6 \pm 1.6$ \\
\hline \multirow{3}{*}{4} & 1 & -8.2 & -8.1 \\
\hline & 2 & -7.3 & -6.9 \\
\hline & 3 & -6.5 & -6.5 \\
\hline \multicolumn{2}{|c|}{ Average of batch 4} & $-7.3 \pm 0.8$ & $-7.2 \pm 0.8$ \\
\hline \multicolumn{2}{|c|}{ Average } & $-5.7 \pm 1.7$ & $-5.4 \pm 1.6$ \\
\hline
\end{tabular}


Table S15: $\mathrm{T}_{\mathrm{g}}$ of aGalA Networks Determined through DSC

\begin{tabular}{|c|c|c|c|}
\hline Batch \# & Specimen \# & 1st heating scan & 2nd heating scan \\
\hline \multirow{9}{*}{1} & 1 & -10.0 & -9.9 \\
\hline & 2 & -9.1 & -8.6 \\
\hline & 3 & -8.9 & -8.6 \\
\hline & 4 & -4.7 & -4.6 \\
\hline & 5 & -6.5 & -6.6 \\
\hline & 6 & -6.6 & -6.1 \\
\hline & 7 & -6.4 & -5.9 \\
\hline & 8 & -6.8 & -6.1 \\
\hline & 9 & -8.2 & -7.7 \\
\hline \multicolumn{2}{|c|}{ Average of batch 1} & $-7.5 \pm 1.7$ & $-7.1 \pm 1.7$ \\
\hline \multirow{3}{*}{2} & 1 & -7.4 & -6.9 \\
\hline & 2 & -11.2 & -10.9 \\
\hline & 3 & -5.5 & -5.1 \\
\hline \multicolumn{2}{|c|}{ Average of batch 2} & $-8.1 \pm 2.9$ & $-7.7 \pm 3.0$ \\
\hline \multirow{3}{*}{3} & 1 & -5.4 & -5.0 \\
\hline & 2 & -8.5 & -8.3 \\
\hline & 3 & -5.9 & -5.7 \\
\hline \multicolumn{2}{|c|}{ Average of batch 3} & $-6.6 \pm 1.6$ & $-6.3 \pm 1.7$ \\
\hline \multirow{3}{*}{4} & 1 & -7.5 & -7.4 \\
\hline & 2 & -8.4 & -8.4 \\
\hline & 3 & -7.0 & -7.0 \\
\hline \multicolumn{2}{|c|}{ Average of batch 4} & $-7.6 \pm 0.7$ & $-7.6 \pm 0.7$ \\
\hline \multicolumn{2}{|c|}{ Average } & $-7.4 \pm 2.9$ & $-7.2 \pm 3.0$ \\
\hline
\end{tabular}


Table S16: Thermal Properties of Thiol-Ene Networks Derived from Phenolic Acids ${ }^{\mathrm{a}}$

\begin{tabular}{ccc} 
Allyltaed Phenolic acid & $\mathrm{T}_{\mathrm{g}}\left({ }^{\circ} \mathrm{C}\right)^{\mathrm{b}}$ & Onset degradation temperature $\left({ }^{\circ} \mathrm{C}\right)^{\mathrm{c}}$ \\
\hline aSA & $0.8 \pm 2.1$ & 341.9 \\
a3HBA & $-7.1 \pm 1.9$ & 349.2 \\
a4HBA & $5.5 \pm 2.2$ & 343.0 \\
aGenA & $-5.7 \pm 1.7$ & 339.1 \\
aGalA & $-7.4 \pm 2.9$ & 337.1 \\
\hline
\end{tabular}

${ }^{a}$ Samples were prepared following the protocol in Table 1.

${ }^{\mathrm{b}}$ Determined from DSC. $\mathrm{T}_{\mathrm{g}}$ measurements were obtained from several distinct specimens of three separate batches for each network type. The measurement errors were determined as the standard deviation obtained from either measurements within the same batch, or batch-to-batch measurements (whichever determination of error was greater). The results presented in this table were obtained from the first heating scan; the full data sets are shown in Tables S13-S15 for a3HBA, aGenA and aGalA, and were previously reported in ref. ${ }^{15}$ for aSA and a4HBA.

${ }^{\mathrm{c}}$ Determined from TGA. Previously reported in ref. ${ }^{15}$ for aSA and a4HBA. 

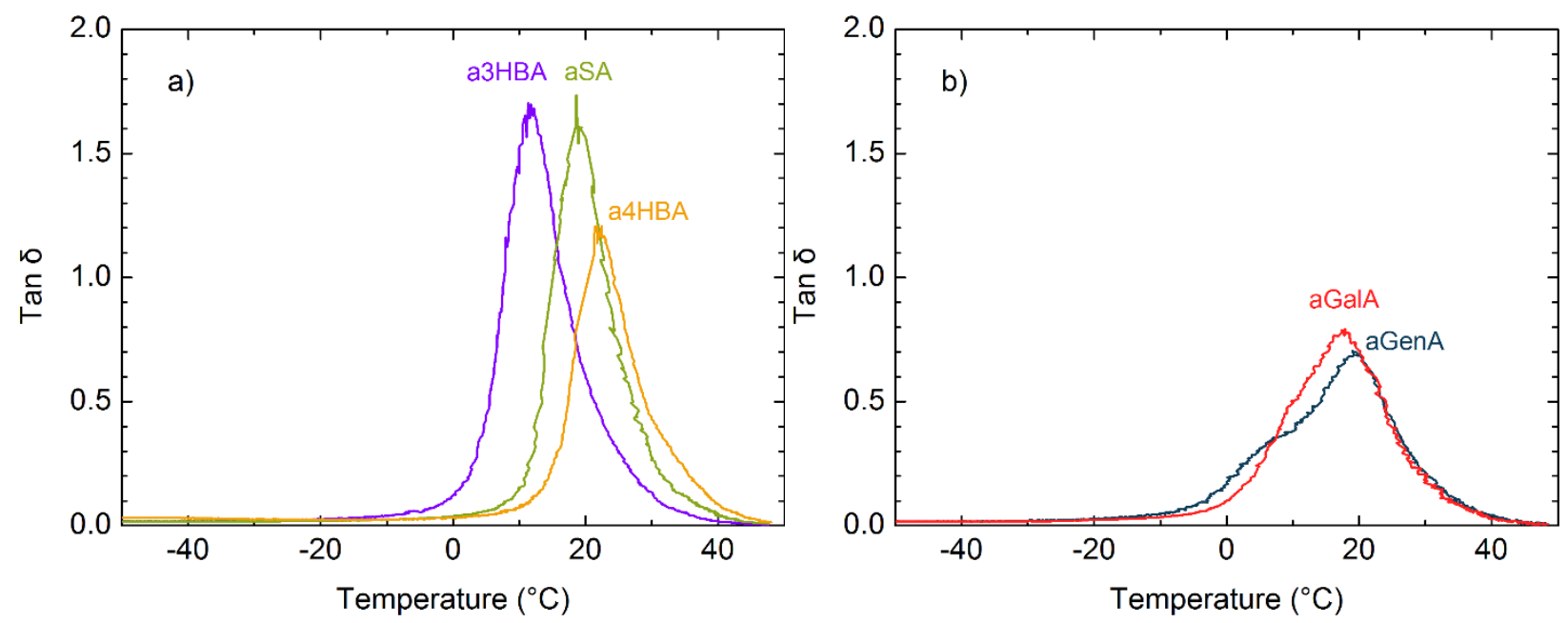

Figure S12: Tan $\delta\left(E^{\prime \prime} / E^{\prime}\right)$, measured through DMA, as a function of temperature of thiol-ene networks derived from a) aSA (green curve), a3HBA (purple curve), and a4HBA (yellow curve); and b) aGenA (blue curve) and aGalA (red curve). The temperature ramp was conducted at a rate of $3{ }^{\circ} \mathrm{C} / \mathrm{min}$, using a strain of $0.05 \%$ and frequency of $1 \mathrm{~Hz}$. 

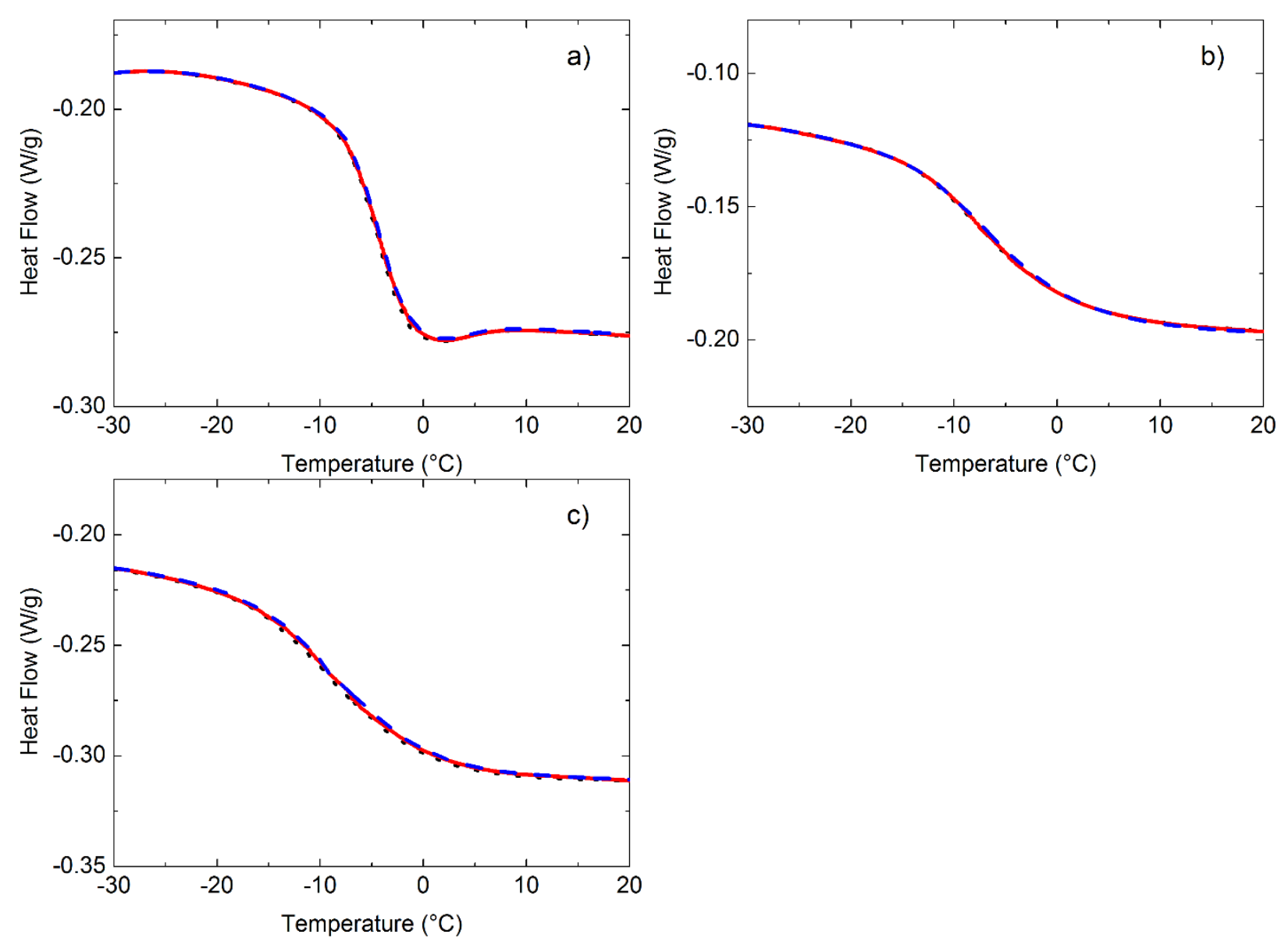

Figure S13: DSC heat flow as a function of temperature for thiol-ene networks derived from (a) a3HBA, (b) aGenA and (c) aGalA. Samples were exposed to UV for 15 min and were cured at $150{ }^{\circ} \mathrm{C}$ for $10 \mathrm{~min}$ (a3HBA) or $20 \mathrm{~min}$ (aGenA and aGalA), encapsulated into an aluminum pan, and subsequently cycled through three consecutive heating and cooling sequences in the DSC (at a rate of $10^{\circ} \mathrm{C} / \mathrm{min}$ ); the first heating scan is indicated by the black dotted curve, the second heating scan is indicated by the red solid curve, and the third heating scan is indicated by the blue dashed curve. 

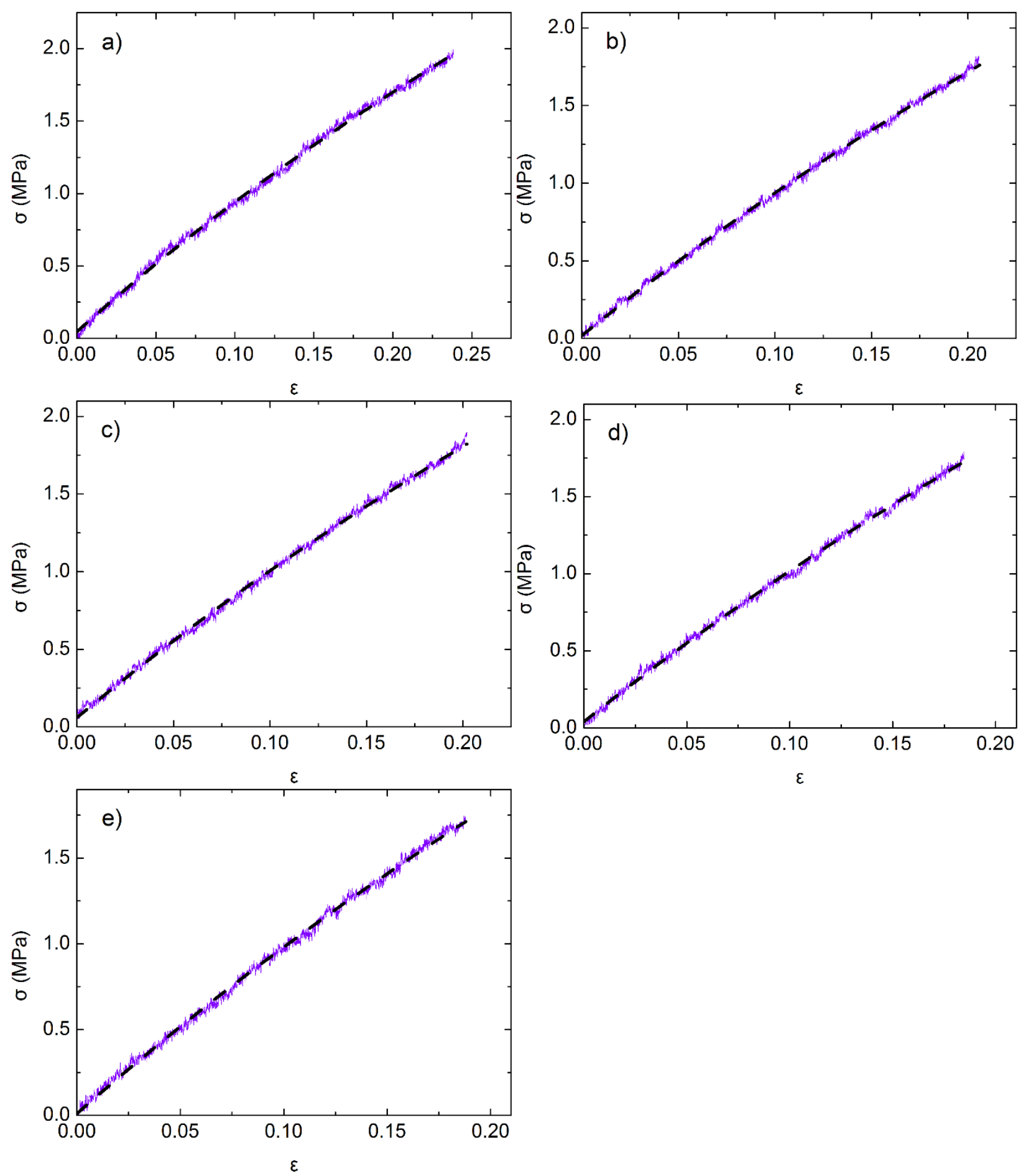

Figure S14: Stress-strain curves obtained from five independent specimens of thiol-ene networks derived from a3HBA (purple solid curve is the tensile data; black dashed curve shows the fit of the ideal elastomer model to the data). 

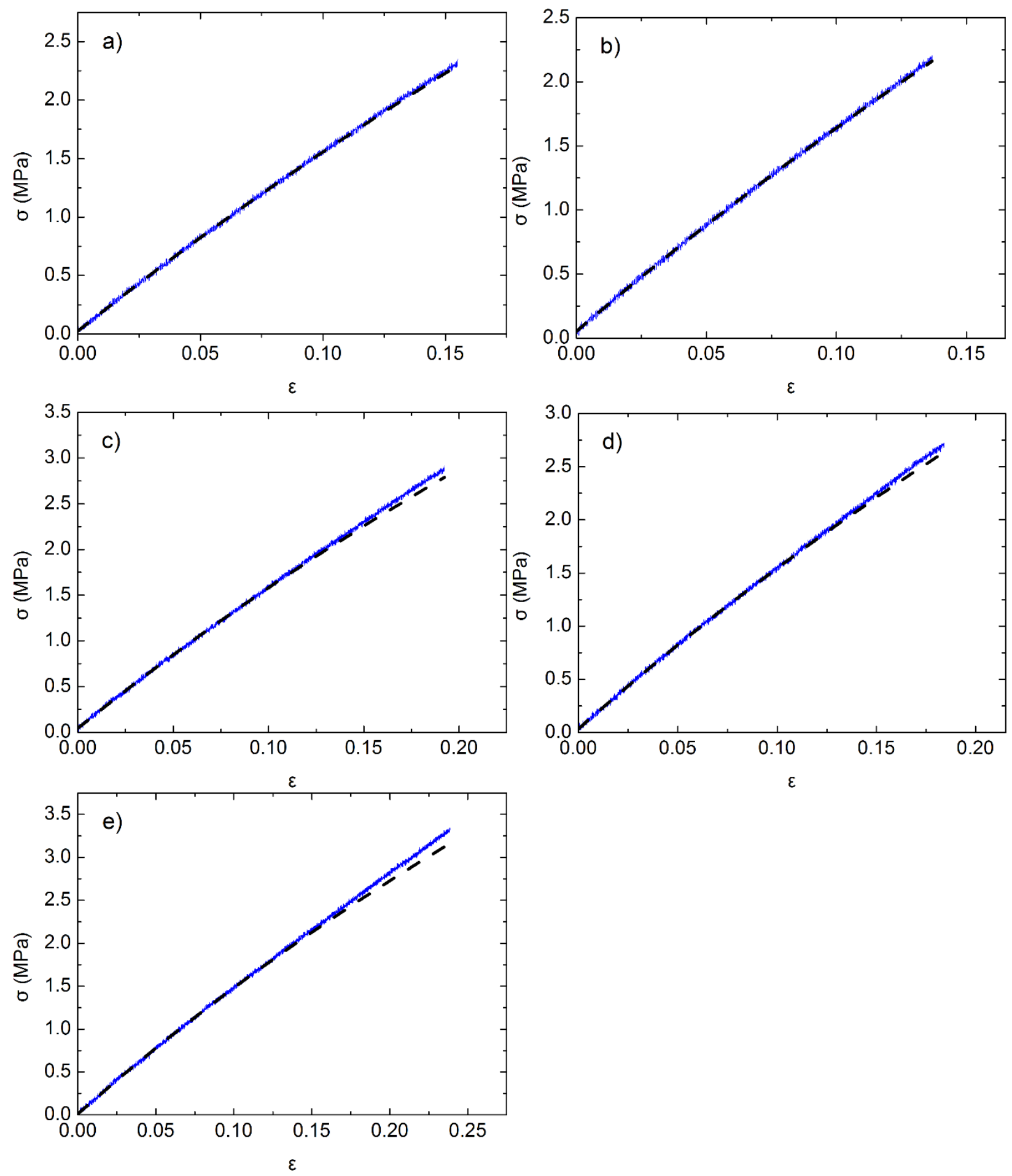

Figure S15: Stress-strain curves obtained from five independent specimens of thiol-ene networks derived from aGenA (blue solid curve is the tensile data; black dashed curve shows the fit of the ideal elastomer model to the data). 

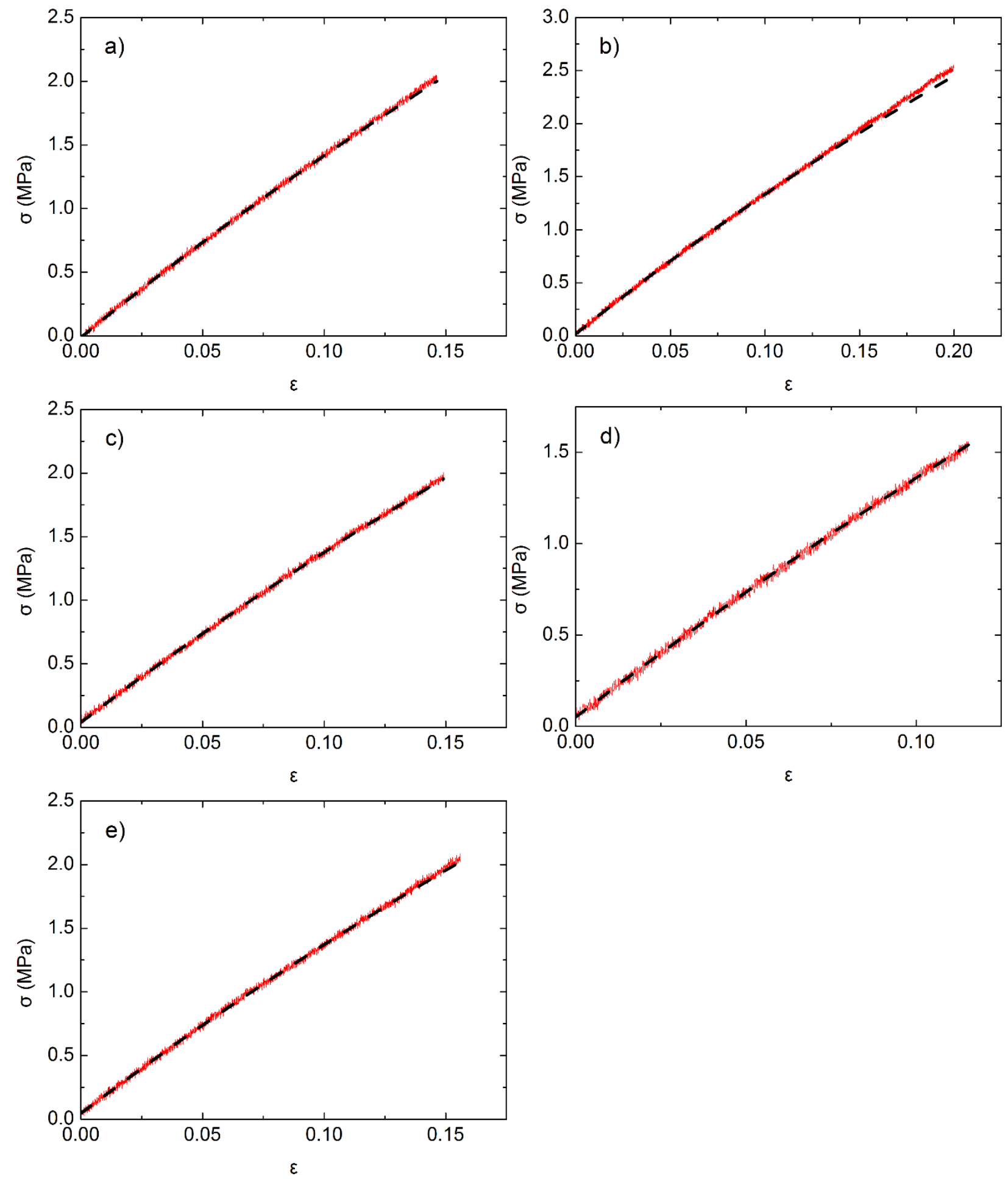

Figure S16: Stress-strain curves obtained from five independent specimens of thiol-ene networks derived from aGalA (red solid curve is the tensile data; black dashed curve shows the fit of the ideal elastomer model to the data). 
Table S17: Tensile Properties of Thiol-Ene Networks Derived from Allylated Phenolic Acids

\begin{tabular}{ccccc}
\hline $\begin{array}{c}\text { Allylated } \\
\text { Phenolic acid }\end{array}$ & $\begin{array}{c}\text { Tensile strength } \\
(\mathrm{MPa})\end{array}$ & $\begin{array}{c}\text { \% elongation at } \\
\text { break }\end{array}$ & $\begin{array}{c}\text { Modulus } \\
(\mathrm{MPa})\end{array}$ & $\begin{array}{c}\text { Toughness } \\
(\mathrm{MPa})\end{array}$ \\
\hline aSA $^{\mathrm{b}}$ & $2.7 \pm 0.3$ & $25.0 \pm 2.2$ & $10.8 \pm 0.4$ & $0.36 \pm 0.06$ \\
a3HBA & $1.8 \pm 0.1$ & $20.4 \pm 2.1$ & $8.6 \pm 0.5$ & $0.20 \pm 0.03$ \\
a4HBA $^{\mathrm{b}}$ & $3.7 \pm 0.3$ & $29.6 \pm 2.4$ & $12.4 \pm 0.3$ & $0.57 \pm 0.09$ \\
aGenA $_{\text {aGalA }}^{2.7 \pm 0.5}$ & $18.1 \pm 3.9$ & $14.6 \pm 0.6$ & $0.32 \pm 0.13$ \\
\hline
\end{tabular}

${ }^{\text {a Samples were prepared following the protocol in Table } 1 .}$

${ }^{\mathrm{b}}$ Previously reported in ref. ${ }^{15}$ for aSA and a4HBA.

Table S18: Parameters Extracted from the Fit of the Mooney-Rivlin Equation to the Tensile Data

\begin{tabular}{cccc}
\hline $\begin{array}{c}\text { Allylated } \\
\text { Phenolic acid }\end{array}$ & Strain Range & $\mathrm{C}_{1}$ & $\mathrm{C}_{2}$ \\
\hline a3HBA & All & $1.66 \pm 0.17$ & $0.12 \pm 0.18$ \\
aGenA $^{\mathrm{a}}$ & $\varepsilon<0.144 \pm 0.017$ & $2.60 \pm 0.35$ & $0.29 \pm 0.49$ \\
aGenA $^{\mathrm{a}}$ & $\varepsilon>0.144 \pm 0.017$ & $3.90 \pm 0.21$ & $-1.23 \pm 0.35$ \\
aGalA $^{\mathrm{b}}$ & $\varepsilon<0.153$ & $2.13 \pm 0.85$ & $0.43 \pm 0.91$ \\
aGalA $^{\mathrm{b}}$ & $\varepsilon>0.153$ & 3.45 & -1.14 \\
aSA $^{\mathrm{c}}$ & All & $2.20 \pm 0.21$ & $-0.07 \pm 0.32$ \\
a4HBA & $\varepsilon<0.202 \pm 0.008$ & $2.82 \pm 0.25$ & $-0.35 \pm 0.34$ \\
a4HBA & $\varepsilon>0.202 \pm 0.008$ & $4.10 \pm 0.16$ & $-1.88 \pm 0.18$ \\
\hline
\end{tabular}

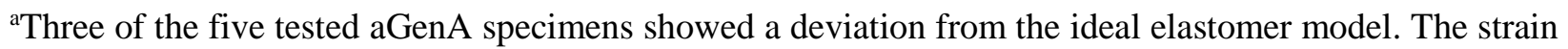
value at which the data deviated from the model differed from specimen to specimen (e.g. the deviation occurred at $\varepsilon=0.144 \pm 0.017$ for aGenA). The Mooney-Rivlin model was fit to the data both above and below this critical strain value.

${ }^{\mathrm{b}}$ One of the five tested specimens showed a deviation from the ideal elastomer model. The data deviated from the model $\varepsilon=0.153$. The Mooney-Rivlin model was fit to the data both above and below this critical strain value.

${ }^{\mathrm{c}}$ Mooney-Rivlin coefficient $\mathrm{C}_{2}$ for the aSA-based network was reported in ref. ${ }^{15}$. 
Table S19: Experimental Crosslink Density and Molecular Weight Between Crosslinks ${ }^{\mathrm{a}}$

\begin{tabular}{cccc}
\hline $\begin{array}{c}\text { Allylated } \\
\text { phenolic acid }\end{array}$ & $\begin{array}{c}\mathrm{E}^{\prime} \text { in rubbery plateau } \\
(\mathrm{MPa})^{\mathrm{b}}\end{array}$ & $v_{c}\left({\left.\mathrm{x} 10^{-3} \mathrm{~mol} / \mathrm{cm}^{3}\right)^{\mathrm{c}}}\right.$ & $\mathrm{M}_{\mathrm{c}}(\mathrm{g} / \mathrm{mol})^{\mathrm{d}}$ \\
\hline aSA & $8.46 \pm 0.91$ & $1.12 \pm 0.12$ & $1102 \pm 131$ \\
a3HBA & $7.91 \pm 0.73$ & $1.05 \pm 0.10$ & $1170 \pm 109$ \\
a4HBA & $10.5 \pm 0.9$ & $1.39 \pm 0.12$ & $882 \pm 77$ \\
aGenA & $11.2 \pm 0.9$ & $1.49 \pm 0.12$ & $825 \pm 64$ \\
aGalA & $11.3 \pm 1.4$ & $1.49 \pm 0.19$ & $828 \pm 99$ \\
\hline
\end{tabular}

${ }^{a}$ Samples were prepared following the protocol in Table 1.

${ }^{\mathrm{b}}$ Reduced storage modulus in rubbery plateau (DMA) at reference temperature $30^{\circ} \mathrm{C}(\omega=1 \mathrm{~Hz})$.

${ }^{\mathrm{c}} v_{\mathrm{c}}$ was calculated using Eqn. 6. All data and calculations are shown in Tables S5-S9

${ }^{d}$ Molecular weight between crosslinks $\left(M_{c}\right)$, was calculated using E' in the rubbery plateau $\left(M_{c}=\rho / v_{c}\right)$. The values of mass densities used in these calculations $(\rho)$ were measured for the thiol-ene networks and reported in the main text.

Table S20: Theoretical Crosslink Density and Molecular Weight Between Crosslinks

\begin{tabular}{|c|c|c|c|c|c|}
\hline & $\begin{array}{c}\text { Allylated } \\
\text { phenolic } \\
\text { acid }\end{array}$ & $\mathrm{M}_{\mathrm{c}}(\mathrm{g} / \mathrm{mol})^{\mathrm{a}}$ & $\begin{array}{l}v_{c}\left(\times 10^{-3}\right. \\
\left.\mathrm{mol} / \mathrm{cm}^{3}\right)^{\mathrm{c}}\end{array}$ & $\begin{array}{c}\mathrm{M}_{\mathrm{c}}(\mathrm{g} / \mathrm{mol}) \\
\text { average }^{\mathrm{b}}\end{array}$ & $\begin{array}{c}v_{c}\left({\mathrm{x} 10^{-3}}^{-3}\right. \\
\left.\mathrm{mol} / \mathrm{cm}^{3}\right) \\
\text { average }^{\mathrm{c}}\end{array}$ \\
\hline Assuming & aSA & 456 & 2.68 & 456 & 2.68 \\
\hline $100 \%$ & a3HBA & 456 & 2.68 & 456 & 2.68 \\
\hline Conversion of & a4HBA & 456 & 2.68 & 456 & 2.68 \\
\hline Functional & aGenA & $176 / 204$ & $6.93 / 5.98$ & 185 & 6.61 \\
\hline Groups & aGalA & $176 / 204$ & $6.93 / 5.98$ & 183 & 6.69 \\
\hline Accounting & aSA & 456 & 2.68 & 456 & 2.68 \\
\hline for Measured & a3HBA & 456 & 2.68 & 456 & 2.68 \\
\hline Conversion of & a4HBA & 456 & 2.68 & 456 & 2.68 \\
\hline Functional & $\mathrm{aGen} A$ & $176 / 204 / 512$ & $6.93 / 5.98 / 2.38$ & 225 & 6.11 \\
\hline Groups $^{\mathrm{d}}$ & aGalA & $176 / 204$ & $6.93 / 5.98$ & 183 & 6.69 \\
\hline
\end{tabular}

${ }^{a}$ The theoretical $\mathrm{M}_{\mathrm{c}}$ was calculated considering a perfect network (without any defects) and using the chemical structures of the allylated phenolic acids and multifunctional thiol. Details are shown below in Figure S17.

${ }^{\mathrm{b}}$ In the case of multiple values of $\mathrm{M}_{\mathrm{c}}$ that are calculated theoretically, an average is reported here. $100 \%$ conversion:

For GenA: Average $\mathrm{M}_{\mathrm{c}}=(1 / 3)(204)+(2 / 3)(176)=185 \mathrm{~g} / \mathrm{mol}$

For GalA: Average $\mathrm{M}_{\mathrm{c}}=(1 / 4)(204)+(3 / 4)(176)=183 \mathrm{~g} / \mathrm{mol}$

Accounting for measured reaction conversion (more details included below Figure S17):

For GenA (88\% conversion): Average $\mathrm{M}_{\mathrm{c}}=(0.88)(1 / 3)(204)+(0.88)(2 / 3)(176)+(0.12)(512)=225 \mathrm{~g} / \mathrm{mol}$

${ }^{c} v_{c}$ is calculated from the theoretical $M_{c}\left(v_{c}=\rho / M_{c}\right)$. The values of mass densities used in these calculations $(\rho)$ were measured for the thiol-ene networks and reported in the main text.

${ }^{\mathrm{d}}$ The experimentally measured conversions for each network type were considered in these calculations. More details are provided below Figure S17. 
Figure S17: Theoretical $\mathrm{M}_{\mathrm{c}}$ Calculations:<smiles>O=C(CCS)OCC(COC(=O)CCS)(COC(=O)CCS)COC(=O)CCS</smiles>
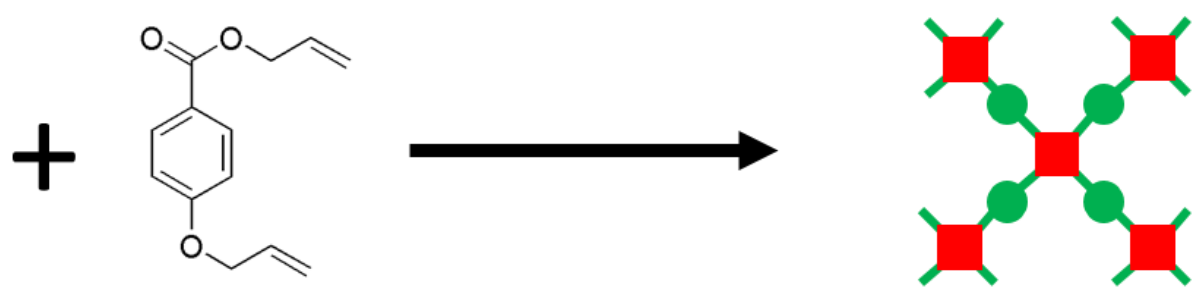

Figure S17i: Photoinitated thiol-ene reaction between a4HBA and the tetra-functional thiol PETMP. The other difunctional allylated phenolic acids (aSA, a3HBA) undergo comparable reactions.

a)

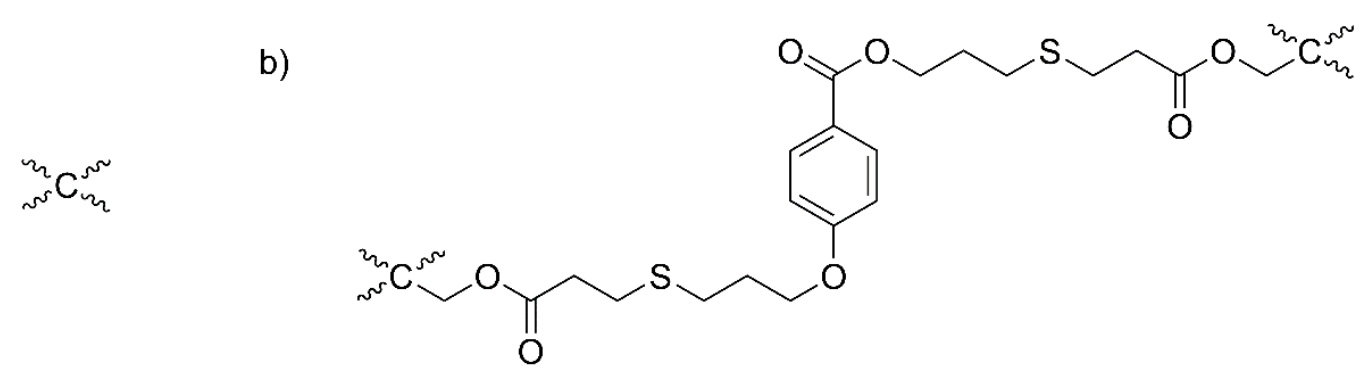

Figure S17ii: Chemical structures of a) junctions and b) strands in a4HBA thiol-ene networks. The molecular weight of a strand is $456 \mathrm{~g} / \mathrm{mol}$.
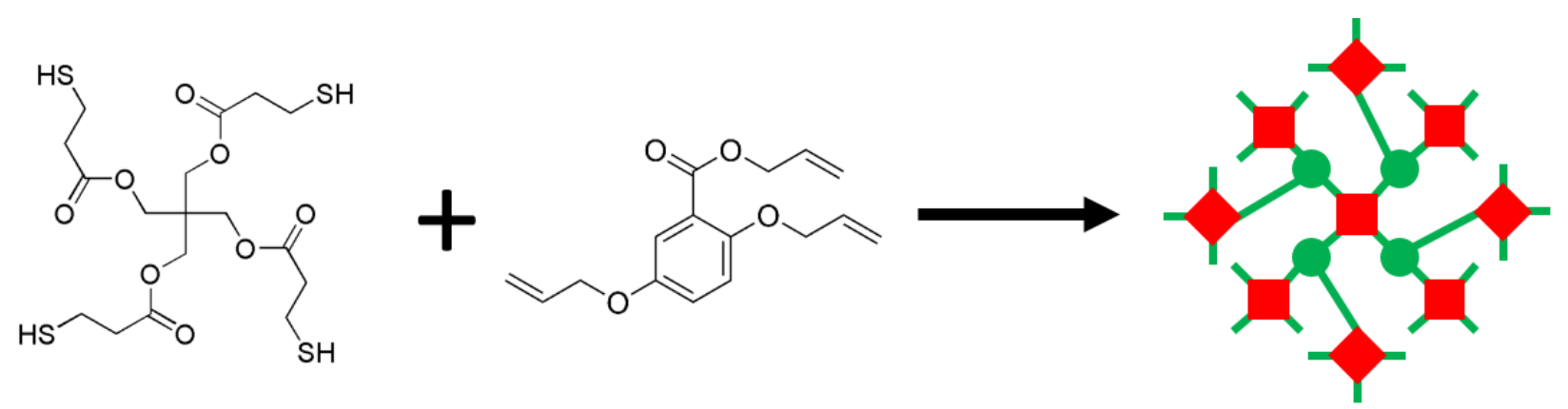

Figure S17iii: Photoinitated thiol-ene reaction between aGenA and the tetra-functional thiol PETMP. 
a)

$$
{ }_{n}^{n} C_{n}^{n}
$$

b)<smiles>Cc1ccc(C)c(C)c1</smiles>

c)<smiles>Cc1ccc(OCCCSCCC(=O)OCC(C)(C)C)cc1C</smiles>

d)<smiles>Cc1ccc(C)c(C(=O)OCCCSCCC(=O)OCC(C)(C)C)c1</smiles>

Figure S17iv: Chemical structures of a) b) junctions and c) d) strands in aGenA thiol-ene networks. The molecular weights of strands are 176 (ether) or 204 (ester) g/mol.
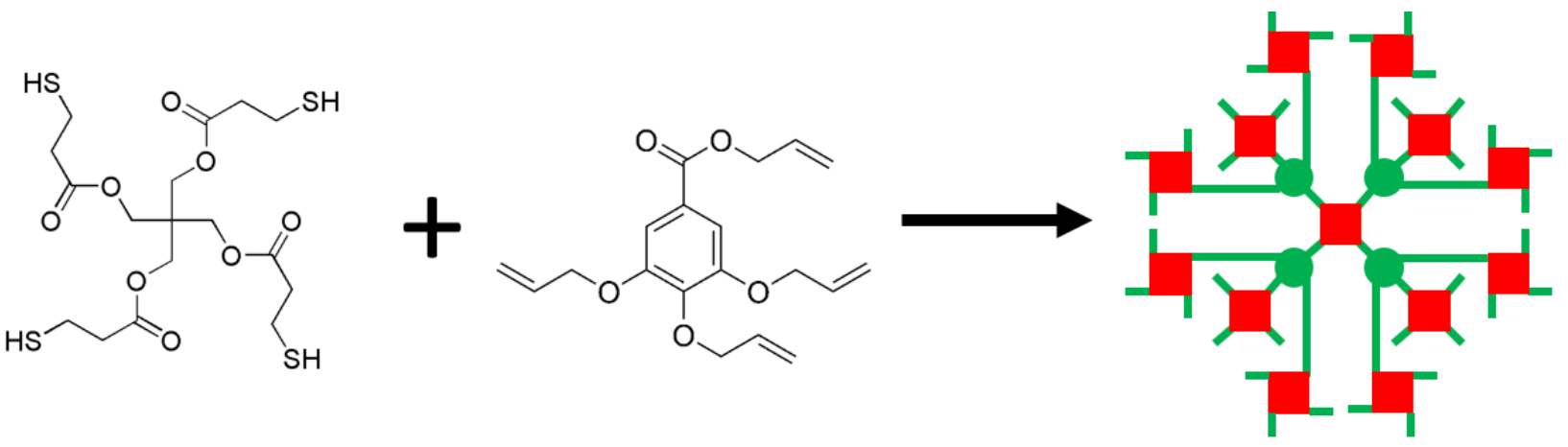

Figure S17v: Photoinitated thiol-ene reaction between aGalA and the tetra-functional thiol PETMP. 
a)

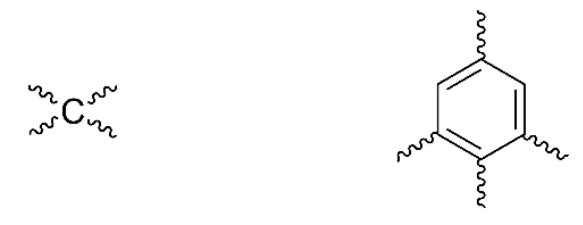

c)<smiles>Cc1cc(C)c(C)c(OCCCSCCC(=O)OCC(C)(C)C)c1</smiles>

d)<smiles>Cc1cc(C(=O)OCCCSCCC(=O)OCC(C)(C)C)cc(C)c1C</smiles>

Figure S17vi: Chemical structures of a) b) junctions and c) d) strands in aGalA thiol-ene networks. The molecular weights of strands are 176 (ether) or 204 (ester) g/mol.

a)<smiles>C=CCOC(=O)c1cc(OCCCSCCC(=O)OCC(C)(C)C)ccc1OCCCSCCC(=O)OCC(C)(C)C</smiles>

b)

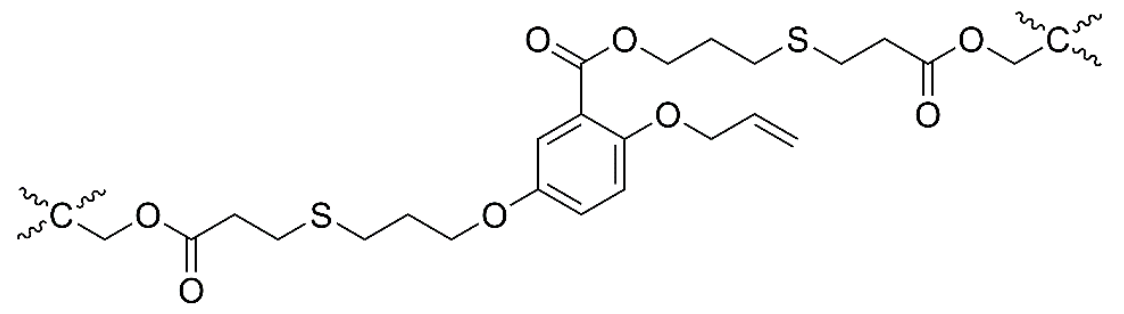

Figure S17vii: Chemical structures of strands in aGenA thiol-ene networks which are not fully reacted (i.e. one unreacted allyl group). The molecular weight of the strand is now $512 \mathrm{~g} / \mathrm{mol}$. 


\section{Calculation of theoretical crosslink density and $M_{c}$, accounting for experimentally measured conversions:}

The trends observed in the crosslink density do not correlate with trends in reaction conversion. For example, the aSA network had the highest reaction conversion (97\%), yet it had the second lowest crosslink density of all of the networks (Table S19). Furthermore, the a3HBA, a4HBA, and aGenA all had the same reaction conversion (88\%), yet very different crosslink densities. The aGalA network, with the lowest reaction conversion (82\%), still had a high crosslink density (similar to that of the a4HBA and aGenA networks). We therefore believe that differences in crosslink densities are due to differences in network architecture and not differences in reaction conversion. We have made the calculations of theoretical $\mathrm{M}_{\mathrm{c}}$ and $v_{\mathrm{c}}$ accounting for differences in reaction conversion (which were measured through FTIR). These results are also summarized in the bottom half of Table S20. Accounting for the reaction conversion has little impact on these trends:

- In the difunfunctional allylated phenolic acids (aSA, a4HBA, a3HBA), the network architecture is most heavily impacted by the thiol molecule functionality, which in our case is tetrafunctional. If we make the assumption that there is one unreacted functional group on a thiol molecule (or similarly, if there is an attached ene molecule that did not react on its other end), it is still a trifunctional network junction. This will therefore not impact the calculated theoretical molecular weight between crosslinks (see Figure S17). However, if there are two unreacted thiol molecules (or similarly, two ene molecules that reacted with one thiol but failed to react with a second thiol), then this thiol molecule will no longer be a junction and that strand will have double the value of $\mathrm{M}_{\mathrm{c}}$. However, it is most likely that the majority of unreacted functional groups will be found on differing thiols (as our conversions are relatively high: $97 \%$ for the aSA network and $88 \%$ for the a3HBA and a4HBA networks), and therefore to a first approximation, we do not think that the crosslink density in the aSA, a3HBA, and a4HBA networks will be significantly impacted by the unreacted functional groups.

- In the aGenA network, in which aGenA is a trifunctional molecule, there is a more important effect of reaction conversion. Both the thiol and allyl molecules act as junctions in this network (as the allyl molecules also have functionality greater than 2). We made the assumption that there could be one unreacted functional group on a aGenA molecule, shown in Figure S17vii. In this case, the strand length would increase from around 200 $\mathrm{g} / \mathrm{mol}$ to $512 \mathrm{~g} / \mathrm{mol}$. As the conversion in the aGenA network was $88 \%$, this would slightly increase the overall $\mathrm{M}_{\mathrm{c}}$ and decrease the crosslink density (Table S20).

- In the aGalA network, the conversion was the lowest of all of the networks (82\%). However, if there is one unreacted allyl group on a aGalA molecule, it will still be trifunctional, in which case it still will act as a network junction. If there are two unreacted allyl groups on a aGalA molecule, then it will no longer be a junction and in that case the crosslink desnity would decrease. However, to a first approximate, if we assume that there is only one unreacted allyl group on any given aGalA molecule, then there will be negligible impact on the crosslink density. 

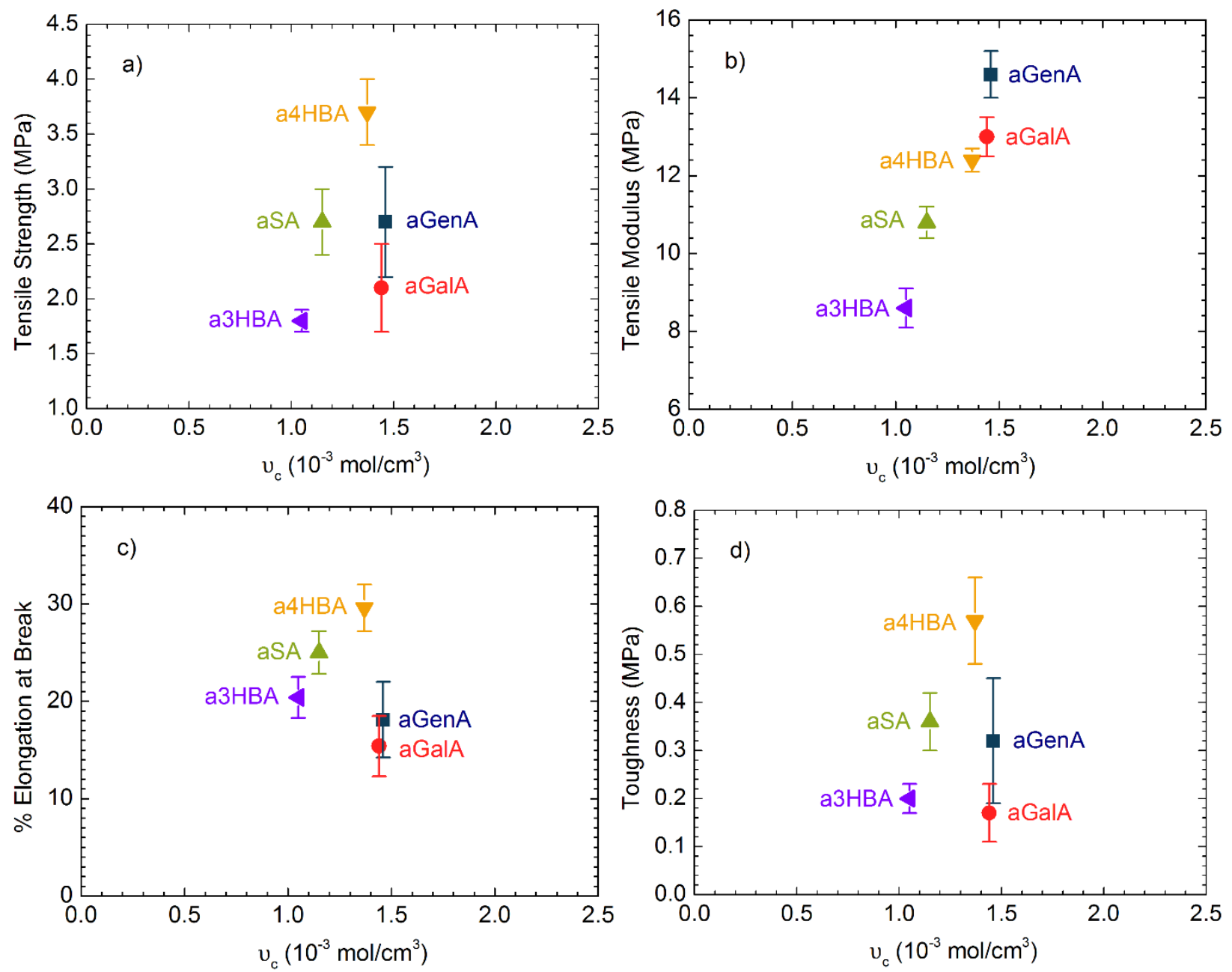

Figure S18: (a) Tensile strength, (b) tensile modulus, (c) \% elongation at break and (d) tensile toughness for thiol-ene networks derived from aSA (green $\boldsymbol{\Delta}$ ), a3HBA (purple $\triangleleft$ ), a4HBA (yellow $\boldsymbol{\nabla}$ ), aGenA (dark blue $\mathbf{m}$ ) and aGalA (red $\bullet$ ) as functions of the crosslink density ( $v_{c}$ ) measured from DMA. The standard deviations on these plots indicate error characterized through multiple measurements obtained on multiple independently prepared specimens (Table S16). Data obtained on aSA and a4HBA networks were previously reported in ref. ${ }^{15}$. 


\section{References}

1. Yang, Z.; Wicks, D. A.; Hoyle, C. E.; Pu, H.; Yuan, J.; Wan, D.; Liu, Y. Newly UV-curable polyurethane coatings prepared by multifunctional thiol- and ene-terminated polyurethane aqueous dispersions mixtures: Preparation and characterization. Polymer 2009, 50 (7), 1717-1722.

2. $\quad$ Yang, Z.; Wicks, D. A.; Yuan, J.; Pu, H.; Liu, Y. Newly UV-curable polyurethane coatings prepared by multifunctional thiol- and ene-terminated polyurethane aqueous dispersions: Photopolymerization properties. Polymer 2010, 51 (7), 1572-1577.

3. Ortiz, R. A.; Flores, R. V. G.; García Valdéz, A. E.; Duarte, M. L. B. Novel second generation dendrimer with terminal thiol groups and its thiol-ene photopolymerization with unsaturated monomers. Progress in Organic Coatings 2010, 69 (4), 463-469.

4. Claudino, M.; Johansson, M.; Jonsson, M. Thiol-ene coupling of 1,2-disubstituted alkene monomers: The kinetic effect of cis/trans-isomer structures. European Polymer Journal 2010, 46 (12), 2321-2332.

5. Claudino, M.; van der Meulen, I.; Trey, S.; Jonsson, M.; Heise, A.; Johansson, M. Photoinduced thiol-ene crosslinking of globalide/ $\varepsilon$-caprolactone copolymers: Curing performance and resulting thermoset properties. Journal of Polymer Science Part A: Polymer Chemistry 2012, 50 (1), 16-24. 6. $\quad$ Silverstein, R. M.; Webster, F. X.; Kiemle, D., Spectrometric Identification of Organic Compounds. 7th Edition ed.; John Wiley \& Sons Hoboken, NJ, 2005.

7. $\quad$ Çakmakçı, E.; Mülazim, Y.; Kahraman, M. V.; Apohan, N. K. Flame retardant thiol-ene photocured coatings. Reactive and Functional Polymers 2011, 71 (1), 36-41.

8. Narayanan, J.; Jungman, M. J.; Patton, D. L. Hybrid dual-cure polymer networks via sequential thiol-ene photopolymerization and thermal ring-opening polymerization of benzoxazines. Reactive and Functional Polymers 2012, 72 (11), 799-806.

9. Zhou, J.; Zhang, Q.-y.; Chen, S.-j.; Zhang, H.-p.; Ma, A.-j.; Ma, M.-1.; Liu, Q.; Tan, J.-j. Influence of thiol and ene functionalities on thiol-ene networks: Photopolymerization, physical, mechanical, and optical properties. Polymer Testing 2013, 32 (3), 608-616.

10. Beyazkilic, Z.; Kahveci, M. U.; Aydogan, B.; Kiskan, B.; Yagci, Y. Synthesis of polybenzoxazine precursors using thiols: Simultaneous thiol-ene and ring-opening reactions. Journal of Polymer Science Part A: Polymer Chemistry 2012, 50 (19), 4029-4036.

11. Chan, J. W.; Yu, B.; Hoyle, C. E.; Lowe, A. B. The nucleophilic, phosphine-catalyzed thiol-ene click reaction and convergent star synthesis with RAFT-prepared homopolymers. Polymer 2009, 50 (14), 3158-3168.

12. Black, M.; Rawlins, J. W. Thiol-ene UV-curable coatings using vegetable oil macromonomers. European Polymer Journal 2009, 45 (5), 1433-1441.

13. Çakmakçı, E.; Mülazim, Y.; Kahraman, M. V.; Apohan, N. K. Preparation and characterization of boron containing thiol-ene photocured hybrid coatings. Progress in Organic Coatings 2012, 75 (1-2), 2832.

14. Yang, H.; Zhang, Q.; Lin, B.; Fu, G.; Zhang, X.; Guo, L. Thermo-sensitive electrospun fibers prepared by a sequential thiol-ene click chemistry approach. Journal of Polymer Science Part A: Polymer Chemistry 2012, 50 (20), 4182-4190.

15. Yang, G.; Kristufek, S. L.; Link, L. A.; Wooley, K. L.; Robertson, M. L. Synthesis and Physical Properties of Thiol-Ene Networks Utilizing Plant-Derived Phenolic Acids. Macromolecules 2015, 48 (23), 8418-8427. 